\title{
Characterization of the types of the Neotropical Pseudisobrachium (Hymenoptera: Bethylidae), with a key to species
}

\author{
Celso O. Azevedo \\ Departamento de Biologia, Universidade Federal do Espírito Santo. Avenida Marechal Campos 1468, Maruípe, \\ 29040-090 Vitória, Espírito Santo, Brasil. E-mail: bethylidae@gmail.com
}

\begin{abstract}
The identity of many Neotropical species of Pseudisobrachium Kieffer, 1904 is currently unreachable, mostly because the available descriptions and illustrations are not sufficiently accurate to allow precise identifications. To help solve the problem, 115 valid species and their junior synonyms were examined. Twelve nomenclatural acts are made, and 110 valid species are recognized for the Neotropical region. Two lectotypes are designated: Pristocera crassicornis Westwood and Pristocera haemorrhoidalis Westwood. Seven new species-group synonyms are proposed: Pseudisobrachium retusum Evans syn. nov. for P. pauxillum Evans; P. cunco Perez syn. nov. for P. erythrocephalum Evans; P. navajo Evans, P. rectangulatum Evans, P. emarginatum Evans and P. foutsi Evans syn. nov. for P. flavinervis Fouts; P. acuminatum Waichert \& Azevedo syn. nov. for P. Iatum Waichert \& Azevedo. The following new genus-group synonym is established: Parisobrachium Kieffer syn. nov. for Dissomphalus Ashmead. The following new combination and reinstated name are proposed: Dissomphalus albipes (Kieffer) comb. nov. and nom. rev. from Pseudisobrachium paraguayense Kieffer.
\end{abstract}

KEY WORDS. Dissomphalus; Parisobrachium; taxonomy; systematics.

RESUMO. Caracterização dos tipos Pseudisobrachium (Hymenoptera: Bethylidae) neotropicais, com uma chave de espécies. Atualmente é difícil reconhecer a identidade de muitas espécies neotropicais de Pseudisobrachium Kieffer, 1904, principalmente por que as descrições e ilustrações disponíveis não são suficientes para permitir identificações precisas. Para resolver este problema, foram examinadas 115 espécies válidas, além de seus sinônimos juniores. Foram realizados doze atos nomenclaturais, e reconhecidas 110 espécies válidas para a região Neotropical. Foram designados dois lectótipos: Pristocera crassicornis Westwood and Pristocera haemorrhoidalis Westwood. Foram propostas sete sinonímias novas para espécies: Pseudisobrachium retusum Evans syn. nov. de P. pauxillum Evans; $P$. cunco Perez syn. nov. de $P$. erythrocephalum Evans; $P$. navajo Evans, $P$. rectangulatum Evans, $P$. emarginatum Evans e $P$. foutsi Evans syn. nov. de P. flavinervis Fouts; P. acuminatum Waichert \& Azevedo syn. nov. de P. Iatum Waichert $8 X$ Azevedo. Foi proposta a seguinte sinonímia nova para gênero: Parisobrachium Kieffer syn. nov. de Dissomphalus Ashmead. Foi estabelecida a seguinte combinação nova e revalidado o nome: Dissomphalus albipes (Kieffer) comb. nov. e nom. rev. de Pseudisobrachium paraguayense Kieffer.

PALAVRAS-CHAVE. Dissomphalus; Parisobrachium; taxonomia; sistemática.

The known world fauna of Pseudisobrachium Kieffer, 1904 is currently represented by 164 valid species, of which 115 are recorded from the Neotropical region, or $71 \%$ of the world fauna. It is not exaggeration to say, however, that these figures are almost meaningless, since there are hundreds of undescribed species in museums throughout the world (personal observation). Currently, Pseudisobrachium is the second largest genus in the Neotropical region. It is widespread in this area, and one of the most frequently captured bethylid genus, regardless of trap type or vegetation (e.g. Azevedo 1991, 1996, Azevedo \& Helmer 1999, Azevedo et al. 2002, 2006).

There are no specific studies on the life style of any Pseudisobrachium species. AsHMEAD (1893) found some Pseudisobrachium associated with formicid and coleopterous larvae (Evans 1964), but there is still no confirmation that these larvae are used as hosts.

Westwood (1874) described the three first Neotropical species of Pseudisobrachium originally in Pristocera Klug. CAMERON (1888, 1909), AsHMEAd (1894), KiefFer (1904, 1906, 1910a, b), MANN (1915), BRUCH (1917a, b) and Fouts (1928) proposed another 18 species. The corresponding descriptions, however, are all very superficial, insufficient to allow precise identifications. OGLoblin (1925a, b, 1938, 1950, 1963) described nine species, mostly based on females. I was not able to find the respective holotypes, except for one, but some of Ogloblin's descriptions are quite detailed, allowing precise identifications. Evans (1961, 1964, 1966, 1967, 1969a, b, c, 1973) proposed another 73 species, nearly $61 \%$ of the known Neotropical species at the time. Most of Evans' 
descriptions, however, had no illustrations at all, and characters of external genitalia were also not described for nearly all species, making it hard or impossible to determine the identity of such species. Although the external genitalia in Pseudisobrachium are not as diverse as in most of genera of Bethylidae, they can be useful in differentiating closely related species sometimes. Perez (1981), Vargas \& Terayama (2002), and Waichert \& AzeVedo (2004), described and illustrated 17 species, all of them fully recognizable through the respective descriptions alone.

The identity of most Neotropical species of Pseudisobrachium is therefore currently unreachable, mostly because the available descriptions and illustrations are not sufficiently accurate to allow precise identifications. Because of that, there are incorrect identifications for species of this genus in some museums (personal observation).

There is also no key to the Neotropical species of Pseudisobrachium. Actually, the only available key is to the North and Central America species, in Evans (1961), but it does not work with the Neotropical species.

The alpha-taxonomic limit and phylogenetic definition of Pseudisobrachium remains ill-defined, as with most bethylid genera. Because of this, some keys fail to identify precisely characterize the genus. The best way to recognize Pseudisobrachium is by examining male genitalia characters, such as the paramere complete and divided into two distinct arms, and the presence of a large grooved lobe in the inner margin of basivolsella, a feature named vannus by Evans (1961).

Evans (1961) split Pseudisobrachium species from North and Central America into six groups, but they simply do not work for Neotropical species, so I do not follow them here.

The male/female association represents yet another problem for the study of Pseudisobrachium. Of the 115 known Neotropical species, male and female have been associated for only one of them, Pseudisobrachium obscurum Evans. This happens because sexual dimorphism is extreme, making sex associations a difficult task. Males are large, winged and abundantly sculptured, while females are the only sex without ocelli, notauli, parapsidal furrows, wings, and show few carinae and grooves. Evans (1961) proposed sex association for Pseudisobrachium obscurum tentatively, without any explanation on how this was achieved. Presumably, he did this simply because he found the five females in South-eastern Arizona, where many males, including the holotype, are known to occur.

The main goal of this study is to organize the taxonomic knowledge of the Neotropical species of Pseudisobrachium, based mostly on the review of primary type material for all the available species names of the genus.

\section{MATERIAL AND METHODS}

\section{Specimens}

Efforts were made to study every holotype and allotype specimens of all the 115 valid Neotropical species, as well as type specimens of three junior synonymies. Types of 118 nomi- nal species were examined, but the type material of eight species could not be found or studied.

\section{Collections}

The examined material was kindly provided by the following institutions and curators: (ACC) Instituto de Ecología y Sistemática de Cuba (R. Núñez A.); (AMNH) American Museum of Natural History, USA (J. M. Carpenter); (BMNH) Natural History Museum, United Kingdom (K. Goodger); (CASC) California Academy of Sciences, USA (R. Zuparko); (CNCI) Canadian National Collection of Insects, Canada (J. T. Huber); (CUIC) Cornell University, USA (E. R. Hoebecke); (FIML) Fundación y Instituto Miguel Lillo, Argentina (V. Colomo); (IAVH) Instituto Alexander von Humboldt, Colombia (M. Ospina); (MACN) Museo Argentino de Ciencias Naturales Bernardino Rivadavia, Argentina (A. Roig Alsina); (MCSN) Museo Civico di Storia Naturale Giacomo Doria, Italy (R. Poggy); (MCZH) Museum of Comparative Zoology, USA (S.Cover \& P.D.Perkins); (NHME) Natuurhistorisch Museum Maastricht, Holand (F.H. DingemansBakels); (OXUM) Oxford University Museum of Natural History (C. O'Toole and J. E. Hogan); (SEMC) Snow Entomological Collection, The University of Kansas, USA (Z. Falin); (UFES) Universidade Federal do Espírito Santo, Brazil (author); (USNM) National Museum of Natural History, USA (T. Schultz, D. Furth); (ZMHB) Zoologisches Museum an der Humboldt-Universität zu Berlin, Germany (F. Koch).

Types of Pseudisobrachium deposited in the following institutions, according to the literature, could not be located: (MEUC) Universidad Metropolitana de Ciencias de la Educación, Chile (Jaime Solervicens); (MLPA) Museo de la Plata, Universidad de la Plata, Argentina (A. H. Abrahamovich); (NMPC) Narodni Muzeum Praha, Czech Republic (Jan Macek); Ogloblin Private Collection (Argentina); Perez Private Collection (Chile).

\section{Taxonomic Treatment}

The descriptions and illustrations of the material were prepared considering only the most important and useful characters in separating Pseudisobrachium species.

The taxonomic and synonymic list for the species are not presented; these can be found in GORDH \& Móczár (1990), which are still updated for Pseudisobrachium, except only by new species descriptions, namely two by Vargas \& Terayama (2002) and 14 by Waichert \& AzEvedo (2004).

Measurements and indexes used in this study are as follows. Body length, measured from the apex of clypeus to the posterior margin of the last metasomal segment, excluding male genitalia or female sting; (LH) length of head, measured in frontal view, from vertex crest to median apical margin of clypeus; (WH) width of head, measured in frontal view, its maximum width including eyes; (WF) minimum width of frons, measured in frontal view; the minimum width is usually attained near the level of the ventral margin of the eyes; (HE) maximum height of eye, measured in lateral view; (OOL) ocelli-ocular line, 
measured in latero-dorsal view, the shortest distance from eye top to posterior ocellus; (WOT) maximum width of ocellar triangle, including ocelli, measured in frontal view; (DAO) diameter of anterior ocellus, measured in frontal view; Distance of ocellar triangle to vertex: distance from posterior ocellus to vertex crest, measured in dorso-posterior view; (VOL) vertexocular line, equivalent to the distance from eye top to vertex crest, measured in dorsal view.

The nomenclature of integument sculpture follows HARRIS (1979), and general terms follow Evans (1961, 1964), but few terms need clarification. Temple is the upper part of gena (RICHARDS 1977). As the head of Bethylidae is prognathous, the temple is the part between posterior margin of eye and vertex crest. It therefore corresponds to the posterior part of head profile, in dorsal view. The term aedeagus mesoconvex is here used to describe the aedeagus of most of species of Pseudisobrachium, in which it is dilated or even swollen medially, with apex and base usually somewhat contrastingly narrow (Fig. 7).

\section{Key}

I was not able to get enough information to include $P$. burchellanum and $P$. elegantulum in the key. I had no access to the material of $P$. graciliventre, $P$. hypogeum, P. mrazi, P. ogloblini, $P$. rapoporti, $P$. solenopsidicola and $P$. solenopsiphilum. All of these species are known only by females and they were keyed with information from the literature. The key is mostly based of the primary types. Thus, it represents only the initial taxonomic approach in order to prepare to a future revision of this genus since it is not include the variation of the species.

\section{TAXONOMY}

\section{Pseudisobrachium Kieffer, 1904}

Female generic characters. Body slender. Color usually castaneous. Palpal formula 5:3. Mandible with three or four apical teeth. Clypeus usually short, median lobe usually trapezoidal, sometimes angulate or rounded, median carina sometimes projecting far apical margin as tooth. Antenna short and thick, 13-segmented. Head longer than wide, few exceptions. Eye consisting of one facet, with few exception. Ocelli absent. Pronotal disc longer than wide, lateral margin usually straight in dorsal view; pronotal collar well developed. Wings and tegulae absent. Mesoscutum elongate, dorsally extending through entire length of mesopleuron. Notaulus and parapsidal furrow absent. Propodeal disc with subanterior constriction, anterior area usually strongly concave with lateral flank embracing posterior end of mesoscutum, posterior carina usually absent; propodeal spiracle laterad or dorsad. Mesopleuron bulging laterally. Mid tibia usually spinose. Metasoma usually not petiolate.

Male generic characters. Body slender. Color usually black or castaneous. Palpal formula 6:3. Mandible with three to five apical teeth. Clypeus well projected forward, median lobe usually trapezoidal, sometimes rectangular, angulate or rounded, median carina present, sometimes bifurcated apically. Antenna filiform, 13-segmented. Head usually longer than wide. Vertex convex or straight. Ocelli present. Pronotal disc ecarinate, usually wider than long, lateral margin strongly divergent posterad; pronotal collar well developed. Macropterous. Fore wing with costal, median and submedian cells closed; stigma well developed; radial vein long and tubular, discoidal and subdiscoidal veins not tubular, frequently weakly pigmented. Mesoscutum transverse. Notaulus variable, complete or incomplete, sometimes absent, well or ill developed. Scutellar groove usually wide. Propodeal disc usually with anterior triangular rugulose area (= basal triangle), median carina with variable extension, posterior carina usually absent or indistinguishable from back ground texture. Legs not swollen. Mid tibia not spinose. Metasoma usually not petiolate. Hypopygium with three short stalks. Genitalia. Paramere completely divided into ventral and dorsal arms; volsella with cuspis dome-shaped, digitus very wide with several teeth in dorsal surface, volsella with large grooved inner expansion (the vannus of Evans 1961); aedeagus elongate, usually mesoconvex, apodeme short; genital and basal ring V-shaped and completely turned downward.

Remarks. This is a large and difficult genus because the genitalia of all its species are much similar to each other, and one must rely on subtle and sometimes variable characters (Evans 1978). Frequently, it is a difficult tasks to translate into words such differences among the species. The most useful taxonomical characters are found on the head, especially number of mandibular teeth, shape and proportions of median clypeal lobe, size of eye and ocelli, vertex profile and head proportions. But the variations of conditions of these characters among the species are small, and to such a degree that it is often quite imprecise to simply try to express them with words. For instance, most of the Neotropical species have the median clypeal lobe trapezoidal, vertex slightly convex and temple profile diverging anterad in short gaps of variation. On the other hand, male genitalia, the most useful structures for taxonomic purposes in Bethylidae, are relative similar in different species because paramere, cuspis and aedeagus have few variations in shape and proportions.

\section{Key for males}

1. Median clypeal lobe quadrate or rectangular or nearly so, its lateral margins not converging apicad (Fig. 322) ............ 2

1 '. Median clypeal lobe angulate, rounded or trapezoidal, lateral margins converging apicad (Figs 16, 407)....

2(1). Median clypeal lobe quadrate, about as wide as long (Fig. 260) P. montivagum

2'. Median clypeal lobe rectangular, wider than long (Fig. 80)....3

3(2). Uppermost mandible tooth distinctly wider than two lower ones (Fig. 323) P. porteri

3'. Three mandibular teeth similar (Fig. 81) ......................... 4 4(3). Aedeagus constricted below apex (Fig. 82) ... P. clypeatum 4'. Aedeagus not constricted below apex (Fig. 137) P. deplanatum 
5(1). Median clypeal lobe subangulate or angulate (Fig. 12) 6

$5^{\prime}$. Median clypeal lobe rounded, trapezoidal or nearly so (Figs $278,364)$

... 8

6(5). Base of aedeagus wide, progressively widening apicad (Fig 26) P. apenesoides

6'. Base of aedeagus narrow, its lateral margins subparallel (Fig 18) 7

7(6). Mandible with five teeth of equal size (Fig. 17); head longer than wide, VOL more than 1.0x HE (Fig. 16); notaulus as strong and continuous line; propodeal disc longer than wide P. angulatum

7'. Mandibular lower tooth distinctly larger than others (Fig. 13); head wider than long, VOL less than 1.0x HE (Fig. 12); notaulus as series of not contiguous foveae; propodeal disc wider than long P. amplum

$8(5)$. Apical margin of median clypeal lobe concave (Figs 41 222) ... 9

8 '. Apical margin of median clypeal lobe straight or nearly so (Fig. 180) or convex (Fig. 29) 25

9(8). Apical margin median of clypeal lobe roundly concave (Fig. 41) .. 10

9'. Apical margin of median clypeal lobe angularly concave (Fig. 222) .. 18

10(9). Corner of lateral and apical margin of median clypeal lobe rounded (Fig. 41); VOL about 1.3x HE .......... P. blomi

$10^{\prime}$. Corner of lateral and apical margin of median clypeal lobe angled (Fig. 217, 326); VOL at most 1.1x HE .. 11

11(10). Ocellar triangle not so far from vertex crest, posterior ocellus distant from vertex crest at most $1.7 \mathrm{x}$ DAO, anterior ocellus not surpassing imaginary top eye line (Figs 217, 326)

12

11 '. Ocellar triangle far from vertex crest, posterior ocellus distant from vertex crest at least $2.7 \mathrm{x}$ DAO, anterior ocellus surpassing imaginary top eye line (Figs 116,187 ) ........ 16

12(11). Median clypeal lobe wide, median length shorter than apical margin width (Fig. 326); temples parallel ........... 13

$12^{\prime}$. Median clypeal lobe long, median length longer than apical margin width (Fig. 373); temples diverging anterad ..... 14

13(12). Head strongly converging posterad (Fig. 326); eye bulging ...... P. praecipum

13'. Head parallel behind eye (Fig. 217); eye not bulging ......

P. krombeini

14(13) Aedeagus entirely elliptical (Fig. 405) ...... P. ventriosum

14 '. Aedeagus mesoconvex (Fig. 46, 375) ..... 15

15(14). Aedeagus with apex wide and base elongate (Fig. 46)..

P. testaceipes

$15^{\prime}$. Aedeagus with apex not as wide as above and base short (Fig. 375) P. boliviense

16(11) Posterior ocellus reaching imaginary top eye line (Fig. 187); median carina of median clypeal lobe entirely bifurcating apicad; corner of apical margin of median clypeal lobe not forming tooth; hypopygium with posterior margin deeply concave (Fig. 189) P. haemorrhoidalis

16 '. Posterior ocellus not reaching imaginary top eye line (Fig. 111); base of median carina of median clypeal lobe not bifurcated; corner of apical margin of median clypeal lobe forming conspicuous tooth (Fig. 110); hypopygium with posterior margin convex (Fig. 112) 17

17(16). Mandible with four apical tooth (Fig. 111); base of median carina of median clypeal lobe narrow (Fig. 110); base of aedeagus elongate (Fig. 113) ..................... P. coxalis

17'. Mandible with five apical teeth (Fig. 117); base of median carina of median clypeal lobe thick (Fig. 116); base of aedeagus wide (Fig. 120) .

P. crassicornis

18(9). Aedeagus narrow, lateral margins straight or nearly so (Fig. 78) 19

18'. Aedeagus mesoconvex or bottle-shaped, lateral margins distinctly convex or nearly so (Fig. 152)

21

19(18). Aedeagus apex much higher than paramere apex (Fig. 78) P. chilense

19'. Aedeagus apex much lower than paramere apex (Fig. 225)

(19). Lateral margin of aedeagus weakly dilated submedially and weakly constricted subapically (Fig. 385); hypopygium with angular emargination (Fig. 382) ............. P. triacutum

20 '. Lateral margin of aedeagus straight (Fig. 225); hypopygium with rounded emargination (Fig. 224) ................. P. latum

21(18). Aedeagus bottle-shaped, ventral margin with pair of tridentate projection (Fig. 304); dorsal arm of paramere angled medially (Fig. 306) .P. pauxillum

21'. Aedeagus mesoconvex, ventral margin without pair of tridentate projection (Fig. 397); dorsal arm of paramere not angled medially (Fig. 398) 22

22(21). Aedeagus with pair of filaments turned upward (Fig. 152); dorsal arm of paramere constricted medially (Fig. 153)

P. filum

22 '. Aedeagus without such pair of filaments (Fig. 178); dorsal arm of paramere not constricted medially (Fig. 179) .... 22

23(22). Occipital carina high and sharp, conspicuously visible in dorsal view (Fig. 177); aedeagus slightly dilated basally (Fig. 178) P. formosum

23'. Occipital carina low, not visible in dorsal view (Fig. 32); aedeagus dilated medially (Fig. 35)

24

24(23). Ocelli large, distance among them less than their own diameter (Fig. 395); temple profile straight in dorsal view...

P. turbinatum

24'. Ocelli not large, distance among them more than their own diameter (Fig. 32); temple profile slight convex in dorsal view

P. beckeri

25(8). Median clypeal lobe rounded, lateral margins convex (Fig. 63) . 26

25'. Median clypeal lobe trapezoidal or nearly so, lateral margins straight (Fig. 202) . 30

Revista Brasileira de Zoologia 25 (4): 737-801, December, 2008 
26(25). Pronotal disc with strong and deep transverse groove paralleling posterior margin (Fig. 63); notaulus wide and foveolate P. calidum

26 '. Pronotal disc without such groove; notaulus of regular width and not foveolate

27(26). Mandible with large of equal size teeth (Fig. 279); anterior ocellus reaching imaginary top eye line (Fig. 278); hypopygium with inner surface with pair of teeth and posterior margin sharply emarginate (Figs 280-281); aedeagus with five pairs of apical lobes (Fig. 282)

P. optimum

27'. Mandible with lower tooth distinctly larger than upper ones (Fig. 320); anterior ocellus not reaching imaginary top eye line (Fig. 319); hypopygium without such modifications; aedeagus with one pair of apical lobes 28

28(27). Eye and ocelli small, VOL more than 1.2x HE, DAO less than 0.1x WF (Fig. 319) P. plaumanni

$28^{\prime}$. Eye and ocelli of regular size, VOL less than $0.7 \mathrm{x}$ HE, DAO more than $0.12 x$ WF (Fig. 344) ..... ... 29

29(28). Temple and vertex evenly convex (Fig. 344); aedeagus about $2.9 \mathrm{x}$ as long as wide, constricted subapically (Fig. 346) P. rotundum

$29^{\prime}$. Temple and vertex not evenly convex (Fig. 131); aedeagus about $2.2 \mathrm{x}$ as long as wide, without subapical constriction (Fig. 133) P. demissum

30(25). Mandible with three (Fig. 181) or four apical teeth (Fig. 21) .. 31

30'. Mandible with five apical teeth (Fig. 349) .................... 45

31(30). Mandible with three apical teeth ............................ 32

31'. Mandible with four apical teeth .................................... 38

32(31). Eye small, WF more than 1.5x HE and VOL more than HE (Fig. 180) . P. fuscicornis

32 '. Eye large or of regular size, WF less than $1.5 x$ HE and VOL smaller than HE (Figs 52, 285) 33

33(32). Upper tooth of mandible distinctly wider than median tooth (Fig. 53); apical margin of median clypeal lobe slightly convex (Fig. 52) ... 34

33 '. Upper tooth of mandible about as wide as width of median tooth (Fig. 203); apical margin of median clypeal lobe straight (Fig. 202) 35

34(33). Ocelli small, DAO less than 0.15x WF (Fig. 52) ..........

P. breviceps

34 '. Ocelli large, DAO at least $0.18 \mathrm{x}$ WF (Fig. 285)...P. pallidipes $35(33)$. Ocelli very large, DAO about $0.25 x$ WF; eye very large, WF less than HE (Fig. 202) P. illippum

$35^{\prime}$. Ocelli of regular size, DAO less than $0.25 \mathrm{x}$ WF; eye of regular size, WF more than HE (Fig. 364)

36(35). Teeth of mandible wide and in conspicuous oblique series (Fig. 365); median clypeal lobe wider than long (Fig. 364) P. superbum

36 '. Teeth of mandible narrow and not in oblique series (Fig. 331); median clypeal lobe longer than wide (Fig. 330).....37
37(36). Temples strongly diverging anterad (Fig. 330); posterior ocellus distant from vertex crest less than $0.5 \mathrm{x}$ DAO ........

P. pseudoobscurum

37'. Temples slightly diverging anterad (Fig. 155); posterior ocellus distant from vertex crest more than $1.5 x$ DAO ....

P. flavicornis

38(31). Ocelli large, DAO at least 0.25x WF (Fig. 20) .......... 39

$38^{\prime}$. Ocelli not so large as above, DAO less than $0.21 \mathrm{x}$ WF (Fig. 56) .. 41

39(38). Apical margin of median clypeal lobe convex (Fig. 20); thorax dorsum polished, contrasting with coriaceous frons; apex of aedeagus with small spines (Fig. 22) ....... P. apache

39'. Apical margin of median clypeal lobe straight (Fig. 89); thorax dorsum not contrasting with coriaceous frons; apex of aedeagus without spines (Fig. 91) ............................. 40

40(39). Eye bulging (Fig. 89); apex of aedeagus wide (Fig. 91)... P. comanche

40'. Eye not bulging (Fig. 158); apex of aedeagus narrow (Fig. 160) P. flavinervis

41(38). Eye small, VOL at least 1.0x HE (Fig. 257) .............. 42

$41^{\prime}$. Eye of regular size, VOL less than HE (Fig. 165)

P. flavinervis

42(41). Aedeagus with subapical dilation (Fig. 58); base of aedeagus long and nearly evenly wide ................. P. bruesi

42'. Aedeagus without subapical dilation (Fig. 7); base of aedeagus progressively widened ..................................... 43

43(42). VOL at least 1.3x HE (Fig. 257); aedeagus with base about as wide as apex (Fig. 259) .................. minutissimus

43'. VOL less than 1.3x HE (Fig. 254); aedeagus with base narrower than apex (Fig. 256) ........................................ 44

44(43). Ocellar triangle compact, distance among ocelli less than their diameters (Fig. 5) ................................ P. albipes

44 '. Ocellar triangle not compact, distance among ocelli more than their diameters (Fig. 254) ...................... P. minimum 45(30). Ocelli large, DAO at least 0.25x WF (Fig. 407) ........ 46 $45^{\prime}$. Ocelli of regular size, DAO less than $0.25 x$ WF (Fig. 348)...51 46(45). Aedeagus little dilated medially, lateral margin nearly straight (Fig. 409) P. werneri

46'. Aedeagus distinctly dilated, lateral margin convex (Fig. 31)

. 47

47(46). Aedeagus strongly dilated, lateral margin conspicuously convex (Fig. 31); apical margin of median clypeal lobe convex (Fig. 29) P. aztecum

47'. Aedeagus regularly dilated, lateral margin regularly convex (Fig. 402); apical margin of median clypeal lobe straight (Fig. 401)

48(47). Distance of posterior ocellus to eye top less than its own diameter (Fig. 399); anterior ocellus surpassing top eye line more than half of its diameter; posterior margin of prosternum with long spine ........................ P. uruguayense

$48^{\prime}$. Distance of posterior ocellus to eye top much more than its own diameter (Fig. 264); anterior ocellus not surpassing 
top eye line more than half of its diameter; posterior margin of prosternum without spine.

49

49(48). Anterior ocellus reaching imaginary top eye line (Fig. 264) P. nigriculum

$49^{\prime}$. Anterior ocellus not reaching imaginary top eye line (Fig. 268) 50

50(49). Eye bulging; frons narrow, WF much less than HE (Fig 298) P. parcum

50 '. Eye not bulging; frons wide, WH much more than HE (Fig. 268) P. obscurum

51(46). Ocellar triangle compact, distance between posterior ocelli clearly less than their own diameter .................... 52

51 '. Ocellar triangle not compact, distance between posterior ocelli more than their own diameter .. 55

52(51). Ocellar triangle removed from vertex crest, anterior ocellus reaching imaginary top eye line 53

52 '. Ocellar triangle not so removed from vertex crest, anterior ocellus not reaching imaginary top eye line .. 54

53(52). Ocelli nearly touching one to another (Fig. 348); posterior ocellus reaching imaginary top eye line; eye large, VOL about $0.6 \mathrm{x}$ HE P. rufopictum

53'. Ocelli not so close to one another (Fig. 72); posterior ocellus not reaching imaginary top eye line; VOL about $0.9 x$ HE... P. chacoense

54(52). Metasoma clearly petiolate; eye of regular size, VOL less than HE (Fig. 316) P. petiolatum

$54^{\prime}$. Metasoma not petiolate; eye small, VOL at least $1.0 \mathrm{x} \mathrm{HE}$ (Fig. 390) .. 55

55(54). Head evenly rounded above eye (Fig. 37) P. bisulcatum

$55^{\prime}$. Head not evenly rounded above eye (Fig. 390)

P. tucumanum

56(51). Anterior ocellus surpassing imaginary top eye line (Fig 70) P. castaneiceps

56 '. Anterior ocellus not surpassing imaginary top eye line (Fig 145) 57

57(56). Eye small, VOL at least 1.1x HE 58

57 '. Eye of regular size, VOL at most $1.05 x$ HE 64

58(57). Head orange, contrasting to mesosoma dark castaneous; mandible with apical teeth large and of equal size (Fig. 146). P. erythrocephalum

58 '. Head never orange; mandible with upper three teeth smaller than two lower ones (Fig. 194) 59

59(58). Head strongly converging posterad (Fig. 193); eye distinctly bulging ............................................... howdeni

59'. Head at most slightly converging posterad (Fig. 197); eye at most slightly bulging .. 60

60(59). Median clypeal lobe wide, apical margin longer than its median length (Fig. 197) .. 61

60 '. Median clypeal lobe narrow, apical margin shorter than its median length (Fig. 104) .. 62

61(60). Apical margin of median clypeal lobe straight (Fig. 197); upper three teeth of mandible very small, nearly fused (Fig.
198) P. hurdi

$61^{\prime}$. Apical margin of median clypeal lobe with conspicuous rounded tooth (Fig. 386); upper three teeth of mandible distinct (Fig. 387) P. tridens

62(60). Apical margin of median clypeal lobe with rounded tooth (Fig. 104); upper teeth of mandible wider than two teeth below it (Fig. 105) P. corvinum

62'. Apical margin of median clypeal lobe straight (Fig. 240); upper three teeth of mandible equally wide (Fig. 241)....63

63(62). Median clypeal lobe distinctly projected forward, median length $1.5 \mathrm{x}$ apical margin width (Fig. 240); VOL about $1.3 \mathrm{x}$ HE; paramere with ventral arm about $1.4 \mathrm{x}$ wider than dorsal arm (Fig. 243)

P. masoni

63'. Median clypeal lobe not distinctly projected forward, median length $1.5 \mathrm{x}$ apical margin width (Fig. 334); VOL about 1.15x HE; paramere with ventral arm about 2.0x wider than dorsal arm (Fig. 337) P. pulcherrimum

64(57). Apical margin of median clypeal lobe with well developed median tooth (Fig. 360) 65

64'. Apical margin of median clypeal lobe straight or slightly convex, but never forming conspicuous tooth (Figs 246, 367)

68

65(64). Apical margin of median clypeal lobe tridentate (Fig. 360) ........................................................ P. steinbachi

65'. Apical margin of median clypeal lobe as above (Fig. 205)..

66(65). Anterior ocellus distance from imaginary top eye line less than their own diameter (Fig. 205); pronotal disc with transverse subapical depression ..................... P. inchoatum

$66^{\prime}$. Anterior ocellus distance from imaginary top eye line more than their own diameter (Fig.48); pronotal disc without transverse depression 67

67(66). Aedeagus about 3.2x as long as wide (Fig.) 50; mandible with sublower tooth distinctly larger than upper three teeth (Fig. 49) P. brasiliense

67'. Aedeagus about $2.3 \mathrm{x}$ as long as wide (Fig. 102); mandible with upper four teeth of equal size (Fig. 101) 29. P. cooperi

68(64). Mandible with apical teeth large and of equal size (Fig. 247) P. micheneri

$68^{\prime}$. Mandible with upper three teeth distinctly smaller than two lower one (Fig. 294) . 69

69(68). Median carina of median clypeal lobe bifurcating from base to apical corner (Fig. 293) P. pallidum

$69^{\prime}$. Median carina of median clypeal lobe at most bifurcating just before apical margin (Fig. 312) ............................... 70

70(69). VOL less than 0.7x HE (Fig. 1) ................................. 71 70 '. VOL more than $0.7 x$ HE (Fig. 92) .................................. 73

71(70). DAO much more than 0.2x WF (Fig. 1); mandible with sublower teeth not distinctly larger than upper three ones (Fig. 2); aedeagus with subapical constriction (Fig. 3) ......

P. alayo

Revista Brasileira de Zoologia 25 (4): 737-801, December, 2008 
71'. DAO less than 0.2x WF (Fig. 312); mandible with sublower teeth distinctly larger than upper three ones (Fig. 313); aedeagus without subapical constriction (Fig. 314) ...... 72

72(71). Apical margin of median clypeal lobe straight (Fig. 312); frons polished P. perpunctatum

72 '. Apical margin of median clypeal lobe with small rounded tooth (Fig. 367); frons coriaceous P. ternarium

73(70). Temples in dorsal view parallel or nearly so, vertex corner not broadly rounded (Fig. 92) 74

73 '. Temples in dorsal view not parallel, vertex corner broadly rounded (Fig. 59) 75

74(73). Temples parallel (Fig. 92); aedeagus with base elongate, much longer than apex (Fig. 94); ventral margin of aedeagus with large lobe.... P. complanatum

$74^{\prime}$. Temples nearly parallel (Fig. 278); aedeagus with base about as long as apex (Fig. 282); ventral margin of aedeagus without lobe P. opimum

75(73). Fore wing without discoidal vein ............................ 76

75'. Fore wing with discoidal vein ...................................... 77

76(75). Mandible with sublower tooth distinctly larger than upper three teeth (Fig. 60); VOL about 0.8x HE (Fig. 59)...

P. brunneum

76'. Mandible with upper four teeth of equal size (Fig. 284); VOL about 1.0x HE (Fig. 283) ............................. P. otiosum

77(75). Aedeagus with subapical constriction in ventral view (Fig. 129) ... 78

77'. Aedeagus without subapical constriction in ventral (Fig. 378) 81

78(77). Frons polished; antenna thick, segment XI about $1.4 \mathrm{x}$ as long as wide; cuspis with subquadrate apical margin (Fig. 130) P. dalmati

$78^{\prime}$. Frons coriaceous; antenna not so thick, segment XI at least $2.0 \mathrm{x}$ as long as wide; cuspis rounded apical margin ..... 79

79(78). DAO more than 0.2x WF (Fig. 96); OOL less than WOT; paramere with ventral arm slightly wider than dorsal arm (Fig. 99)

P. concinum

79'. DAO at most 0.15x WF (Fig. 139); OOL much more than WOT; paramere with ventral arm more than $3.0 \mathrm{x}$ wider than dorsal arm (Fig. 142) ... 80

80(79). OOL about 2.2x WOT (Fig. 139); propodeal disc about $0.8 \mathrm{x}$ as wide as long P. distans

$80^{\prime}$. OOL about $1.5 \mathrm{x}$ WOT (Fig. 234); propodeal disc about as wide as long P. magnum

81(78). Aedeagus with apex conspicuously wider than base in ventral view (Fig. 378)

82

$81^{\prime}$. Aedeagus with apex at most as wide as base in ventral view (Fig. 215) 85

82(81). Aedeagus little dilated medially in ventral view, more than 5.0x as long as wide (Fig. 211) P. intentum

82 '. Aedeagus distinctly dilated, less than $4.0 \mathrm{x}$ as long as wide (Fig. 378)
83(81). Vertex corner somewhat subangulate, temple profile straight in dorsal view (Fig. 376); fore wing with discoidal vein not interstitial with median vein .........P. transversum

83'. Head evenly rounded behind eye (Fig. 340); fore wing with discoidal vein interstitial with median vein .................. 84

84(82). Antenna thick, segment XI 2.0x as long as wide; frons polished; notaulus short .............................. P. albitinctum

$84^{\prime}$. Antenna with segment XI about $1.5 \mathrm{x}$ as long as wide; frons strongly coriaceous; notaulus complete .......P. rettenmeyeri

85(84). Aedeagus much dilated medially in ventral view, less than $2.5 \mathrm{x}$ as long as wide (Fig. 215) $P$. jamaicense

$85^{\prime}$. Aedeagus regularly dilated medially in ventral view, 3.0$3.7 \mathrm{x}$ as long as wide (Fig. 86) ......................................... 86

86(85). Frons polished; notaulus nearly complete; specimen small, around $2.2 \mathrm{~mm}$ long ............................... P. collinum

86 '. Frons coriaceous; notaulus short; specimen large, more than $3.0 \mathrm{~mm}$ long 87

87(86). Posterior ocellus distant from vertex crest much more than DAO (Fig. 122) 88

$87^{\prime}$. Posterior ocellus distant from vertex crest at most 1.0x DAO (Fig. 66) 89

88(87). Discoidal vein of fore wing interstitial with median vein; mesopleuron with polished callus P. cuspidatum

$88^{\prime}$. Discoidal vein of fore wing not interstitial with median vein; mesopleuron with coriaceous callus

P. michoacanum

89(87). Inner margin of eye convergent anterad in dorsal view (Fig. 66); antennal segment XI about $1.2 \mathrm{x}$ as long as wide; fore wing with discoidal vein nearly interstitial with median vein P. capixabum

89'. Inner margin of eye parallel in dorsal view (Fig. 356); antennal segment $\mathrm{XI}$ about $1.7 \mathrm{x}$ as long as wide; fore wing with discoidal vein not interstitial with median vein ...... P. stangei

\section{Key for females}

1 Mandible with three apical teeth (Fig. 109) ........................ 2

1 '. Mandible with four apical teeth (Fig. 88) ........................ 7

2(1). Median clypeal lobe trapezoidal (Fig. 232) .................... 3

2'. Median clypeal lobe not trapezoidal (Fig. 186) ................ 6

3(2). Apical margin of median clypeal lobe concave (Fig. 108)...4

3'. Apical margin of median clypeal lobe nearly straight (Fig. 232) or convex (Fig. 338)

4(3). Apical margin of median clypeal lobe with median tooth (Fig. 108); mandible wider apically (Fig. 109)

P. costaricarum

4'. Apical margin of median clypeal lobe without median tooth (Fig. 220); mandible not wider apically (Fig. 221)

P. laticeps

5(3) Apical margin of median clypeal lobe convex (Fig. 338); pronotal disc much longer than mesoscutum (Fig. 339) .. P. rapoporti 
5'. Apical margin of median clypeal lobe nearly straight, slightly produced where median carina reaching apical margin (Fig. 232); pronotal disc as long as mesoscutum (Fig. 232) ......

P. luisae

6(2) Median clypeal lobe rounded (Fig. 199); pronotal disc much longer $(1.38 \mathrm{x})$ than mesoscutum (Fig. 201) .... P. hypogeum

6'. Median clypeal lobe angulate (Fig. 186); pronotal disc not as long as above, 1.12x longer than mesoscutum (Fig. 186)

P. graciliventre

7(1) Median clypeal lobe angulate (tentatively) (Fig. 263) ... 8

7'. Median clypeal lobe trapezoidal (Fig. 411) .................... 10

8(7) Sides of head diverging anteriorly (Fig. 263) ........P. mrazi

8'. Sides of head mostly parallel (Fig. 352) ............................ 9

9(8) Head quadrate (Fig. 263); lateral margin of pronotal disc convex (Fig. 263); propodeal disc with posterior carina ...

P. solenopsicola

9'. Head longer than wide (Fig. 267); lateral margin of pronotal disc straight (Fig. 267); propodeal disc without posterior carina P. ogloblini

10(7) Apical margin of median clypeal lobe concave (Fig. 371)

.. 11

$10^{\prime}$. Apical margin of median clypeal lobe straight (Fig. 143) or slightly convex (Fig. 355)

11(10) Specimens large, body length about $6.0 \mathrm{~mm}$; metasoma not petiolate P. terresi

11'. Specimens small, body length about $3.0 \mathrm{~mm}$; metasoma with short petiolate. .. 12

12(11) Malar space as long as eye length (Fig. 411); occipital carina visible in dorsal view (Fig. 411) .................. P. zeteki

12'. Malar space long than eye length; occipital carina not visible in dorsal view (Fig. 192) P. haywardi

13(10) Apical margin of median clypeal lobe slightly convex (Fig. 355) P. solenopsiphilum

13'. Apical margin of median clypeal lobe straight (Fig. 143)

14(13) Apical margin of median clypeal lobe with median tooth (Fig. 87) .... .. 15

$14^{\prime}$. Apical margin of median clypeal lobe without median tooth (Fig. 143) ..... .. 16

15(14) median clypeal tooth large (Fig. 87); malar space about 2.0x as long as eye length (Fig. 87); pronotal disc and mesoscutum not separated by deep and wide suture; metasoma not petiolate P. colombianum

15'. Median clypeal tooth small (Fig. 184); malar space less than $2.0 \mathrm{x}$ as long as eye length (Fig. 184); pronotal disc and mesoscutum separated by deep and wide suture; metasoma with short petiolate. P. gigas

16(13) Anterior half of head with punctures in longitudinal channeled lines $P$. distinguendum

$16^{\prime}$. Anterior half of head without such lines 17

17(16) Frons without unpunctuated median longitudinal band P. manni
17'. Frons with unpunctuated median longitudinal band.. 18

18(17) Body length about $2.0 \mathrm{~mm}$; malar space longer than eye length (Fig. 272); Metasoma with short petiole

P. obscurum

18'. Body length about $4.0 \mathrm{~mm}$; malar space about as long as eye length metasoma not petiolate P. merklei

\section{Pseudisobrachium alayoi Evans, 1969 \\ Figs $1-4$}

Holotype, male. CUBA, Cienfuegos, Las Villas, Soledad, V.1968, a la luz, (ACC, \#7.001070).

Descriptions. Male. Length $4.3 \mathrm{~mm}$. Black, fore wing subhyaline. Mandible with five apical teeth, upper four nearly of equal size. Clypeus with subtrapezoidal median lobe, median carina bifurcated apically. Antennal segment XI 1.7x as long as wide, pubescence of basal flagellomeres subappressed and short, with erect setae outstanding pubescence. Frons polished, punctures shallow, small and numerous. WH 0.99x LH, WF 0.53x WH, WF 1.02x HE, DAO 0.24x WF, OOL 0.79x WOT, posterior ocellus distant from vertex crest 1.0x DAO, anterior margin of anterior ocellus closed to eye top imaginary line. Vertex convex, corner rounded. VOL $0.64 \mathrm{x}$ HE. Notaulus absent on posterior third of mesoscutum. Parapsidal furrow complete. Propodeal disc $0.91 \mathrm{x}$ as wide as long, $0.61 \mathrm{x}$ as high as long, median carina incomplete, posterior carina absent, basal triangle inconspicuous; declivity strigulate. Mesopleuron with callus. Fore wing with discoidal vein unpigmented, interstitial with median vein. Genitalia: paramere with ventral arm $3.5 \mathrm{x}$ wider than dorsal; aedeagus mesoconvex, its apex not reaching apex of dorsal arm of paramere.

\section{Pseudisobrachium albipes (Ashmead, 1894) Figs 5-7}

Holotype, male. SAINT VINCENT: W. I., H. H. Smith col. (USNM, \#2493)

Descriptions. Male. Length $3.37 \mathrm{~mm}$. Dark castaneous. Mandible with four nearly of equal size apical teeth. Clypeus with subtrapezoidal median lobe. Antenna with segment XI 1.67x as long as wide, pubescence of basal flagellomeres subappressed, with some long suberect setae outstanding pubescence. Eye longhaired. Frons coriaceous, punctures shallow. WH 0.93x LH; WF 0.68x WH, WF 1.48x HE, DAO 0.16x WF, OOL 1.63x WOT, posterior ocellus distant from vertex crest $1.12 x$ DAO. Vertex convex, corner broadly rounded. VOL 1.0x HE. Notaulus inconspicuous, present on anterior third of mesoscutum. Propodeal disc $0.87 \mathrm{x}$ as wide as long, $0.67 \mathrm{x}$ as high as long, median carina incomplete, basal triangle ill defined, posterior carina indistinguishable; declivity weakly coriaceous; lateral of propodeum weakly coriaceous. Mesopleuron with polished large callus. Fore wing with discoidal vein weakly pigmented, interstitial with median vein. Genitalia: paramere with ventral arm 3.0x wider than dorsal; aedeagus mesoconvex, its apex reaching beyond apex of dorsal arm of paramere, base narrow and long. 

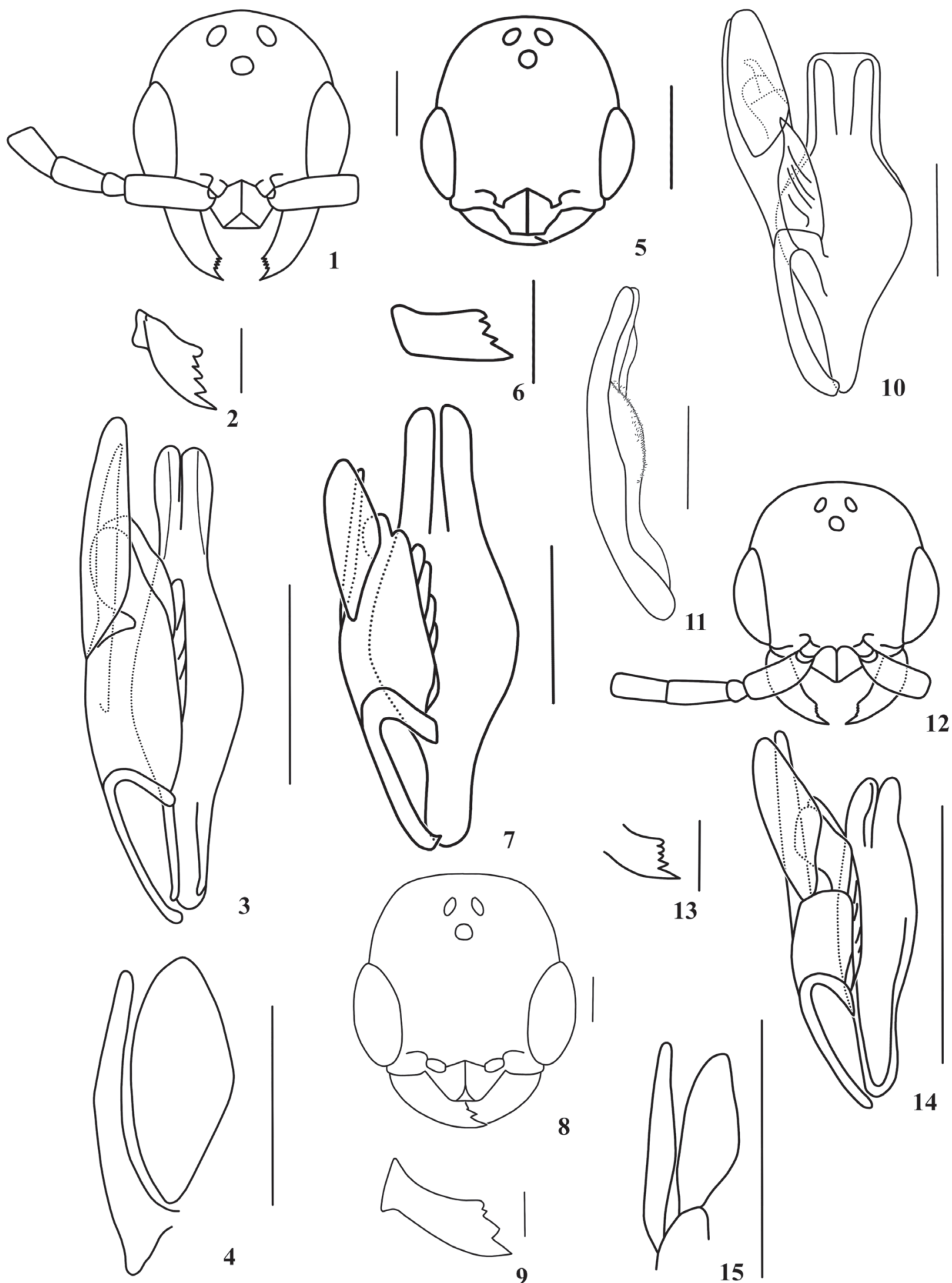

13
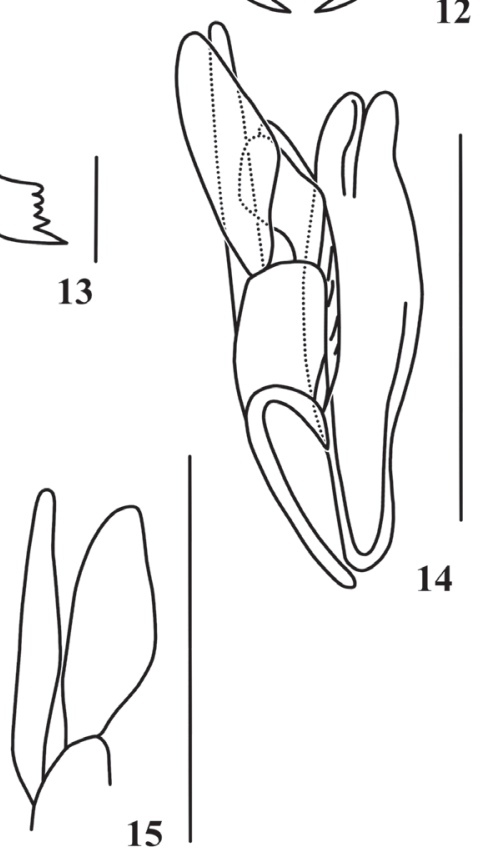

14

Figures1-15. Pseudisobrachium alayoi holotype male: (1) head, dorsal; (2) mandible, frontal; (3) genitalia, ventral; (4) paramere, full; $P$. albipes holotype male: (5) head, dorsal; (6) mandible, frontal; (7) genitalia, ventral; P. albitinctum holotype male: (8) head, dorsal; (9) mandible, frontal; (10) genitalia, ventral; (11) aedeagus, lateral; P. amplum holotype male: (12) head, dorsal; (13) mandible, frontal; (14) genitalia, ventral; (15) paramere, full. Bar $=250 \mu \mathrm{m}$. 


\section{Pseudisobrachium albitinctum Evans, 1966 Figs 8-11}

Holotype, male. C[OSTA] R[ICA], Alajuela, La Fortuna, 600', 18.II.[19]64, H. E. Evans col. (MCZH, \#31242).

Descriptions. Male. Length $6.07 \mathrm{~mm}$. Black. Mandible with five apical teeth, median and subdorsal teeth small, upper three smaller than sublower. Clypeus with subtrapezoidal median lobe, apical margin somewhat straight. Antenna thick, segment XI 2.0 as long as wide, pubescence of basal flagellomeres subappressed, with some long erect setae outstanding pubescence. Eye long-haired. Frons polished, punctures conspicuous. WH 1.03x LH, WF 0.64x WH, WF 1.4x HE, DAO 0.15x WF, ocellar triangle compact, OOL 1.52x WOT, posterior ocellus distant from vertex crest $1.33 x$ DAO. Vertex slightly convex, corner broadly rounded. VOL $0.89 x$ HE. Notaulus conspicuous, short, present on anterior third of mesoscutum. Propodeal disc 0.96x as wide as long, $0.63 \mathrm{x}$ as high as long, median carina incomplete, strongly rugulose, posterior carina indistinguishable; declivity strigulate; lateral of propodeum polished. Mesopleuron with polished large callus. Fore wing with discoidal vein weakly pigmented, interstitial with median vein. Genitalia: paramere with ventral arm 2.0x wider than dorsal; aedeagus mesoconvex, apex wide, not reaching apex of dorsal arm of paramere.

\section{Pseudisobrachium amplum Waichert \& Azevedo, 2004 Figs 12-15}

Holotype, male. BRAZIL, E[spírito] S[anto], S[anta] Teresa, Est[ação] Biol[ógica de] Santa Lúcia, 23.IV.2001, sweeping, C.O. Azevedo \& R. Kawada col. (UFES).

Descriptions. Male. Length $3.6 \mathrm{~mm}$. Black; fore wing subhyaline. Mandible with five apical teeth, upper four small and nearly of equal size. Clypeus with subangulate median lobe, median carina high and arched in profile. Antennal segment XI $2.9 \mathrm{x}$ as long as wide; pubescence of basal flagellomeres suberect, with some erect setae outstanding pubescence. Frons mostly polished, punctures conspicuous, area between torullus and eye entirely unpunctuated. WH 1.03x LH, WF 0.61x WH, WF 1.24x HE, DAO 0.14x WF, OOL 1.64x WOT, posterior ocellus distant from vertex crest $1.75 x$ DAO. Vertex convex. VOL $0.67 x$ HE. Temple slightly divergent anterad. Notaulus as series of not contiguous foveae. Propodeal disc $1.16 \mathrm{x}$ as wide as long, $0.65 \mathrm{x}$ as high as long, basal triangle large, median carina incomplete, lateral of propodeum polished centrally. Mesopleuron with large polished callus. Fore wing with discoidal vein weakly pigmented basally, interstitial with median vein. Genitalia: paramere with ventral arm $2.1 \mathrm{x}$ wider than dorsal arm; aedeagus mesoconvex and short, apex wide, not reaching apex of dorsal arm of paramere.

\section{Pseudisobrachium angulatum Evans, 1964} Figs 16-19

Holotype, male. [PERU], Iquitos, III-IV.1931, R. C. Shannon col. (USNM, \#64994). Genitalia slide \#HE497.
Descriptions. Male. Length $3.6 \mathrm{~mm}$. Black. Mandible broad apically, with five large of equal size apical teeth. Clypeus with angulate median lobe. Antennal segment XI 2.3 as long as wide, pubescence subappressed, longer than half of diameter of basal flagellomeres, with some long erect setae outstanding pubescence. Eye long-haired. Frons polished, punctures conspicuous. WH 0.93x LH, WF 0.6x WH, WF 1.18x HE, DAO 0.15x WF, ocellar triangle very compact, OOL $1.54 \mathrm{x}$ WOT, posterior ocellus distant from vertex crest $1.67 \mathrm{x}$ DAO. Vertex convex, corner broadly rounded. VOL 0.82x HE. Notaulus conspicuous, convergent and incomplete posteriorly. Propodeal disc $0.82 \mathrm{x}$ as wide as long, $0.57 \mathrm{x}$ as high as long, median carina incomplete, strongly strigulate, posterior carina indistinguishable, with pair of sublateral carinae incomplete anteriorly; declivity strigulate. Mesopleuron with polished callus. Fore wing with discoidal vein weakly pigmented basally, interstitial with median vein. Genitalia: paramere with ventral arm 2.0x wider than dorsal; aedeagus mesoconvex, its apex not reaching apex of dorsal arm of paramere.

\section{Pseudisobrachium apache Evans, 1961 Figs $20-23$}

Holotype, male. [U.S.A.], Ariz[ona], Santa Cruz Co., Pena Blanca, 3950', 24.VIII.1959, at light, H. E. Evans col. (MCZ, \#30288).

Descriptions. Male. Length $4.86 \mathrm{~mm}$. Dark castaneous, head nearly black. Mandible with four apical teeth, uppermost broad, upper two smaller than sublower. Clypeus with subtrapezoidal median lobe, apical margin slightly convex, median carina bifurcated apically. Antenna thick, segment XI 1.22 as long as wide, pubescence of basal flagellomeres subappressed, with some long erect setae outstanding pubescence. Eye longhaired. Frons coriaceous, punctures shallow. WH 1.01x LH, WF 0.61x WH, WF 1.08x HE, ocelli large, DAO 0.26x WF, OOL 0.59x WOT, posterior ocellus distant from vertex crest $0.5 \mathrm{x}$ DAO, anterior margin of anterior ocellus reaching eye top imaginary line. Vertex convex, corner rounded. VOL 0.51x HE. Notaulus narrow, incomplete posteriorly. Propodeal disc $0.73 \mathrm{x}$ as wide as long, $0.54 \mathrm{x}$ as high as long, median carina incomplete, weakly strigulate at basal triangle, otherwise coriaceous, posterior carina absent. Mesopleuron with weakly coriaceous callus. Fore wing without discoidal vein. Genitalia: paramere with ventral arm 2.16x wider than dorsal; aedeagus mesoconvex, apex with few small spines, not reaching apex of dorsal arm of paramere.

Remarks. The apical margin of median clypeal lobe of one paratype is straight.

\section{Pseudisobrachium apenesoides Waichert \& Azevedo, 2004} Figs 24-28

Holotype, male. BRAZIL, E[spírito] S[anto], Cariacica, Res[erva] Biol[ógica de] Duas Bocas, 22.X.1996, sweeping, H. S. Sá col. (UFES).

Descriptions. Male. Length $6.9 \mathrm{~mm}$. Black; fore wing subhyaline. Mandible with five apical teeth, upper four small 

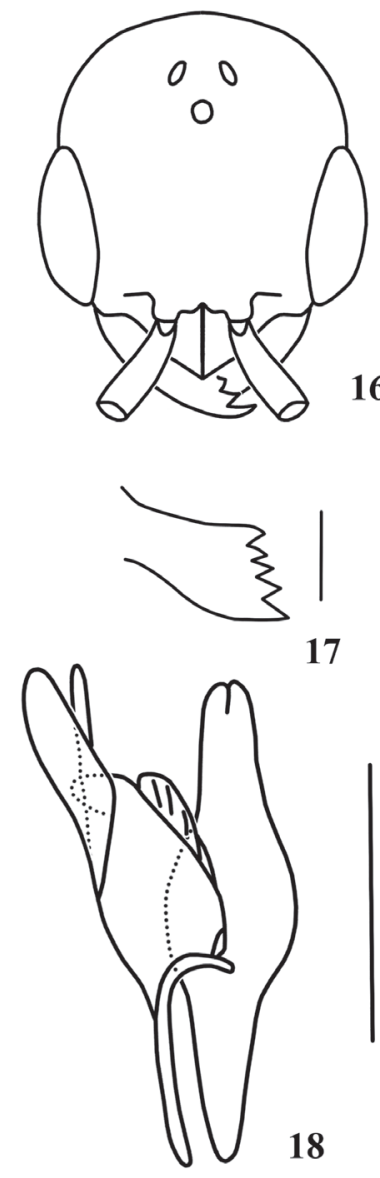

16
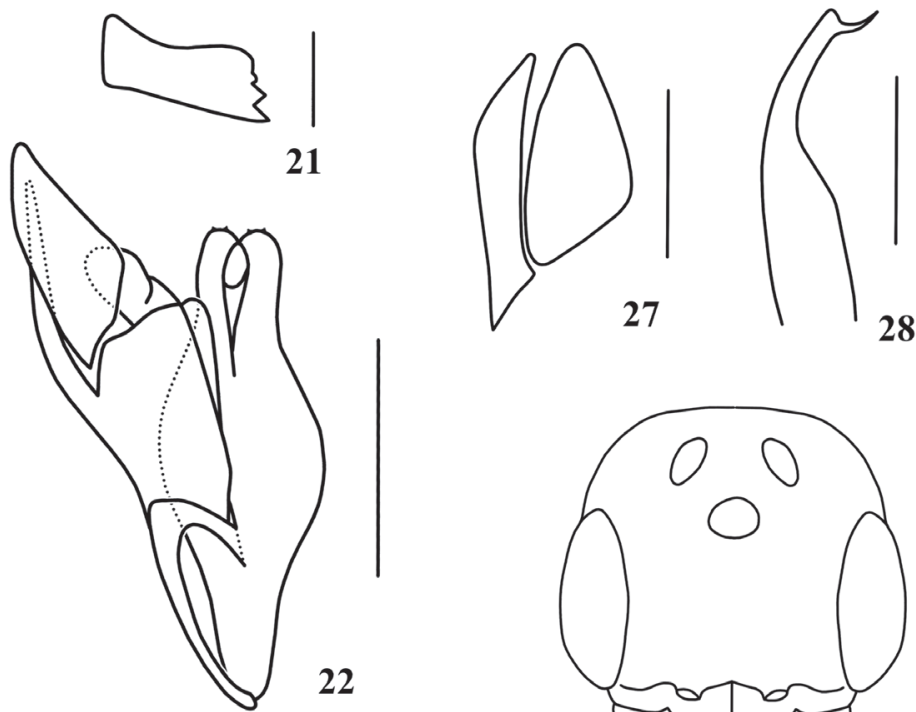

17
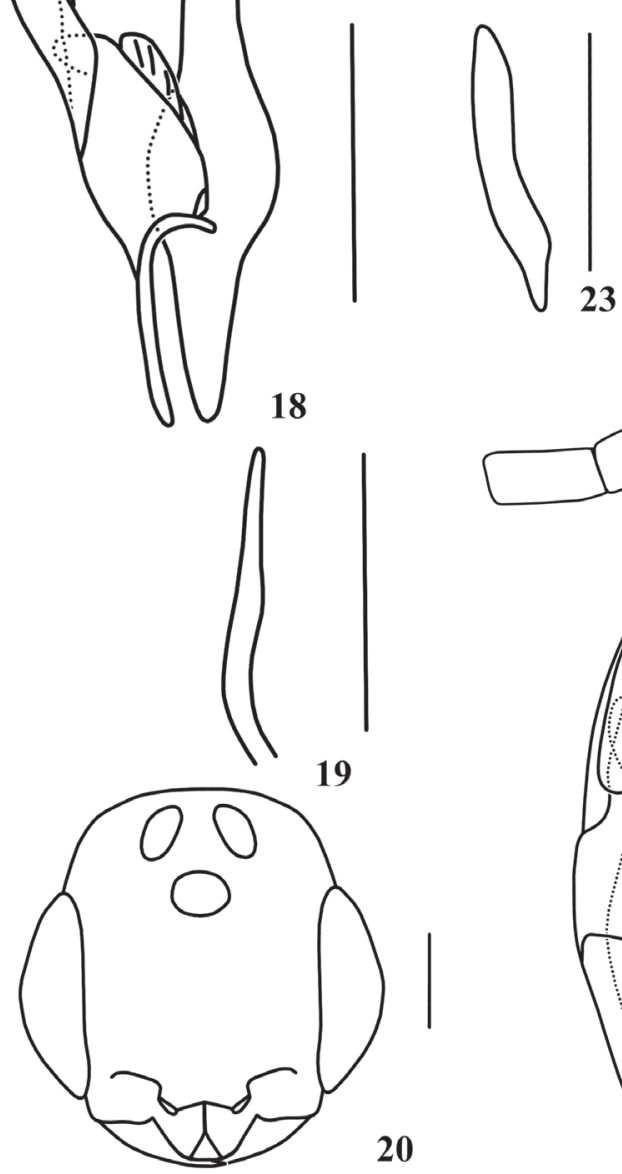

22

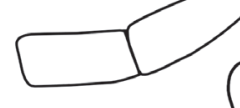

24

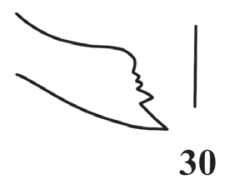

29

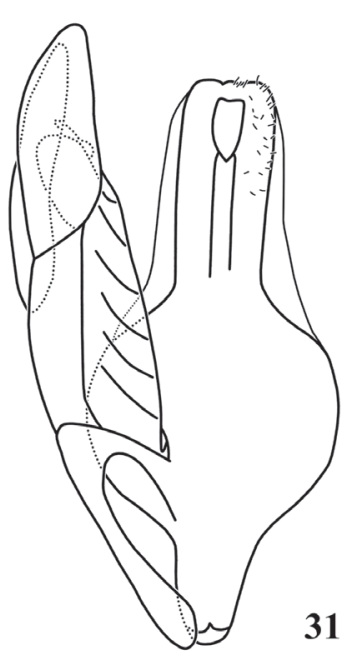

Figures 16-31. Pseudisobrachium angulatum holotype male: (16) head, dorsal; (17) mandible, frontal; (18) genitalia, ventral; (19) dorsal arm of paramere, full; P. apache holotype male: (20) head, dorsal; (21) mandible, frontal; (22) genitalia, ventral; (23) dorsal arm of paramere, full; P. apenesoides holotype male: (24) head, dorsal; (25) mandible, frontal; (26) genitalia, ventral; (27) paramere, full; (28) aedeagus, lateral; P. aztecum holotype male: (29) head, dorsal; (30) mandible, frontal; (31) genitalia, ventral. Bar $=250 \mu \mathrm{m}$. 
and nearly of equal size. Clypeus with subangulate median lobe, median carina complete, high and angled in profile. Antennal segment XI $3.3 \mathrm{x}$ as long as wide, pubescence of basal flagellomeres short, appressed, with some erect setae outstanding pubescence. Frons polished, punctures conspicuous. WH 1.0x LH, WF 0.57x WH, WF 1.15x HE; DAO 0.13x WF; OOL $1.13 \mathrm{x}$ WOT, posterior ocellus distant from vertex crest $2.0 \mathrm{x}$ $\mathrm{DAO}$, anterior margin of anterior ocellus nearly reaching eye top imaginary line. Vertex broadly convex. VOL 0.52x HE. Temple divergent anterad. Notaulus strongly impressed, almost complete. Propodeal disc $1.03 \mathrm{x}$ as wide as long, $0.62 \mathrm{x}$ as high as long, entirely rugulose, median carina complete, posterior carina unusually concave, declivity and lateral of propodeum mostly polished. Mesopleuron with polished callus. Fore wing with discoidal vein weakly pigmented basally, interstitial with median vein. Genitalia: paramere with ventral arm $2.3 \mathrm{x}$ wider than dorsal arm, dorsal arm angled in lateral view; aedeagus oval, wide, its apex not reaching apex of dorsal arm of paramere, with pair of filaments.

\section{Pseudisobrachium aztecum Evans, 1961} Figs 29-31

Holotype, male. MEX[ICO], Mor[elos], Cuernavaca, 5500', 6.VI.[19]59, H. E. Evans col. (MCZH, \#30279).

Descriptions. Male. Length $5.9 \mathrm{~mm}$. Dark castaneous, head nearly black. Mandible with five apical teeth, uppermost broad, upper three teeth smaller than sublower. Clypeus with subtrapezoidal median lobe, apical margin slightly convex, median carina bifurcated apically. Antenna thick, segment XI 1.5 as long as wide, pubescence of basal flagellomeres appressed, with some long erect setae outstanding pubescence. Eye longhaired. Frons weakly coriaceous, punctures conspicuous. WH 1.14x LH, WF 0.63x WH, WF 1.24x HE, ocelli large, DAO 0.25x WF, OOL $0.56 \mathrm{x}$ WOT, posterior ocellus distant from vertex crest $1.17 \mathrm{x}$ DAO, anterior margin of anterior ocellus reaching eye top imaginary line. Vertex slightly convex, corner rounded. VOL 0.6x HE. Notaulus conspicuous, absent on posterior third of mesoscutum. Propodeal disc $0.86 \mathrm{x}$ as wide as long, $0.54 \mathrm{x}$ as high as long, median carina incomplete, basal triangle well defined, posterior carina indistinguishable. Mesopleuron with polished callus. Fore wing with discoidal vein unpigmented, not interstitial with median vein. Genitalia: paramere with ventral arm 2.0x wider than dorsal; aedeagus mesoconvex, much dilated, apex wide and long, not reaching apex of dorsal arm of paramere, base short.

\section{Pseudisobrachium beckeri Evans, 1969 Figs 32-36}

Holotype, male. JAMAICA, 4000 feet, Hardwar Gap, 25.VII.1966, Howden \& Becker col. (CNCI, \#10992).

Descriptions. Male. Length $2.8 \mathrm{~mm}$. Dark castaneous, head black. Mandible with five apical teeth, upper three teeth smaller than sublower. Clypeus with trapezoidal median lobe, apical margin angularly concave. Antennal segment XI 1.4 as long as wide, pubescence suberect, longer than half of diameter of basal flagellomeres, with some long erect setae outstanding pubescence. Eye small. Frons coriaceous, punctures small. WH 0.94x LH, WF 0.68x WH, WF 1.5x HE, DAO 0.15x WF, OOL $1.5 \mathrm{x}$ WOT, posterior ocellus distant from vertex crest $1.14 \mathrm{x}$ DAO. Vertex rounded. VOL 1.1x HE. Notaulus present on anterior third of mesoscutum, well impressed. Propodeal disc $0.9 \mathrm{x}$ as wide as long, $0.69 \mathrm{x}$ as high as long, median carina incomplete, posterior carina inconspicuous. Mesopleuron with coriaceous callus. Fore wing with discoidal vein very weakly pigmented basally, interstitial with median vein. Genitalia: paramere with ventral arm $2.67 \mathrm{x}$ wider than dorsal arm; aedeagus mesoconvex, apex rounded without emargination, aligned with apex of dorsal arm of paramere.

\section{Pseudisobrachium bisulcatum Evans, 1969 Figs $37-40$}

Holotype, male. JAMAICA, 4000 feet, Hardwar Gap, 10.VII.1966, Howden \& Becker col. (CNCI, \#10987).

Descriptions. Male. Length $5.0 \mathrm{~mm}$. Black. Mandible with five apical teeth, upper three teeth smaller than sublower. Clypeus with trapezoidal median lobe, apical margin slightly concave. Antennal segment XI 2.25 as long as wide, pubescence of basal flagellomeres subappressed, with some long erect setae outstanding pubescence. Eye small. Frons strongly coriaceous, punctures small. WH 0.98x LH, WF 0.68x WH, WF 1.7x HE, DAO 0.16x WF, OOL 1.5x WOT, posterior ocellus distant from vertex crest $1.06 x$ DAO. Vertex convex, corner broadly rounded. VOL 1.1x HE. Notaulus strong and complete. Propodeal disc as wide as long, $0.64 \mathrm{x}$ as high as long, median carina almost complete, posterior carina absent. Mesopleuron with coriaceous callus. Fore wing with discoidal vein weakly pigmented basally, not interstitial with median vein. Genitalia: paramere with ventral arm $2.5 x$ wider than dorsal arm; aedeagus mesoconvex, its apex not reaching apex of dorsal arm of paramere, base gradually widened apicad.

\section{Pseudisobrachium blomi Evans, 1961 Figs $41-43$}

Holotype, male. MEX[ICO], Chiap[as], San Cristobal [de] I[as] Casas, 7500', 6.VI.[19]59, H. E. Evans col. (MCZH, \#30265).

Descriptions. Male. Length $6.66 \mathrm{~mm}$. Black. Mandible with five apical teeth, upper three teeth smaller than sublower. Clypeus with subtrapezoidal median lobe, apical margin concave, corner broadly rounded, median carina bifurcated apically. Antenna thick, segment XI 2.0 as long as wide, pubescence of basal flagellomeres subappressed, with some long erect setae outstanding pubescence. Eye small, long-haired. Frons polished, punctures small. WH 0.9x LH, WF 0.66x WH, WF 1.5x HE, DAO 0.09x WF, OOL 1.72x WOT, space between posterior ocel- 

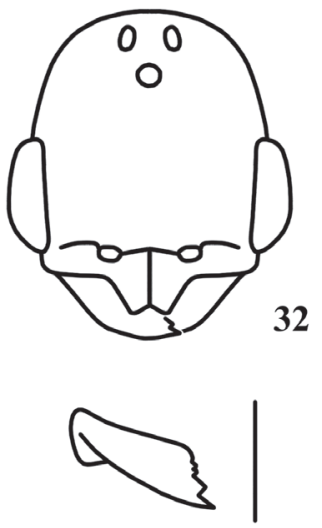

33

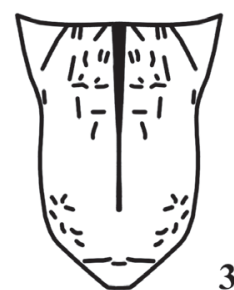

34

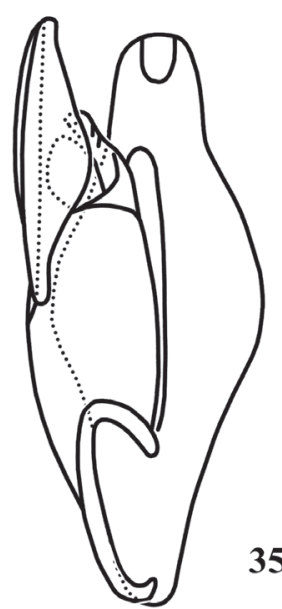

35

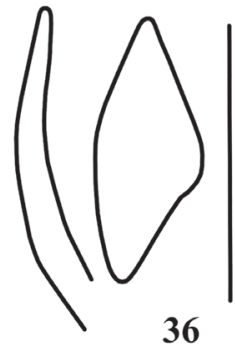

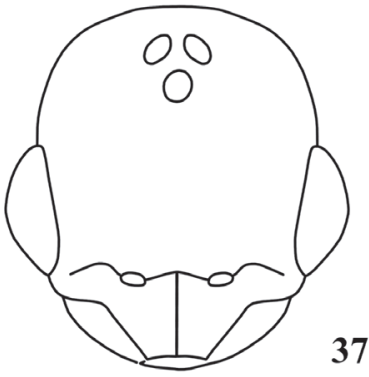

37

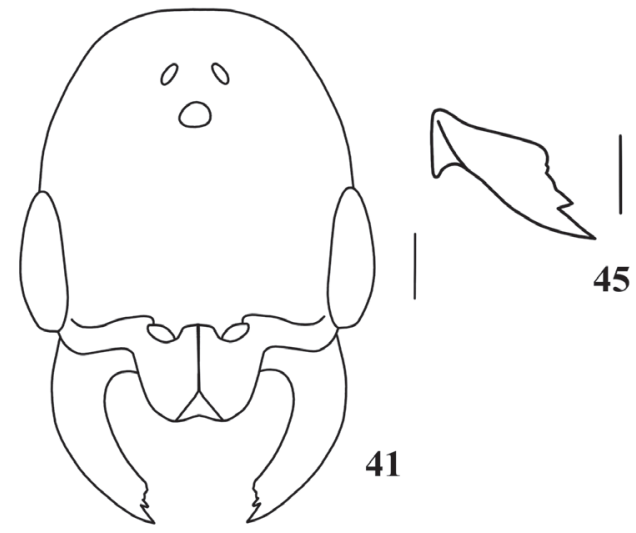

38
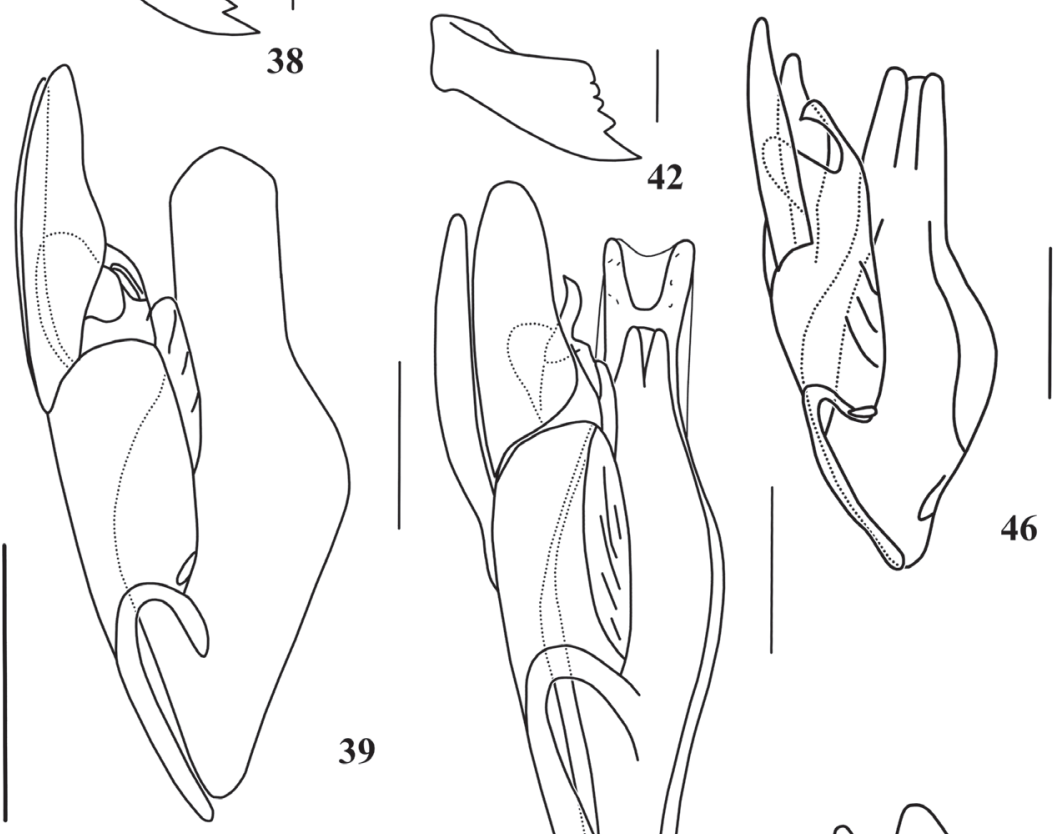

43

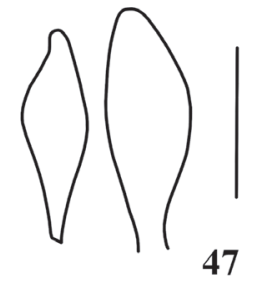

47

Figures 32-47. Pseudisobrachium beckeri holotype male: (32) head, dorsal; (33) mandible, frontal; (34) propodeum, dorsal. 35) genitalia, ventral; (36) paramere, full; P. bisulcatum holotype male: (37) head, dorsal; (38) mandible, frontal; (39) genitalia, ventral; (40) paramere, full; P. blomi holotype male: (41) head, dorsal; (42) mandible, frontal; (43) genitalia, ventral; P. boliviense holotype male: (44) head, dorsal; (45) mandible, frontal; (46) genitalia, ventral; (47) paramere, full. Bar $=250 \mu \mathrm{m}$. 
lus and vertex crest $1.77 \mathrm{x}$ DAO, with finely rugulose. Vertex slightly convex, corner broadly rounded. VOL $1.3 x$ HE. Notaulus conspicuous, except posteriorly. Propodeal disc $0.92 \mathrm{x}$ as wide as long, $0.67 \mathrm{x}$ as high as long, median carina incomplete, strongly rugulose, posterior carina indistinguishable; declivity strigulate. Mesopleuron with polished large callus. Fore wing with discoidal vein weakly pigmented basally, not interstitial with median vein. Genitalia: paramere with ventral arm $2.0 \mathrm{x}$ wider than dorsal, dorsal arm shorter; aedeagus mesoconvex, apex with deep median emargination, not reaching apex of dorsal arm of paramere.

Remarks. The fine striae behind the ocelli in this species is an unusual feature.

\section{Pseudisobrachium boliviense Kieffer, 1910 Figs 44-47}

Holotype, male. BOLIVIA, Mapiri, Staudinger V. col. (ZMHB, \#186).

Descriptions. Male. Length $5.8 \mathrm{~mm}$. Black, fore wing subhyaline. Mandible with five apical teeth, upper three teeth smaller than sublower. Clypeus with bidentate median lobe, apical margin concave, corner angled, median carina inconspicuous and bifurcated apically. Antennal segment XI 1.6x as long as wide, pubescence of basal flagellomeres appressed with erect setae outstanding pubescence. Frons polished, punctures conspicuous. WH 0.86x LH, WF 0.65x WH, WF 1.23x HE, DAO $0.14 \mathrm{x}$ WF, OOL $1.5 \mathrm{x}$ WOT, posterior ocellus distant from vertex crest $1.67 \mathrm{x}$ DAO. Vertex convex, corner rounded. VOL $0.75 \mathrm{x}$ HE. Notaulus well-impressed. Parapsidal furrow well-impressed, absent anteriorly. Propodeal disc $1.0 \mathrm{x}$ as wide as long, $0.8 \mathrm{x}$ as high as long, median carina incomplete, basal triangle not defined, posterior carina indistinguisable from strigulation; declivity strigulate. Mesopleura with large callus. Fore wing with discoidal vein nearly unpigmented, not interstitial with median vein. Genitalia: paramere with ventral arm $1.5 \mathrm{x}$ wider than dorsal, dorsal arm much wider medially; aedeagus mesoconvex, its apex not reaching apex of dorsal arm of paramere, base short, apical half arched downward.

\section{Pseudisobrachium brasiliense Kieffer, 1910 Figs 48-51}

Holotype, male. BRAZIL, Pará, Baker col. (CASC, \#9692).

Descriptions. Male. Length $4.1 \mathrm{~mm}$. Black. Mandible with five apical teeth, upper three smaller than sublower. Clypeus with trapezoidal median lobe, apical margin convex, median carina bifurcated apically. Antennal segment XI 1.8 as long as wide, pubescence of basal flagellomeres conspicuous and appressed, with some long erect setae outstanding pubescence. Frons coriaceous, punctures small. WH 0.92x LH, WF 0.67x WH, WF 1.44x HE, DAO 0.12x WF, OOL 1.83x WOT, posterior ocellus distant from vertex crest $2.5 \mathrm{x}$ DAO. Vertex straight, with rounded corner. VOL 1.04x HE. Notaulus narrow, present on anterior third of mesoscutum. Propodeal disc $0.85 \mathrm{x}$ as wide as long, $0.68 \mathrm{x}$ as high as long, basal triangle not defined, median carina incomplete, but well-defined, posterior carina inconspicuous. Mesopleuron with polished callus. Fore wing with discoidal vein somewhat pigmented basally, interstitial with median vein. Genitalia: paramere with ventral arm 2.0x wider than dorsal arm; aedeagus mesoconvex, its apex not reaching apex of dorsal arm of paramere.

\section{Pseudisobrachium breviceps Evans, 1969} Figs 52-55

Holotype, male. ARGENTINA, Tucumán, El Solidad, 11 km W Las Cejas, 15.I-14.II.1967, Malaise trap, L. Stange col. (FIML).

Descriptions. Male. Length $3.76 \mathrm{~mm}$. Dark castaneous. Mandible with three apical teeth, uppermost wide. Clypeus with subtrapezoidal median lobe, apical margin slightly convex, median carina bifurcated and inconspicuous apically. Antennal segment XI 2.0x as long as wide, pubescence subappressed, longer than half of diameter of basal flagellomeres, with some long erect setae outstanding pubescence, sensilla elongate. Eye long-haired. Frons coriaceous, punctures shallow. WH $1.22 \mathrm{x}$ LH, WF 0.67x WH, WF 1.71x HE, DAO 0.13x WF, OOL 1.12x WOT, posterior ocellus distant from vertex crest $0.5 \mathrm{x}$ DAO. Vertex straight, corner rounded. VOL $0.7 x$ HE. Temple strongly diverging anterad. Notaulus complete. Propodeal disc 0.96x as wide as long, $0.65 \mathrm{x}$ as high as long, basal triangle small, disc otherwise polished, median carina incomplete, posterior carina ill defined; declivity with arched rugulose. Mesopleuron with polished callus. Fore wing with discoidal vein pigmented basally, not interstitial with median vein. Genitalia: paramere with ventral arm $2.0 \mathrm{x}$ wider than dorsal; aedeagus mesoconvex, apex emarginated, aligned with apex of dorsal arm of paramere.

\section{Pseudisobrachium bruesi Evans, 1969 Figs $56-58$}

Holotype, male. CUBA, Soledad, Cienfuegos, I-II.1927, C. T. \& B. B. Brues col. (MCZH, \#31731).

Descriptions. Male. Length $3.33 \mathrm{~mm}$. Dark castaneous. Mandible broad apically, with four apical teeth, uppermost broad, upper two smaller than sublower. Clypeus with trapezoidal median lobe, apical margin barely concave, median carina bifurcated apically. Antenna thick, segment XI $1.32 \mathrm{x}$ as long as wide, pubescence of basal flagellomeres subappressed, with some long erect setae outstanding pubescence. Frons somewhat strongly coriaceous, punctures shallow. WH 0.97x LH, WF 0.68x WH, WF 1.48x HE, DAO 0.13x WF, OOL 1.38x WOT, posterior ocellus distant from vertex crest 1.0x DAO. Vertex straight, corner rounded. VOL 1.2x HE. Temple parallel anteriorly. Notaulus short, present on anterior third of mesoscutum. Propodeal disc $0.89 \mathrm{x}$ as wide as long, $0.67 \mathrm{x}$ as high as long, 

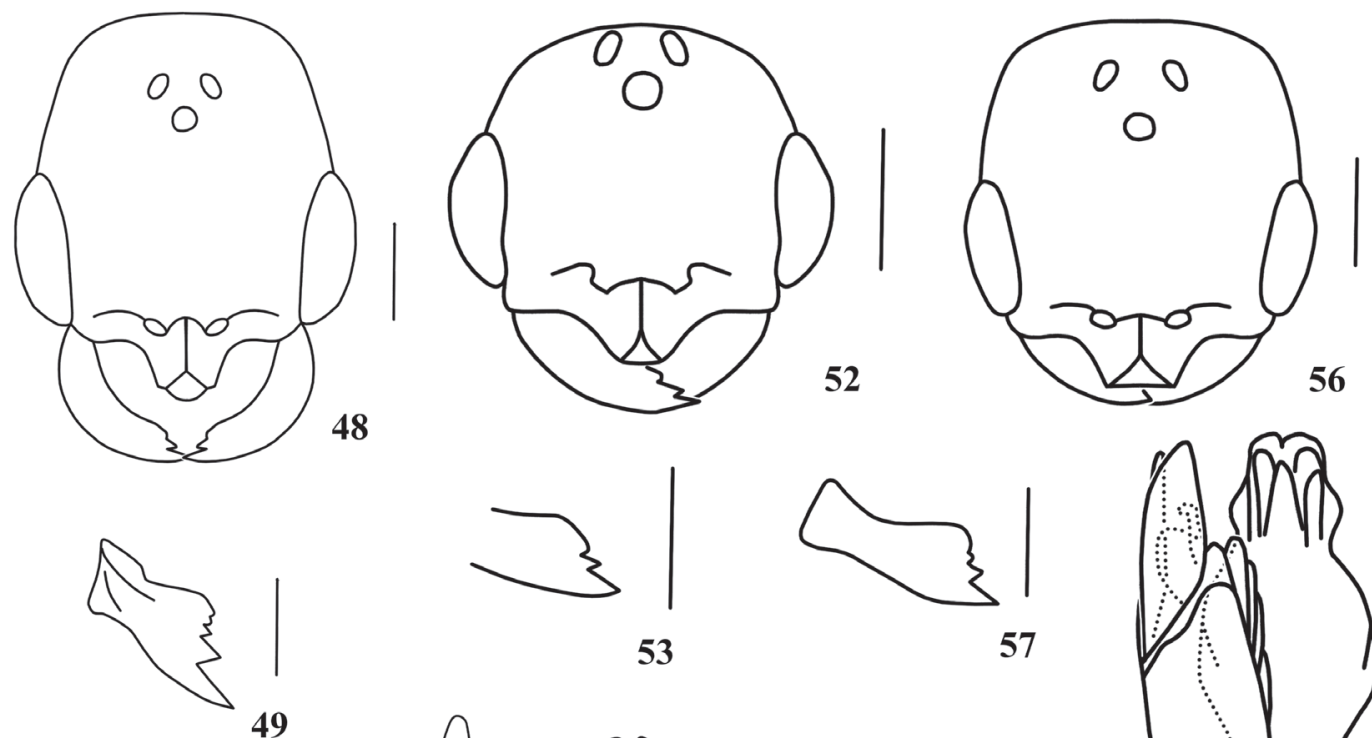

53

57
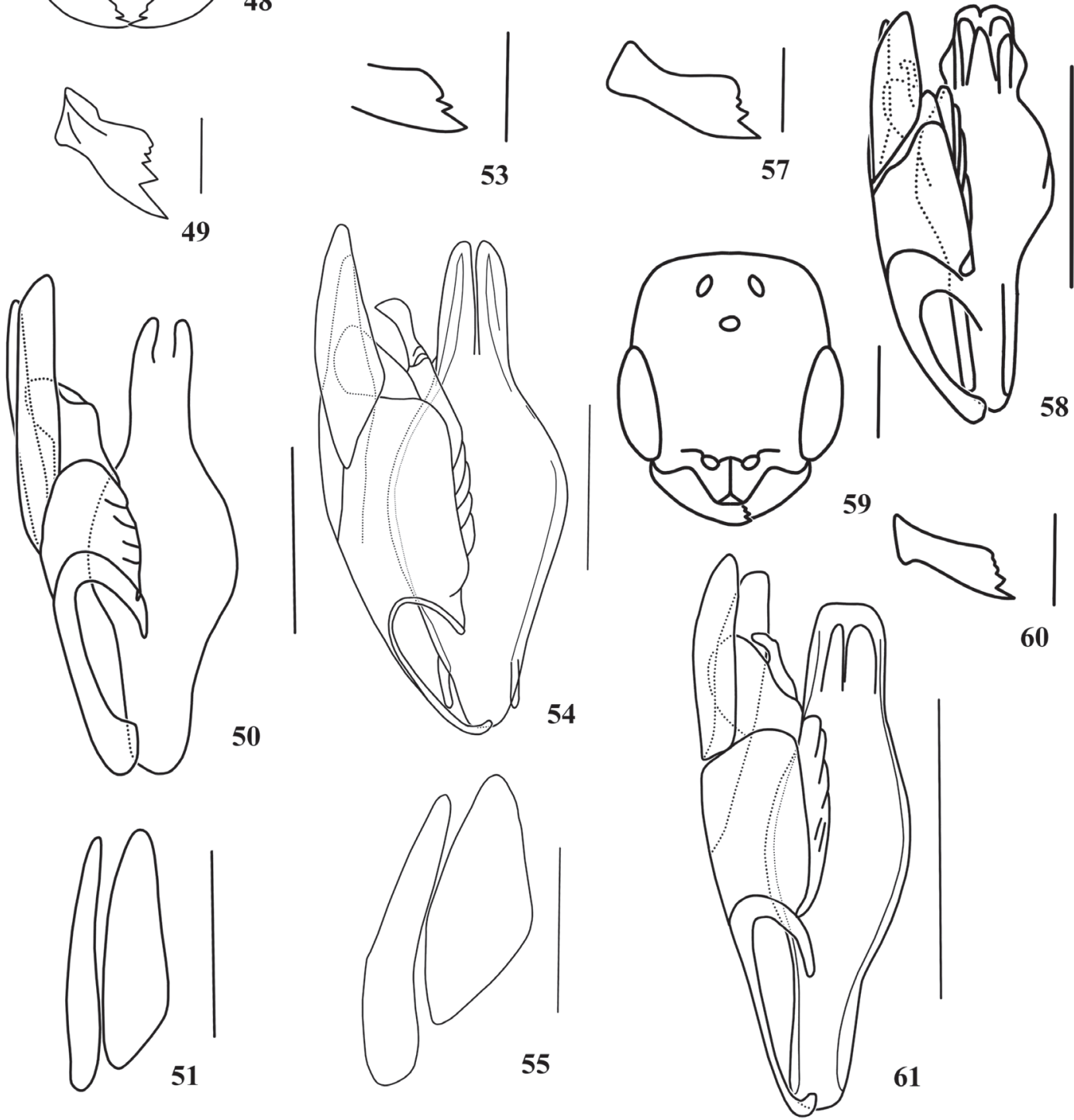

Figures 48-61. Pseudisobrachium brasiliense holotype male: (48) head, dorsal; (49) mandible, frontal; (50) genitalia, ventral; (51) paramere, full; P. breviceps holotype male: (52) head, dorsal; (53) mandible, frontal; (54) genitalia, ventral; (55) paramere, full; P. bruesi holotype male: (56) head, dorsal; (57) mandible, frontal; (58) genitalia, ventral; P. brunneum holotype male: (59) head, dorsal; (60) mandible, frontal; (61) genitalia, ventral. Bar $=250 \mu \mathrm{m}$. 
median carina incomplete, weakly rugulose, posterior carina absent. Mesopleuron with coriaceous callus. Fore wing with discoidal vein absent. Genitalia: paramere with ventral arm $1.8 \mathrm{x}$ wider than dorsal, dorsal arm slightly shorter; aedeagus mesoconvex, dilated below apex, its apex aligned with apex of dorsal arm of paramere, with two pairs of rounded lobes.

\section{Pseudisobrachium brunneum Evans, 1961 Figs 59-61}

Holotype, male. MEX[ICO], H[i]d[al]go, Zimapan, 1114.II.[19]51, at light, H. E. Evans col. (MCZH, \#30274).

Descriptions. Male. Length $3.02 \mathrm{~mm}$. Castaneous. Mandible with five apical teeth, upper three smaller than sublower. Clypeus with trapezoidal median lobe, median carina bifurcated apically. Antennal segment XI 1.33 as long as wide, pubescence of basal flagellomeres subappressed, with some long erect setae outstanding pubescence. Frons weakly coriaceous, punctures small. WH 0.92x LH, WF 0.61x WH, WF 1.27x HE, DAO 0.18x WF, OOL 1.11x WOT, posterior ocellus distant from vertex crest $1.2 x$ DAO. Vertex barely convex, corner rounded. VOL 0.81x HE. Temple parallel anteriorly. Notaulus present on anterior half of mesoscutum. Propodeal disc $0.77 \mathrm{x}$ as wide as long, $0.56 \mathrm{x}$ as high as long, median carina incomplete, weakly rugulose at basal triangle, posterior carina absent. Mesopleuron with weakly coriaceous callus. Fore wing with discoidal vein absent. Genitalia: paramere with ventral arm 2.0x wider than dorsal; aedeagus mesoconvex, apex wide, not reaching apex of dorsal arm of paramere.

\section{Pseudisobrachium burchellanum (Westwood, 1874)}

\section{Fig. 62}

Holotype, male. [BRAZIL], Brasilia, Canga, Corrego Raiz, nocte volitantia, XI.1828, Dom. Burchellio col. (OXUM, not found)

Descriptions. Male. Black. Mandible with five apical teeth, upper four of equal size. Propodeal disc about as wide as long, median carina distinct. Fore wing with discoidal vein pigmented, interstitial with median vein.

Remarks. I was not able to find the type. The information above and the illustration are extracted on the original publication.

\section{Pseudisobrachium calidum Evans, 1966} Figs 63-65

Holotype, male. COSTA RICA, Turrialba, rain forest, 26.VIII.[19]63, C. Porter col. (MCZH, \#31241).

Descriptions. Male. Length $4.92 \mathrm{~mm}$. Black. Mandible with five apical teeth, upper three teeth smaller than sublower. Clypeus with rounded median lobe, median carina bifurcated apically. Antenna thick, segment XI 1.7x as long as wide, pubescence of basal flagellomeres subappressed, with some long erect setae outstanding pubescence. Frons somewhat strongly coriaceous, punctures small. Head high in lateral view. WH 1.02x LH, WF 0.66x WH, WF 1.4x HE, DAO 0.14x WF, OOL 1.08x WOT, posterior ocellus distant from vertex crest $1.25 \mathrm{x}$ DAO. Vertex slightly convex, corner broadly rounded. VOL $0.68 x$ HE. Pronotal disc with strong and deep transverse foveolate groove paralleling posterior margin. Notaulus conspicuous, wide, foveolate, convergent posteriorly. Scutellar groove very wide. Propodeum very strongly rugulose, propodeal disc $1.0 \mathrm{x}$ as wide as long, $0.82 \mathrm{x}$ as high as long, median carina complete, posterior carina indistinguishable. Mesopleuron with weakly coriaceous callus. Fore wing with discoidal vein weakly pigmented basally, interstitial with median vein. Genitalia: paramere with ventral arm $2.0 \mathrm{x}$ wider than dorsal; aedeagus with sides concave, its apex much not reaching apex of dorsal arm of paramere.

Remarks. This species has the texture of the mesosoma unusually coarse.

\section{Pseudisobrachium capixabum Waichert \& Azevedo, 2004 Figs $66-69$}

Holotype, male. BRAZIL, E[spírito] S[anto], S[anta] Teresa, Est[ação] Biol[ógica de] Santa Lúcia, 26.IX.2001, sweeping, C.O. Azevedo \& R. Kawada col. (UFES).

Descriptions. Male. Length $3.4 \mathrm{~mm}$. Black; fore wing subhyaline. Mandible with five apical teeth, upper three smaller than sublower. Clypeus with trapezoidal median lobe, apical margin straight, median carina straight in profile. Antennal segment XI $1.17 \mathrm{x}$ as long as wide; pubescence of basal flagellomeres subappressed, with some erect setae outstanding pubescence. Frons strongly coriaceous, punctures shallow and inconspicuous. WH 0.98x LH, WF 0.67x WH, WF 1.37x HE; DAO 0.14x WF; OOL 1.38x WOT, posterior ocellus distant from vertex crest $0.78 x$ DAO. Vertex convex. VOL $0.85 x$ HE. Temple divergent anterad. Notaulus narrow, absent posteriorly. Propodeal disc $0.77 \mathrm{x}$ as wide as long, $0.5 \mathrm{x}$ as high as long, mostly rugulose, median carina incomplete, posterior carina absent; declivity strigulate; lateral of propodeum mostly weakly coriaceous. Mesopleuron with polished callus. Fore wing with discoidal vein weakly pigmented basally, nearly interstitial with median vein. Genitalia: paramere with ventral arm 3.0x wider than dorsal arm; aedeagus mesoconvex, its apex not reaching apex of dorsal arm of paramere.

\section{Pseudisobrachium castaneiceps Evans, 1966 Figs 70-71}

Holotype, male. BRAZIL, Santa Catarina, Nova Teutonia, 15.II.1961, F. Plaumann col. (MCZH, \#31213). Genitalia in slide HE\#629, lost.

Descriptions. Male. Length $6.09 \mathrm{~mm}$. Testaceous castaneous. Mandible broad apically, with five apical teeth. Clypeus with subtrapezoidal median lobe medial lobe, apical margin nearly straight, median carina bifurcated apically. Antennal segment slightly wider distally, segment XI 1.77 as long as wide, pubescence of basal flagellomeres subappressed, with 


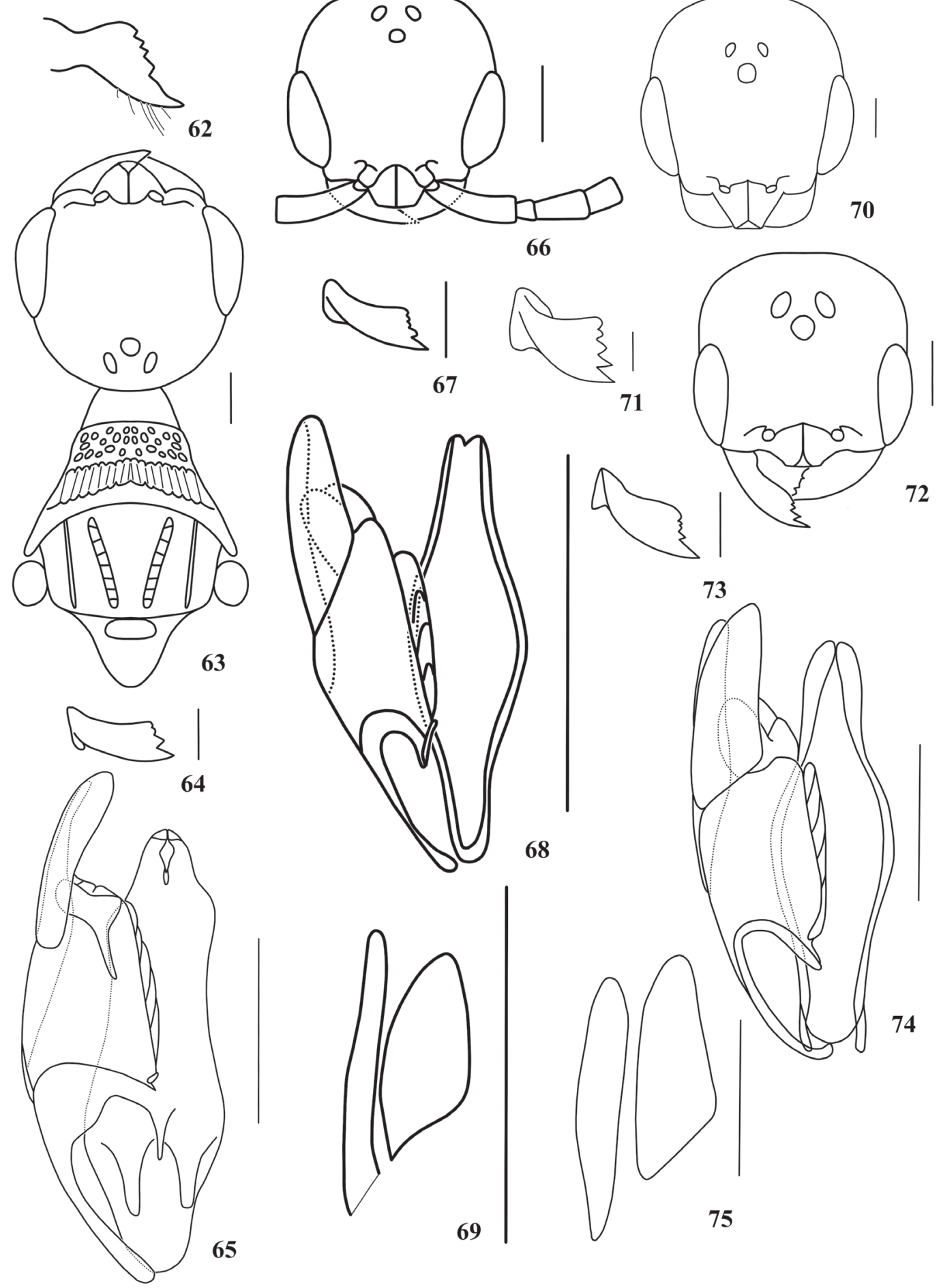

Figures 62-75. Pseudisobrachium burchellanum original illustration: (62) mandible, frontal; P. calidum holotype male: (63) head and anterior mesosoma, dorsal; (64) mandible, frontal; (65) genitalia, ventral; P. capixabum holotype male: (66) head, dorsal; (67) mandible, frontal; (68) genitalia, ventral; (69) paramere, full; P. castaneiceps holotype male: (70) head, dorsal; (71) mandible, frontal; P. chacoense holotype male: (72) head, dorsal; (73) mandible, frontal; (74) genitalia, ventral; (75) paramere, full. Bar $=250 \mu \mathrm{m}$. 
some erect setae outstanding pubescence. Eye densely hairy. Frons polished, punctures small. WH 0.92x LH, WF 0.64x WH, WF 1.34x HE, DAO 0.08x WF, ocellar triangle compact, OOL $1.39 \mathrm{x}$ WOT, posterior ocellus distant from vertex crest $2.5 \mathrm{x}$ $\mathrm{DAO}$, anterior margin of anterior ocellus reaching eye top imaginary line. Vertex slightly convex, corner rounded. VOL $0.81 \mathrm{x}$ HE. Notaulus conspicuous, convergent and incomplete posteriorly. Propodeal disc $1.0 \mathrm{x}$ as wide as long, $0.79 \mathrm{x}$ as high as long, median carina incomplete, triangle basal defined, with posterior median disc polished latero-posteriorly, posterior carina defined. Mesopleuron with polished callus. Fore wing with discoidal vein pigmented basally, interstitial with median vein.

Remarks. According to the original description, the aedeagus is entirely elliptical, extremely large and bulbous basally and terminating in a large membranous lobe.

\section{Pseudisobrachium chacoense Evans, 1973}

Figs $72-75$

Holotype, male. ARGENT[INA], Tucumán, El Solidad, 11 km W Las Cejas, 8-30.XII.1967, L. Stange col. (FIML).

Descriptions. Male. Length $5.0 \mathrm{~mm}$. Dark castaneous. Mandible with five apical teeth, uppermost broad, upper three teeth smaller than sublower. Clypeus with trapezoidal median lobe, apical margin nearly straight, median carina bifurcated just before apical margin. Antennal segment XI 1.85x as long as wide, pubescence subappressed, as long as half of diameter of basal flagellomeres, with some long erect setae outstanding pubescence. Eye long-haired. Frons somewhat strongly coriaceous, punctures shallow. WH 1.0x LH, WF 0.67x WH, WF 1.45x HE, DAO 0.17x WF, OOL 0.89x WOT, posterior ocellus distant from vertex crest 2.0x DAO, anterior margin of anterior ocellus nearly reaching eye top imaginary line. Vertex convex, corner rounded. VOL 0.9x HE. Temple parallel anteriorly. Notaulus short anteriorly. Propodeal disc $0.84 \mathrm{x}$ as wide as long, $0.55 \mathrm{x}$ as high as long, basal triangle small, disc polished, median carina complete, posterior carina absent. Mesopleuron with short callus. Fore wing with discoidal vein unpigmented basally, not interstitial with median vein. Genitalia: paramere with ventral arm 2.0x wider than dorsal; aedeagus mesoconvex, its apex not reaching apex of dorsal arm of paramere.

\section{Pseudisobrachium chilense Evans, 1969} Figs 76-79

Holotype, male. CHILE, Santiago, Queb. de la Plata, Rinconada Maipú, 33²1'S 7047’W, 510 m, 26.XII.1966, Malaise trap, L. Stange col. (FIML).

Descriptions. Male. Length $2.43 \mathrm{~mm}$. Dark castaneous. Mandible with four apical teeth, upper three small and nearly of equal size. Clypeus with trapezoidal median lobe, apical margin angularly concave, median carina bifurcated apically. Antennal segment XI 3.0x as long as wide, pubescence subappressed, as long as half of diameter of basal flagellomeres, with few erect setae outstanding pubescence. Frons weakly coriaceous, punctures small. WH 0.98x LH, WF 0.68x WH, WF 1.35x HE, DAO 0.18x WF, OOL 1.0x WOT, posterior ocellus distant from vertex crest 1.0x DAO. Vertex convex, corner broadly rounded. VOL $1.27 x$ HE. Notaulus absent. Propodeal disc $0.77 \mathrm{x}$ as wide as long, $0.69 \mathrm{x}$ as high as long, anterior half polished, posterior half polished, without median and posterior carinae. Mesopleuron with small callus. Fore wing with discoidal vein tubular, interstitial with median vein. Genitalia: paramere with ventral arm 2.0x wider than dorsal; aedeagus very narrow, side nearly straight, its apex reaching beyond apex of dorsal arm of paramere.

\section{Pseudisobrachium clypeatum Evans, 1961 Figs 80-83}

Holotype, male. [PANAMA, Panama, Canal Zone, Barro Colorado Island, 4.I.1929], D. Rockefeller Exp. Gertsch, C. H. Curran col. (AMNH).

Descriptions. Male. Length $4.8 \mathrm{~mm}$. Head black, mesosoma dark castaneous; fore wing subhyaline. Mandible with five apical teeth, upper three teeth smaller than sublower. Clypeus with rectangular median lobe, lateral angles sharpened, median tooth small, rounded and broad. Antenna with some long setae outstanding short pubescence, segment XI 2.1x as long as wide, pubescence of basal flagellomeres subappressed, with some long suberect setae outstanding pubescence. Eye long-haired. Frons coriaceous, punctures shallow. WH 0.97x LH, WH LH, WF 0.64x WH, WF 1.27x HE, OOL 1.6x WOT, DAO 0.13x WF, frontal angle of ocellar triangle compact acute, posterior ocelli distant from crest vertex $1.85 \mathrm{x}$ DAO. Vertex slightly convex, corner rounded. VOL 0.9x HE. Mesoscutum with large shallow punctures. Notaulus absent on posterior half of mesoscutum. Propodeal disc $0.83 \mathrm{x}$ as wide as long, $0.7 \mathrm{x}$ as high as long, median carina incomplete, mostly strigulate, posterior carina indistinguishable from strigulation; spiracles elliptical, inclined, dorso-laterad; declivity strigulate; lateral of propodeum nearly polished. Mesopleuron with large shallow punctures and broad callus. Fore wing with subdiscoidal vein weakly pigmented, interstitial with median vein. Genitalia: paramere with ventral arm $2.2 \mathrm{x}$ wider than dorsal; aedeagus mesoconvex, its apex reaching beyond apex of dorsal arm of paramere, constricted subapically.

\section{Pseudisobrachium collinum (Ashmead, 1894) Figs 84-86}

Holotype, male. SAINT VINCENT W. I., H. H. Smith col. (USNM, \#2492)

Descriptions. Male. Length $2.12 \mathrm{~mm}$. Dark castaneous. Mandible with two lower large teeth, broad apically, upper three smaller than sublower. Clypeus with subtrapezoidal median lobe. Antennal segment XI 1.6 as long as wide, pubescence long, suberect, as long as diameter of basal flagellomeres, with some 


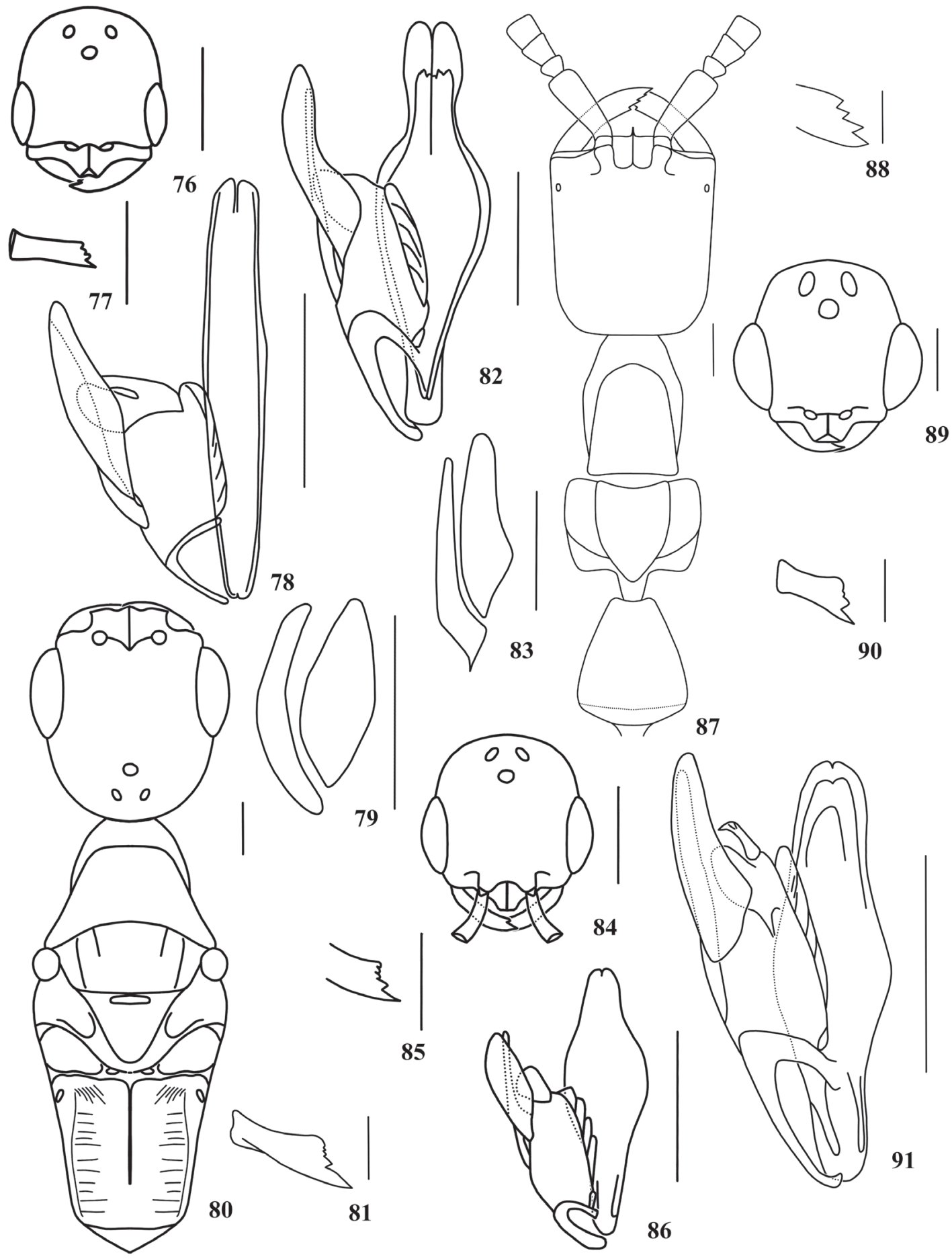

Figures 76-91. Pseudisobrachium chilense holotype male: (76) head, dorsal; (77) mandible, frontal; (78) genitalia, ventral; (79) paramere, full; P. clypeatum holotype male: (80) head and mesosoma, dorsal; (81) mandible, frontal; (82) genitalia, ventral; (83) paramere, full; $P$. collinum holotype male: (84) head, dorsal; (85) mandible, frontal; (86) genitalia, ventral; $P$. colombianum holotype female: (87) head and mesosoma, dorsal; (88) mandible, frontal; P. comanche holotype male: (89) head, dorsal; (90) mandible, frontal; (91) genitalia, ventral. Bar $=250 \mu \mathrm{m}$. 
long erect setae outstanding pubescence. Eye long-haired. Frons polished, punctures small and sparse. WH 1.0x LH; WF 0.68x WH, WF 1.38x HE, DAO 0.16x WF, OOL 1.41x WOT, posterior ocellus distant from vertex crest $0.86 x$ DAO. Vertex convex, corner broadly rounded. VOL 0.86x HE. Notaulus well impressed, convergent and nearly complete. Propodeal disc 0.69x as wide as long, $0.61 \mathrm{x}$ as high as long, median carina absent, strigulate, transverse posterior carina complete; declivity weakly rugulose. Mesopleuron with polished callus. Fore wing with discoidal vein nearly unpigmented basally, interstitial with median vein. Genitalia: paramere with ventral arm 3.0x wider than dorsal; aedeagus mesoconvex, its apex distinctly reaching beyond apex of dorsal arm of paramere.

Remarks. The original description was based on three specimens, but I was not able to see any other specimen at USNM other than examined here.

\section{Pseudisobrachium colombianum Vargas \& Terayama, 2002 Figs 87-88}

Holotype, female. COLOMBIA, Choco, Rio Sucio, Canal Maldito, 10m, 20.IX.1994, L. Mendoza col., VJIC 0133 (IAVHE 86698).

Descriptions. Female. Length $5.3 \mathrm{~mm}$. Dark castaneous. Mandible with four nearly of equal size apical teeth, upper two most smaller. Clypeus with trapezoidal median lobe, median carina high and straight in profile, extending beyond apical margin, apical margin triangular in frontal view. Eye light, elliptical, with one facet. Malar space about 2.0x as long as eye length. Frons polished, punctures conspicuous. Head with sides straight and subparallel, vertex straight, $1.2 \mathrm{x}$ as long as wide. Pronotal disc, mesoscutum and propodeal disc in ratio of 35:30:35. Mid tibia spinose. Metasoma not petiolate.

\section{Pseudisobrachium comanche Evans, 1961 Figs 89-91}

Holotype, male. [U.S.A.], Ariz[ona], Santa Cruz Co., Pena Blanca, 3950', 24.VIII.1959, at light, H. E. Evans col. (MCZ, \#30287).

Descriptions. Male. Length $3.58 \mathrm{~mm}$. Castaneous. Mandible with four apical teeth, uppermost broad, upper two smaller than sublower. Clypeus with trapezoidal median lobe, median carina bifurcated apically. Antennal segment XI 1.33 as long as wide, pubescence of basal flagellomeres subappressed, with some long erect setae outstanding pubescence. Eye long-haired, bulging. Frons coriaceous, punctures inconspicuous. WH $1.04 \mathrm{x}$ LH, WF 0.55x WH, WF 1.02x HE, ocelli large, DAO 0.26x WF, OOL $1.18 \mathrm{x}$ WOT, posterior ocellus distant from vertex crest $0.47 \mathrm{x}$ DAO, anterior margin of anterior ocellus nearly reaching eye top imaginary line. Vertex nearly straight, corner rounded. VOL 0.7x HE. Notaulus short. Propodeal disc $1.0 \mathrm{x}$ as wide as long, $0.65 \mathrm{x}$ as high as long, median carina incomplete, with short longitudinal striae anteriorly, disc otherwise pol- ished, posterior carina absent. Mesopleuron with weakly coriaceous callus. Fore wing with discoidal vein absent. Genitalia: paramere with ventral arm $2.3 \mathrm{x}$ wider than dorsal; aedeagus mesoconvex, apex rounded with small emargination, nearly aligned with apex of dorsal arm of paramere.

\section{Pseudisobrachium complanatum Evans, 1969 Figs 92-95}

Holotype, male. ARGENTINA, Tucumán, El Solidad, 11 km W Las Cejas, 13-27.V.1967, L. Stange col. (FIML).

Descriptions. Male. Length $4.36 \mathrm{~mm}$. Dark castaneous. Mandible with four apical teeth, upper three smaller than sublower. Clypeus with trapezoidal median lobe, apical margin straight, median carina bifurcated and inconspicuous apically. Antennal segment XI 1.3 as long as wide, pubescence of basal flagellomeres appressed, shorter than half of diameter of basal flagellomeres, with some long suberect setae outstanding pubescence. Eye long-haired. Frons coriaceous, punctures shallow. WH 0.91x LH, WF 0.61x WH, WF 1.11x HE, DAO 0.21x WF, OOL $0.77 \mathrm{x}$ WOT, posterior ocellus distant from vertex crest 0.95x DAO. Vertex straight, corner rounded. VOL 0.8x HE. Temple parallel anteriorly. Notaulus incomplete. Propodeal disc $1.0 \mathrm{x}$ as wide as long, $0.68 \mathrm{x}$ as high as long, anterior half polished, posterior half polished, median carina absent on posterior half of disc, posterior carina inconspicuous. Mesopleuron with small callus. Fore wing with discoidal vein unpigmented, interstitial with median vein. Genitalia: paramere with ventral arm 1.3x wider than wide dorsal arm; vannus without grooves; aedeagus mesoconvex, ventral margin with large lobe, its apex not reaching apex of dorsal arm of paramere, base long.

Remarks. This is the only known species of Pseudisobrachium with ungrooved vannus.

\section{Pseudisobrachium concinum Evans, 1969 Figs 96-99}

Holotype, male. ARGENT[INA], Tucumán, Horco Molle, San X\{J\}axier Mts., 4-12.I.1966, L. Stange col. (FIML).

Descriptions. Male. Length $4.5 \mathrm{~mm}$. Dark castaneous. Mandible with five apical teeth, uppermost broad, upper three smaller than sublower. Clypeus with trapezoidal median lobe, apical margin convex medially, median carina bifurcated apically. Antennal segment XI 2.0x as long as wide, pubescence subappressed, longer than half of diameter of basal flagellomeres, with some long suberect setae outstanding pubescence, sensilla elongate. Eye long-haired. Frons strongly coriaceous, punctures inconspicuous. WH 0.96x LH, WF 0.61x WH, WF 1.28x HE, DAO 0.22x WF, OOL $0.92 x$ WOT, posterior ocellus distant from vertex crest $1.2 \mathrm{x}$ $\mathrm{DAO}$, anterior margin of anterior ocellus nearly reaching eye top imaginary line. Vertex convex, corner broadly rounded. VOL 0.9x HE. Temple strongly diverging anterad. Notaulus short. Propodeal disc $0.91 \mathrm{x}$ as wide as long, $0.56 \mathrm{x}$ as high as long, basal triangle large, median carina complete, posterior carina ill de- 

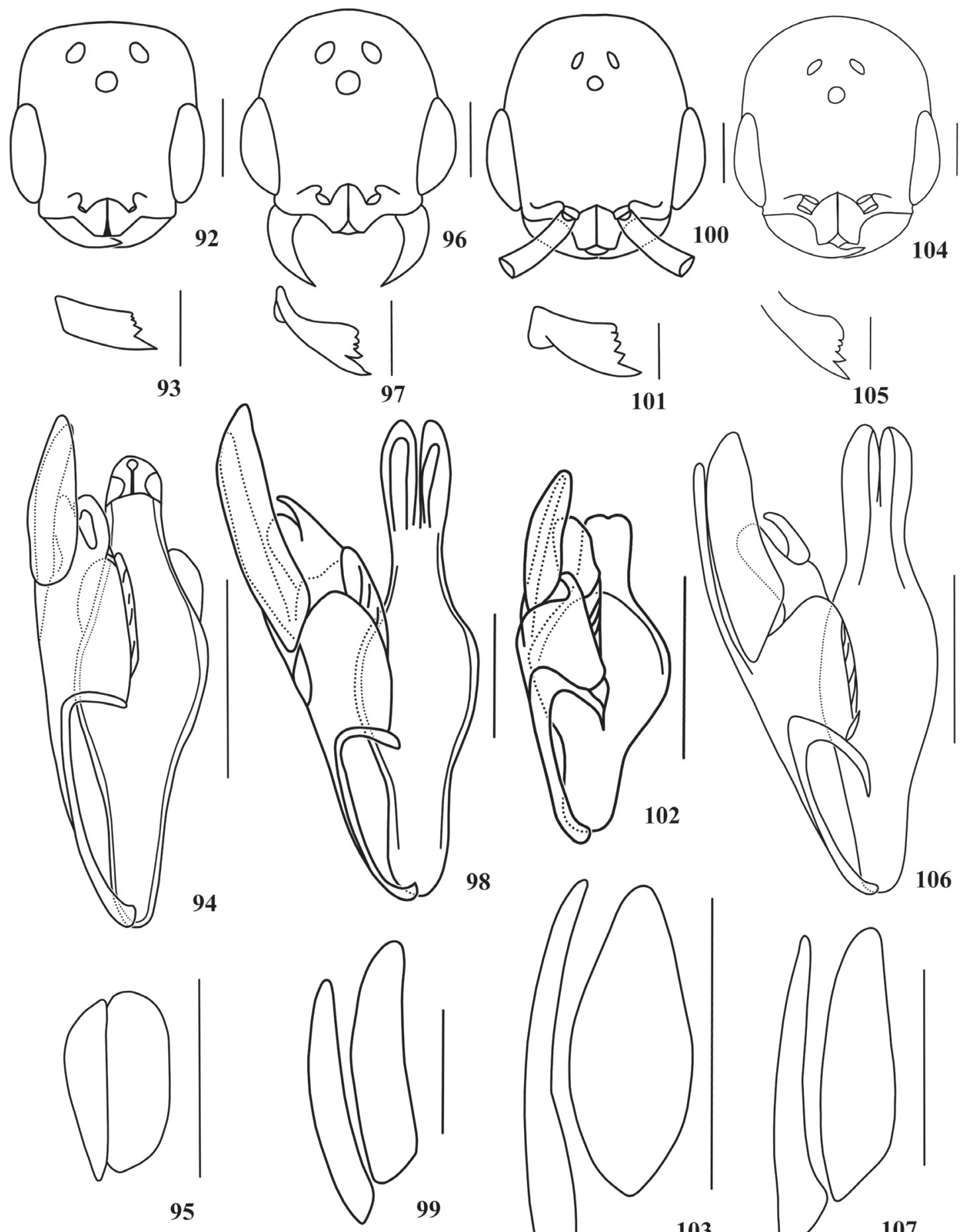

101

105

98
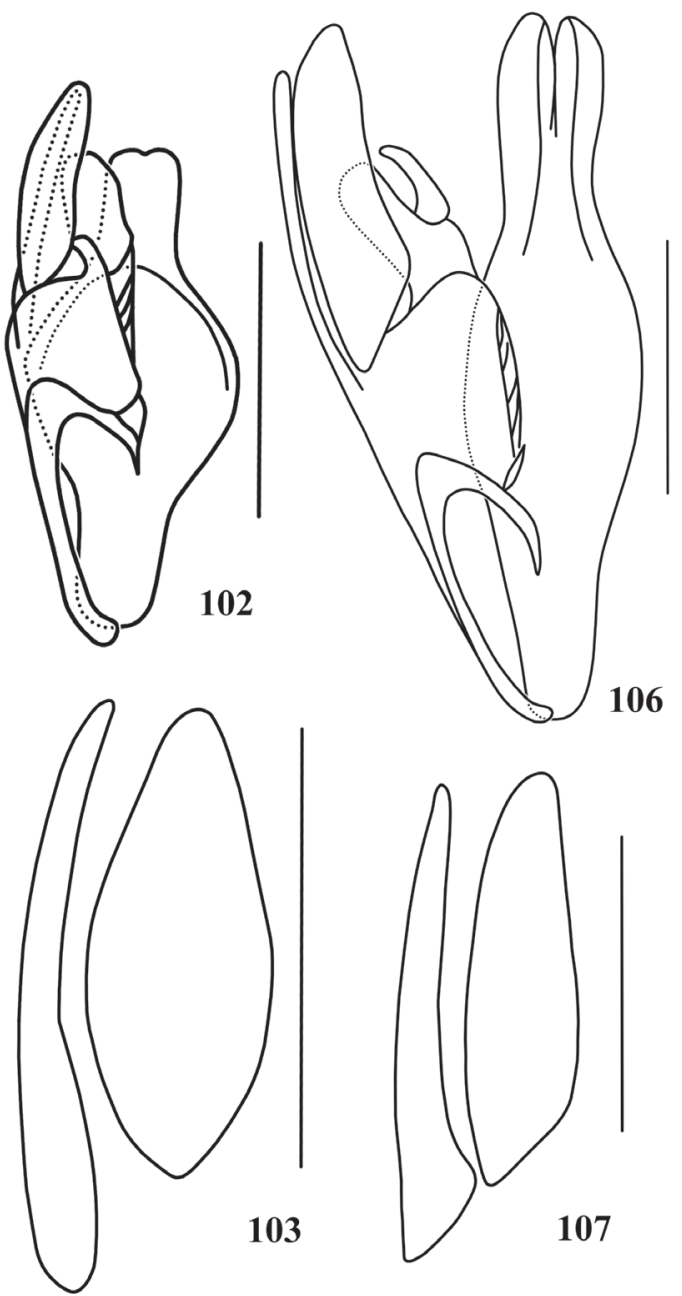

Figures 92-107. Pseudisobrachium complanatum holotype male: (92) head, dorsal; (93) mandible, frontal; (94) genitalia, ventral; (95) paramere, full; P. concinum holotype male: (96) head, dorsal; (97) mandible, frontal; (98) genitalia, ventral; (99) paramere, full; P. cooperi holotype male: (100) head, dorsal; (101) mandible, frontal; (102) genitalia, ventral; (103) paramere, full; P. corvinum holotype male: (104) head, dorsal; (105) mandible, frontal; (106) genitalia, ventral; (107) paramere, full. Bar $=250 \mu \mathrm{m}$. 
fined; declivity rugulose. Mesopleuron with polished callus. Fore wing with discoidal vein tubular for short distance, not interstitial with median vein. Genitalia: paramere with ventral arm $1.33 \mathrm{x}$ wider than dorsal; aedeagus mesoconvex, with subapical constriction, its apex not reaching apex of dorsal arm of paramere.

\section{Pseudisobrachium cooperi Evans, 1961 Figs 100-103}

Holotype, male. C[OSTA] R[ICA], Turrialba, 22.II.[19]49 (USNM, \#65152).

Descriptions. Male. Length $3.71 \mathrm{~mm}$. Black. Mandible broad apically, with five apical teeth, upper four nearly of equal size. Clypeus with trapezoidal median lobe, median tooth rounded, median carina bifurcated apically. Antennal segment XI 2.0 as long as wide, pubescence subappressed, about as long as half of diameter of basal flagellomeres, with some long erect setae outstanding pubescence. Eye long-haired. Frons coriaceous, punctures shallow. WH 0.99x LH, WF 0.64x WH, WF 1.45x HE, DAO $0.13 \mathrm{x}$ WF, ocellar triangle very compact, OOL 1.49x WOT, posterior ocellus distant from vertex crest $1.67 x$ DAO. Vertex slightly convex, corner broadly rounded. VOL $0.9 x$ HE. Notaulus on anterior half of mesoscutum. Propodeal disc $1.0 \mathrm{x}$ as wide as long, $0.76 \mathrm{x}$ as high as long, median carina incomplete, strongly strigulate, posterior carina indistinguishable, with pair of sublateral carinae incomplete anteriorly; declivity strigulate. Mesopleuron with polished callus. Fore wing with discoidal vein weakly pigmented basally, interstitial with median vein. Genitalia: paramere with ventral arm $3.1 \mathrm{x}$ wider than dorsal; aedeagus mesoconvex, swollen, its apex not reaching apex of dorsal arm of paramere.

\section{Pseudisobrachium corvinum Evans, 1969} Figs 104-107

Holotype, male. ARGENTINA, Tucumán, El Solidad, 11 km W Las Cejas, 15.I-14.II.1967, Malaise trap, L. Stange col. (FIML).

Descriptions. Male. Length $6.4 \mathrm{~mm}$. Black. Mandible with five apical teeth, uppermost broad, upper three smaller than sublower. Clypeus with trapezoidal median lobe, apical margin tridentate, median carina bifurcated apically. Antennal segment XI 1.7 $\mathrm{x}$ as long as wide, pubescence of basal flagellomeres appressed and short, with few long suberect setae outstanding pubescence. Eye long-haired. Frons polished, punctures conspicuous and dense. WH 0.95x LH, WF 0.69x WH, WF 1.5x HE, DAO 0.14x WF, OOL 1.06x WOT, posterior ocellus distant from vertex crest 2.2x DAO. Vertex convex, corner rounded. VOL 1.04x HE. Temple parallel anteriorly. Notaulus absent on posterior half of mesoscutum. Propodeal disc $0.91 \mathrm{x}$ as wide as long, $0.91 \mathrm{x}$ as high as long, entirely irregularly rugulose, median carina complete, posterior carina ill defined; declivity rugulose. Mesopleuron with polished large callus. Fore wing with discoidal vein weakly pigmented basally, not interstitial with median vein. Genitalia: paramere with ventral arm $1.8 \mathrm{x}$ wider than dorsal; aedeagus mesoconvex, little constricted subapically, apex aligned with apex of dorsal arm of paramere.

\section{Pseudisobrachium costaricanum Evans, 1961}

\section{Figs 108-109}

Holotype, female. COSTA RICA, S[an]ta Clara, Hamburg Farm, 30.IX.[19]26, F. Nevermann col. (USNM, \#65388).

Descriptions. Female. Length $3.97 \mathrm{~mm}$. Dark castaneous. Mandible with three of equal size apical teeth. Clypeus with apical margin concave and high, triangular in frontal view, margin with some bristles, median carina high and angularly produced beyond apical margin. Eye light, elliptical, with one facet. Malar space as long as eye length. Frons polished, densely punctuated. Head with sides straight and subparallel, vertex nearly straight, $1.25 \mathrm{x}$ as long as wide. Pronotal disc, mesoscutum and propodeal disc in ratio of 16:15:15. Mid tibia strongly spinose. Metasoma with short petiole.

\section{Pseudisobrachium coxalis (Cameron, 1888) Figs 110-115}

Holotype, male. PANAMA, Bugaba, Champion col., B. C. A. Hymen[optera] I. (BMNH, \#13233).

Descriptions. Male. Length $7.55 \mathrm{~mm}$. Black. Mandible with four large and nearly of equal size apical teeth. Clypeus with bidentate median lobe, apical margin concave, corner sharpened, median carina bifurcated apically. Antenna thick, pubescence of basal flagellomeres subappressed. Eye sparsely hairy. Frons polished, punctures conspicuous. WH 0.99x LH, WF 0.64x WH, WF 1.45x HE, DAO 0.1x WF, OOL 2.42x WOT, posterior ocellus distant from vertex crest 3.11x DAO, anterior margin of anterior ocellus reaching eye top imaginary line. Vertex rounded. VOL 0.79x HE. Notaulus well impressed, incomplete both anterior and posteriorly. Propodeal disc $1.18 \mathrm{x}$ as wide as long, $0.87 \mathrm{x}$ as high as long, median carina complete, posterior carina well-impressed. Mesopleuron with polished callus. Fore wing with discoidal vein almost entirely tubular, interstitial with median vein. Genitalia: paramere with ventral arm 2.0x wider than dorsal; aedeagus mesoconvex, base elongate, apex not reaching apex of dorsal arm of paramere, constricted below, ventral margin with large lobe.

\section{Pseudisobrachium crassicornis (Westwood, 1874) Figs 116-121}

Syntypes: male, [BRAZIL], Amazon[a]s, 1861, Bates col. (OXUM), here designated as lectotype, and male [BRAZIL], Amaz[onas] (OXUM), here designated as paralectotype.

Descriptions. Male. Length $8.08 \mathrm{~mm}$. Black. Mandible with five apical teeth, uppermost wide and blunt, subupper smaller, lower large. Clypeus with bidentate median lobe, apical margin concave, median carina bifurcated apicad. Antenna thick, segment XI 1.47 as long as wide, pubescence of basal flagellomeres subappressed, with many erected setae outstanding pubescence. Eye conspicuously hairy. Frons polished, densely punctuated. WH 0.92x LH, WF 0.63x WH, WF 1.27x HE, DAO 0.13x WF, OOL 1.32x WOT, posterior ocellus distant from vertex crest $2.86 \mathrm{x}$ 


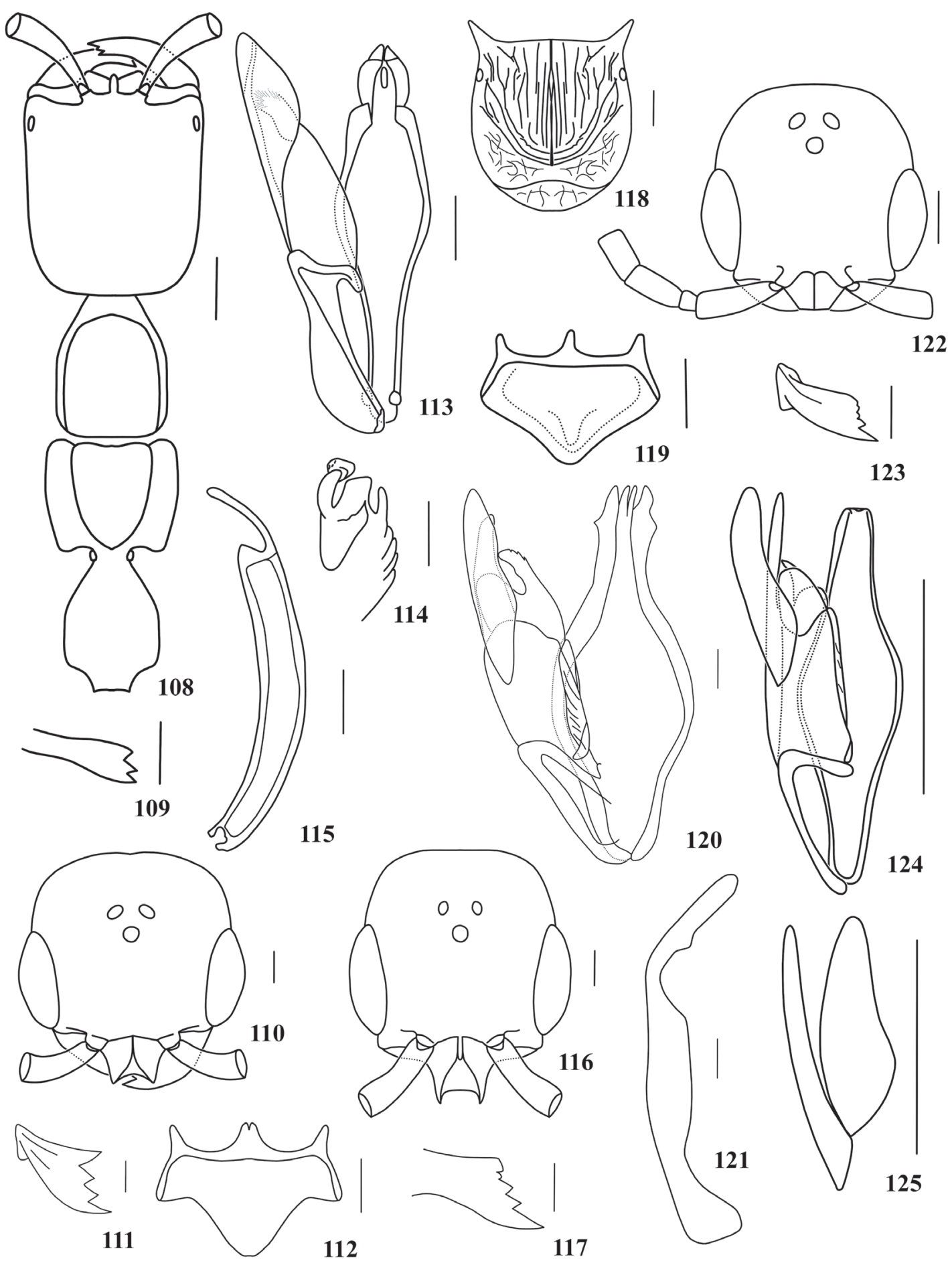

Figures 108-125. Pseudisobrachium costaricanum holotype male: (108) head and mesosoma, dorsal; (109) mandible, frontal; P. coxalis holotype male: (110) head, dorsal; (111) mandible, frontal; (112) hypopygium, ventral; (113) genitalia, ventral; (114) volsella, lateral; (115) aedeagus, lateral; P. crassicornis (Westwood, 1874), paralectotype male: (116) head, dorsal; (117) mandible, frontal; (118) propodeum, dorsal; (119) hypopygium, ventral; (120) genitalia, ventral; (121) aedeagus, lateral; P. cuspidatum holotype male: (122) head, dorsal; (123) mandible, frontal; (124) genitalia, ventral; (125) paramere, full. Bar $=250 \mu \mathrm{m}$. 
$\mathrm{DAO}$, posterior margin of anterior ocellus reaching eye top imaginary line. Vertex rounded. VOL 0.69x HE. Notaulus well impressed, incomplete anterior and posteriorly. Propodeal disc $1.25 \mathrm{x}$ as wide as long, $0.82 \mathrm{x}$ as high as long, median carina incomplete, bifurcated in posterior half, basal triangle only with longitudinal ridges, and surrounded by arched ridges, posterior carina indistinguishable. Mesopleuron with polished, large callus. Fore wing with discoidal vein weakly unpigmented basally, interstitial with median vein. Hypopygium with posterior margin subangulate. Genitalia: paramere with ventral arm $1.5 \mathrm{x}$ wider than dorsal, dorsal margin of dorsal arm angled medially; aedeagus mesoconvex, apex not reaching apex of dorsal arm of paramere inclined downward, outer margin denticulate, with two pairs of apical lobes, median one narrow.

\section{Pseudisobrachium cuspidatum Waichert \& Azevedo, 2004 Figs 122-125}

Holotype, male. BRAZIL, [Espírito Santo], S[anta] Teresa, Est[ação] Biol[ógica de] Santa Lúcia, 30.X.2001, sweeping, C.O. Azevedo col. (UFES).

Descriptions. Male. Length $5.1 \mathrm{~mm}$. Black; fore wing subhyaline. Mandible with five apical teeth, upper three smaller than sublower. Clypeus with trapezoidal median lobe, apical margin slightly convex medially. Antennal segment XI 2.0x as long as wide, pubescence of basal flagellomeres suberect, with some erect setae outstanding pubescence. Frons coriaceous, punctures shallow. WH 0.98x LH, WF 0.62x WH, WF 1.22x HE; DAO 0.14x WF; OOL 1.6x WOT, posterior ocellus distant from vertex crest $1.83 \mathrm{x}$ DAO. Vertex medially straight, corner broadly rounded. VOL $0.83 x$ HE. Notaulus well impressed, present on anterior fourth of mesoscutum. Propodeal disc $1.15 \mathrm{x}$ as wide as long, $0.73 \mathrm{x}$ as high as long, mostly rugulose, median carina almost complete, posterior carina indistinguishable from rugulation, declivity mostly strigulate, lateral of propodeum partially polished. Mesopleuron with polished callus. Fore wing with discoidal vein weakly pigmented basally, interstitial with median vein. Genitalia: paramere with ventral arm 3.0x wider than dorsal arm; aedeagus mesoconvex, its apex not reaching apex of dorsal arm of paramere.

Remarks. The metasoma is missing, so the measurement of body length was extracted from the original description.

\section{Pseudisobrachium dalmati Evans, 1961 Figs $126-130$}

Holotype, male. GUATEMALA, [Chimaltenango], [San Pedro] Yepocapa, [4850 feet], V.1948, H. T. Dalmat col. (USNM, \#65151).

Descriptions. Male. Length $5.37 \mathrm{~mm}$. Black. Mandible broad apically, with five apical teeth, upper three smaller than sublower. Clypeus with subtrapezoidal median lobe, apical margin convex, median carina bifurcated apically. Antenna thick, segment XI $1.4 \mathrm{x}$ as long as wide, pubescence of basal flagellomeres appressed, with some long erect setae outstanding pubescence. Eye long-haired. Frons polished, densely punc- tuated. WH 0.89x LH, WF 0.73x WH, WF 1.46x HE, DAO 0.13x WF, ocellar triangle compact, OOL 1.46x WOT, posterior ocellus distant from vertex crest $1.58 \mathrm{x}$ DAO. Vertex slightly convex, corner rounded. VOL $0.88 x$ HE. Notaulus on anterior half of mesoscutum. Propodeal disc $0.97 \mathrm{x}$ as wide as long, $0.5 \mathrm{x}$ as high as long, median carina incomplete, strigulate, posterior carina indistinguishable; declivity strigulate. Mesopleuron with polished callus. Fore wing with discoidal vein weakly pigmented basally, interstitial with median vein. Genitalia: paramere with ventral arm 1.6x wider than dorsal; cuspis subquadrate, aedeagus mesoconvex, with subapical constriction, its apex not reaching apex of dorsal arm of paramere.

\section{Pseudisobrachium demissum Evans, 1967 Figs 131-134}

Holotype, male. MEXICO, Sinaloa, 20 mi. Ethiopian Concordia, 3000 feet, 12.VIII.1964, W. R. M. Mason col. (CNCI, \#18353).

Descriptions. Male. Length $5.25 \mathrm{~mm}$. Dark castaneous, head black. Mandible with five apical teeth, upper three teeth smaller than sublower. Clypeus with rounded median lobe, median carina complete. Antennal segment XI 1.22 as long as wide, pubescence of basal flagellomeres subappressed, with some suberect setae outstanding pubescence. Frons coriaceous, punctures small. WH 0.95x LH, WF 0.68x WH, WF 1.37x HE, DAO 0.21x WF, OOL 0.77x WOT, posterior ocellus distant from vertex crest $0.72 x$ DAO. Vertex convex, corner broadly rounded. VOL $0.71 \mathrm{x}$ HE. Notaulus complete. Propodeal disc $1.0 \mathrm{x}$ as wide as long, $0.58 \mathrm{x}$ as high as long, median carina incomplete, posterior carina absent. Mesopleuron with polished callus. Fore wing with discoidal vein unpigmented basally, not interstitial with median vein. Genitalia: paramere with ventral arm 2.0x wider than dorsal arm; aedeagus mesoconvex, swollen, apex much not reaching apex of dorsal arm of paramere.

\section{Pseudisobrachium deplanatum Evans, 1969 Figs $135-138$}

Holotype, male. ARGENT[INA], Horco Molle, San X JJaxier Mts., 1-7.V.1966, L. Stange col. (FIML).

Descriptions. Male. Length $5.4 \mathrm{~mm}$. Black. Mandible with five apical teeth, uppermost broad, upper three teeth smaller than sublower. Clypeus with rectangular median lobe, corner sharpened, apical margin somewhat biconvex, median carina bifurcated apically. Antennal segment XI 2.3x as long as wide, pubescence subappressed, longer than half of diameter of basal flagellomeres, with some long erect setae outstanding pubescence, sensilla elongate. Eye long-haired. Frons weakly coriaceous, punctures shallow. WH 1.0x LH, WF 0.63x WH, WF 1.39x HE, DAO 0.17x WF, OOL 1.31x WOT, ocellar triangle compact, posterior ocellus distant from vertex crest 1.12x DAO. Vertex slightly convex, corner broadly rounded. VOL $0.83 x$ HE. Notaulus short anteriorly. Propodeal disc $0.93 \mathrm{x}$ as wide as long, $0.81 \mathrm{x}$ as high as long, basal triangle small, disc otherwise 


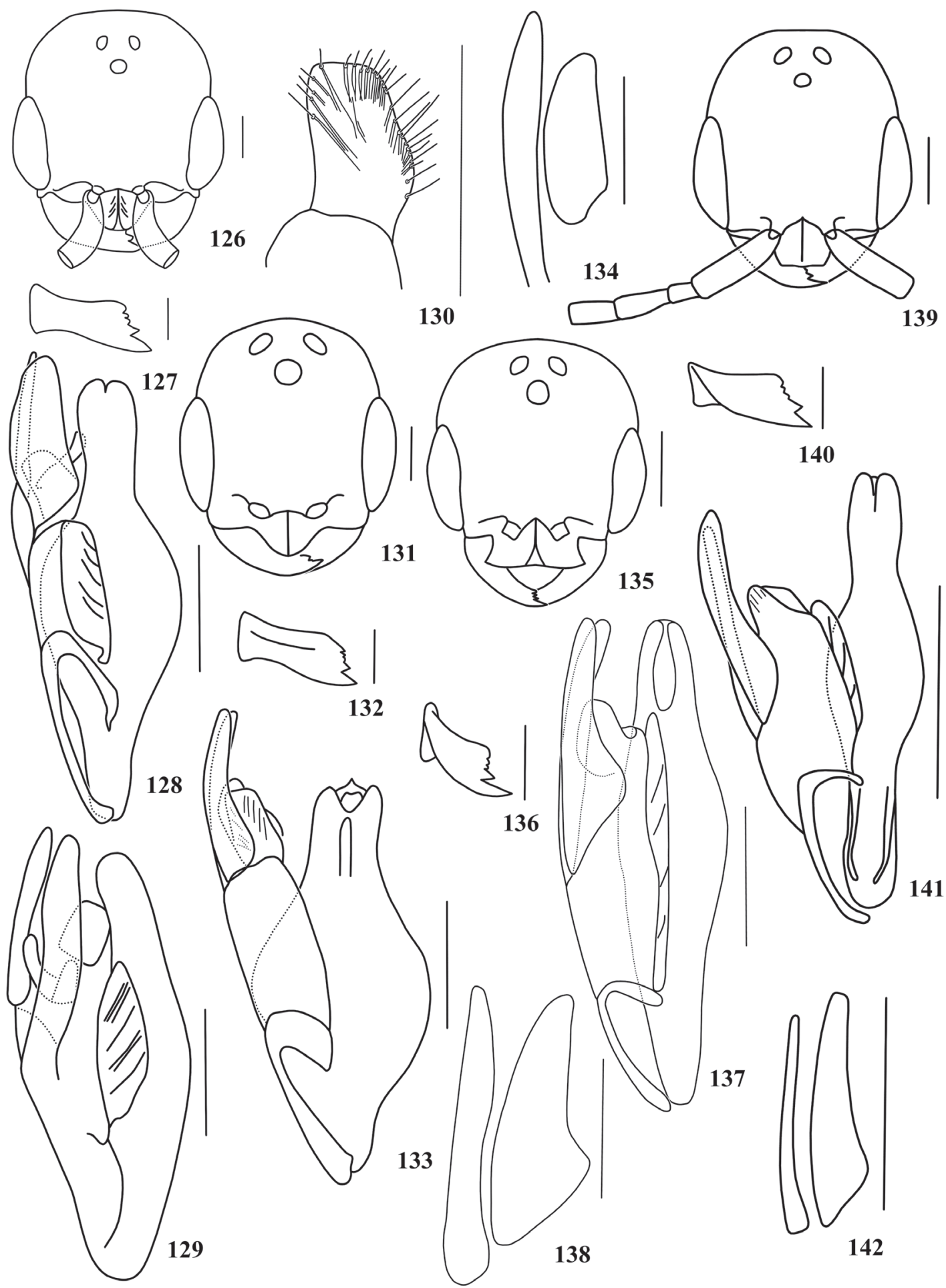

Figures 126-142. Pseudisobrachium dalmati holotype male: (126) head, dorsal; (127) mandible, frontal; (128) genitalia, ventral; (129) genitalia, lateral; (130) cuspis, full; P. demissum holotype male: (131) head, dorsal; (132) mandible, frontal; (133) genitalia, ventral; (134) paramere, full; P. deplanatum holotype male: (135) head, dorsal; (136) mandible, frontal; (137) genitalia, ventral; (138) paramere, full; P. distans holotype male: (139) head, dorsal; (140) mandible, frontal; (141) genitalia, ventral; (142) paramere, full. Bar = $250 \mu \mathrm{m}$. 
strigulate, median carina conspicuous, nearly complete, posterior carina indistinguishable; declivity strigulate. Mesopleuron with polished large callus. Fore wing with discoidal vein weakly pigmented basally, not interstitial with median vein. Genitalia: paramere with ventral arm $2.5 x$ wider than dorsal; aedeagus mesoconvex, apex wide and about aligned with apex of dorsal arm of paramere, base elongate.

\section{Pseudisobrachium distans (Kieffer, 1910) Figs 139-142}

Holotype, male. PERU, Cuzco, Cosnipata-Ebene, 1000m, 3.XII.1900, Garlep S.V.col. (ZMHB, \#201).

Descriptions. Male. Length $4.2 \mathrm{~mm}$. Dark castaneous, fore wing subhyaline. Mandible with five apical teeth, upper three smaller than sublower. Clypeus with trapezoidal median lobe, apical margin straight, median carina nearly complete. Antennal segment XI 2.2x as long as wide, pubescence of basal flagellomeres subappressed with some erect setae outstanding pubescence. Frons somewhat strongly coriaceous, punctures large and shallow. WH 0.86x LH, WF 0.69x WH, WF 1.42x HE, DAO 0.15x WF, OOL 2.2x WOT, posterior ocellus distant from vertex crest $1.36 \mathrm{x}$ DAO. Vertex badly convex, corner rounded. VOL $0.76 x$ HE. Notaulus absent on posterior half. Parapsidal furrow well-impressed, absent anteriorly. Propodeal disc $0.78 \mathrm{x}$ as wide as long, $0.58 \mathrm{x}$ as high as long, median carina nearly complete, posterior carina indistinguisable from strigulation, basal triangle large but weakly defined; declivity strigulate. Fore wing with discoidal vein weakly pigmented basally, not interstitial with median vein. Genitalia: paramere with ventral arm 3.0x wider than dorsal; aedeagus mesoconvex, with subapical constriction, its apex reaching beyond apex of dorsal arm of paramere.

\section{Pseudisobrachium distinguendum Kieffer, 1904 Figs 143-144}

Holotype, female. [PARAGUAY], Pto. 14 de Mayo, G. Boggiani col. (MCSN).

Descriptions. Female. Length $3.85 \mathrm{~mm}$. Head and mesosoma dark castaneous, metasoma castaneous. Mandible with four rounded apical teeth, upper three in oblique series. Clypeus with very wide trapezoidal median lobe, apical margin straight, triangular medially in frontal view, median carina somewhat high in profile. Eye light, subcircular, with one facet. Malar space slightly longer than eye length. Frons polished, densely punctuated with large punctures, with unpunctuated longitudinal median band. Anterior half of head with punctures in longitudinal channeled lines on dorsal, lateral and ventral sides. Head with sides nearly straight and subparallel, head slightly wider at malar level, vertex nearly straight, $1.26 \mathrm{x}$ as long as wide. Pronotal disc, mesoscutum and propodeal disc in ratio of 17:13:14. Mid tibia strongly spinose. Metasoma with short petiole.

\section{Pseudisobrachium elegantulum Ogloblin, 1925}

Holotype, male. BRAZIL: São Paulo, J. Mráz col. (NMPC, not found)

Descriptions. Male. Length $4.6 \mathrm{~mm}$. Head black, mesosoma dark castaneous with prothorax lighter; fore wing strongly subhyaline. Mandible with five apical teeth. Clypeus with trapezoidal median lobe. Antennal segment XI 1.86x as long as wide; pubescence of basal flagellomeres appressed. Frons coriaceous and punctuated. WH $0.89 \mathrm{x}$ LH, posterior ocellus distant from vertex crest $1.5 x$ DAO. VOL $0.82 x$ HE. Temple divergent anterad. Notaulus very short, anterior. Propodeal disc $0.8 \mathrm{x}$ as wide as long, basal triangle large, median carina incomplete, posterior carina absent. Fore wing with discoidal vein weakly pigmented, interstitial with median vein.

Remarks. The descriptions is extracted on the original publication.

\section{Pseudisobrachium erythrocephalum Evans, 1973} Figs 145-147

Pseudisobrachium cunco Perez, 1981. Syn. nov.

Holotype, male. CHILE, La Fusta, C. Lonquimay, Malleco, 14.II.1962, L. Ethiopian. Peña col. (CNCI, \#18352).

Descriptions. Male. Length $6.0 \mathrm{~mm}$. Black, head orange. Mandible with five large apical teeth, of equal size. Clypeus with trapezoidal median lobe, apical margin nearly straight, median carinae entirely duplicated, bifurcated apically. Antennal segment XI $1.67 \mathrm{x}$ as long as wide, pubescence of basal flagellomeres short, with some long erect setae outstanding pubescence. Eye small and hairy. Frons polished, punctures small. WH 0.93x LH, WF 0.71x WH, WF 2.0x HE, DAO 0.10x WF, OOL 1.83x WOT, posterior ocellus distant from vertex crest 3.57x DAO. Vertex rounded. VOL 1.48x HE. Pronotal disc short. Notaulus strong and complete. Propodeal disc reticulate, $0.95 \mathrm{x}$ as wide as long, $0.67 \mathrm{x}$ as high as long, median carina almost complete, posterior carina defined. Mesopleuron with polished and broad callus. Fore wing with discoidal vein weakly pigmented, not interstitial with median vein. Genitalia: paramere with ventral arm $2.2 x$ wider than dorsal arm; aedeagus oval, its apex not reaching apex of dorsal arm of paramere, base wide.

\section{Pseudisobrachium cunco Perez, 1981. Syn. nov. Figs $148-150$}

Holotype, male. CHILE, Isla de Chiloé, Dalcahue, 1020.II.1957, G. Barría col. (Collection Perez, not found).

Descriptions. Male. Length $6.25 \mathrm{~mm}$. Black, head orange. Mandible with five large apical teeth, of equal size. Clypeus with trapezoidal median lobe, median carina bifurcated. Pubescence of basal flagellomeres short, with erect setae outstanding pubescence. Frons punctuated. WH 1.14x LH, WF 0.73x WH, WF 2.0x HE, DAO 0.1x WF, OOL 1.82x WOT, posterior ocellus distant from vertex crest 3.0x DAO. Vertex convex, corner rounded. VOL $1.29 \mathrm{x}$ HE. Temples slightly bulging. Propodeal disc $0.71 \mathrm{x}$ as wide 


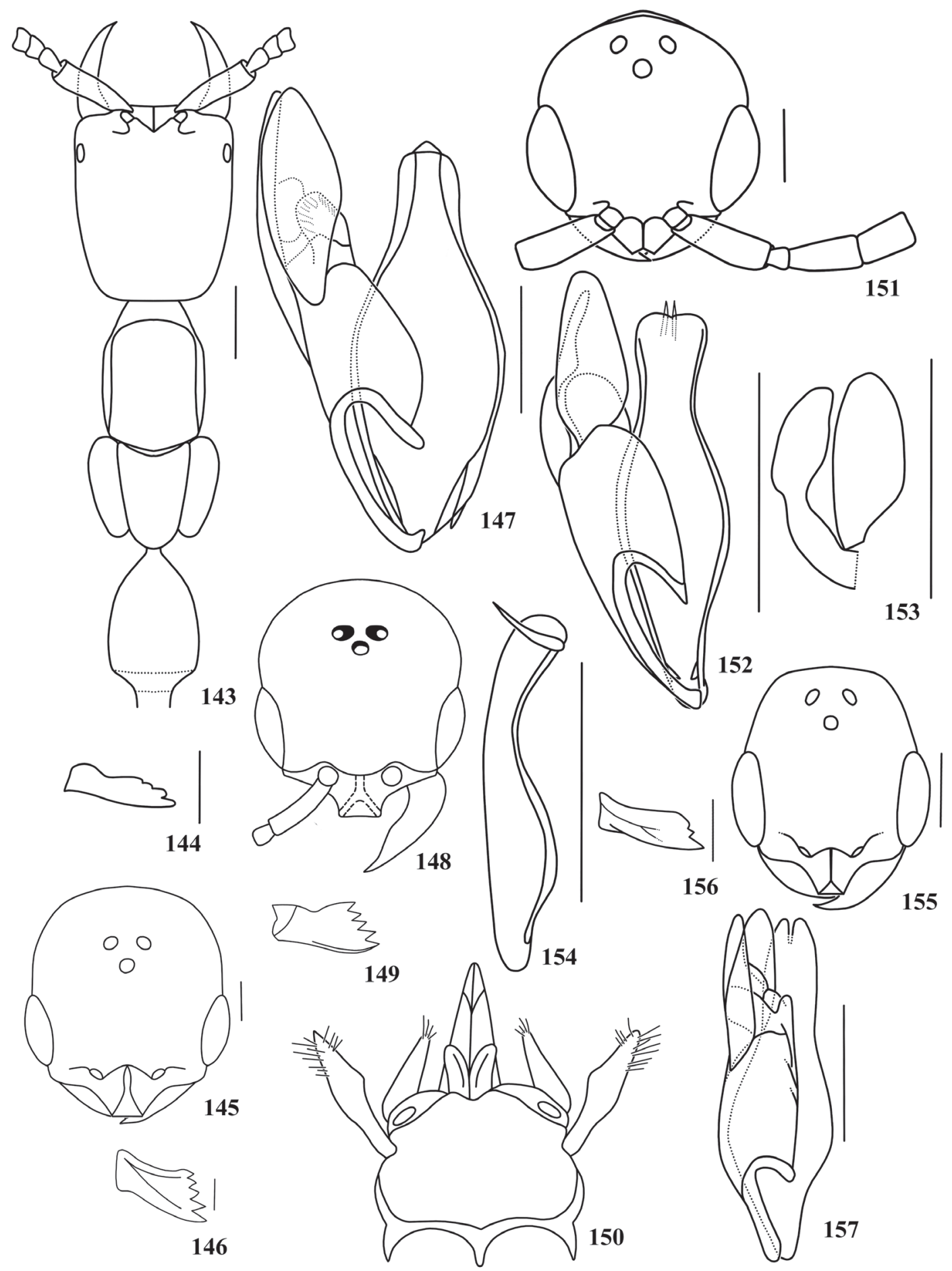

Figures 143-157. Pseudisobrachium distinguendum holotype female: (143) head and mesosoma, dorsal; (144) mandible, frontal; $P$. erythrocephalum holotype male: (145) head, dorsal; (146) mandible, frontal; (147) genitalia, ventral; P. cunco Perez, 1981 original illustration: (148) head, dorsal; (149) mandible, frontal; (150) genitalia and hypopygium, ventral; P. filum holotype male: (151) head, dorsal; (152) genitalia, ventral; (153) paramere, full; (154) aedeagus, lateral; P. flavicornis holotype male: (155) head, dorsal; (156) mandible, frontal; (157) genitalia, ventral. Bar $=250 \mu \mathrm{m}$. 
as long, $0.59 \mathrm{x}$ as high as long. Mesopleuron with callus. Fore wing with discoidal vein unpigmented, interstitial with median vein. Genitalia: paramere with ventral arm wider than dorsal.

Remarks. This species is proposed as new junior synonym of $P$. erythrocephalum Evans, by having the mandible with teeth of equal size, the eye very small, the head much developed behind the eye, and mainly the contrasting orange color of head in relation to the mesosoma. The illustration here presented was extracted from the original publication.

\section{Pseudisobrachium filum Waichert \& Azevedo, 2004 Figs 151-154}

Holotype, male. BRAZIL, E[spírito] S[anto], S[anta] Teresa, Est[ação] Biol[ógica de] Santa Lúcia, 23.VII.2001, sweeping, C.O. Azevedo \& R. Kawada col. (UFES).

Descriptions. Male. Length $3.6 \mathrm{~mm}$. Head black, mesosoma dark castaneous, prothorax lighter; fore wing subhyaline. Mandible with five apical teeth, upper three teeth smaller than sublower. Clypeus with trapezoidal median lobe, apical margin weakly angularly concave, lateral margin crenulated, median carina slightly arched. Antenna thick, segment XI 2.0x as long as wide; pubescence of basal flagellomeres subappressed, with some erect setae outstanding pubescence. Frons strongly coriaceous, punctures inconspicuous. WH 0.95x LH, WF 0.54x WH, WF 1.08x HE; DAO 0.19x WF; OOL 1.29x WOT, posterior ocellus distant from vertex crest $1.25 \mathrm{x}$ DAO. Vertex convex, occipital carina partially visible in dorsal view. VOL $0.82 x$ HE. Notaulus very short anteriorly. Propodeal disc $1.14 \mathrm{x}$ as wide as long, $0.91 \mathrm{x}$ as high as long, basal triangle small, disc otherwise areolate, median and posterior carinae absent, declivity areolate, lateral of propodeum coriaceous. Mesopleuron with coriaceous callus. Fore wing with discoidal vein unpigmented, not interstitial with median vein. Genitalia: paramere with ventral arm $1.4 \mathrm{x}$ wider than dorsal arm, dorsal arm unusually constricted medially; aedeagus mesoconvex, its apex not reaching apex of dorsal arm of paramere, with pair of filaments turned upward.

Remarks. The genitalia are in poor condition.

\section{Pseudisobrachium flavicornis (Kieffer, 1906) Figs $155-157$}

Holotype, male. NICARAGUA, Granada, Baker col. \#3487 (CASC, \#9644).

Descriptions. Male. Length $3.7 \mathrm{~mm}$. Dark castaneous. Mandible with three apical teeth, median one narrower. Clypeus with trapezoidal median lobe, median carina bifurcated apically. Antennal segment XI as long as wide, pubescence of basal flagellomeres subappressed, longer than half of diameter of basal flagellomeres, with some long erect setae outstanding pubescence. Frons coriaceous, punctures small. WH 0.97x LH, WF 0.67x WH, WF 1.33x HE, DAO 0.1x WF, OOL 1.24x WOT, posterior ocellus distant from vertex crest 2.0x DAO. Vertex barely convex, corner rounded. VOL 0.97x HE. Notaulus inconspicuous, present on anterior third of mesoscutum. Propodeal disc
$0.71 \mathrm{x}$ as wide as long, $0.71 \mathrm{x}$ as high as long, median carina nearly complete, posterior carina absent. Mesopleuron with polished and large callus. Fore wing with discoidal vein unpigmented, not interstitial with median vein. Genitalia: paramere with ventral arm 2.0x wider than dorsal arm; aedeagus mesoconvex, apex aligned with apex of dorsal arm of paramere.

\section{Pseudisobrachium flavinervis Fouts, 1928 Figs 158-161}

Pseudisobrachium foutsi Evans, 1961. Syn. nov. Pseudisobrachium emarginatum Evans, 1961. Syn. nov. Pseudisobrachium navajo Evans, 1961. Syn. nov. Pseudisobrachium rectangulatum Evans, 1961. Syn. nov.

Holotype, male. [U. S. A.], Cal[ifornia], Lone Star, 21.IX.1927, [take in soil] (USNM, \#41217).

Descriptions. Male. Length $4.33 \mathrm{~mm}$. Dark castaneous, head nearly black. Mandible with four apical teeth, upper two smaller than sublower. Clypeus with trapezoidal median lobe, median carina bifurcated apically. Antenna thick, segment XI $1.44 \mathrm{x}$ as long as wide, pubescence of basal flagellomeres appressed, with some long erect setae outstanding pubescence. Frons coriaceous, punctures shallow. WH 0.96x LH, WF 0.56x WH, WF 1.0x HE, ocelli large and bulging, DAO 0.32x WF, OOL $0.31 \mathrm{x}$ WOT, posterior ocellus distant from vertex crest $0.63 \mathrm{x}$ $\mathrm{DAO}$, posterior margin of anterior ocellus nearly reaching eye top imaginary line. Vertex slightly convex, corner rounded. VOL 0.56x HE. Pronotal disc depressed forward. Notaulus very inconspicuous, present on anterior half of mesoscutum. Propodeal disc $0.8 \mathrm{x}$ as wide as long, $0.44 \mathrm{x}$ as high as long, median carina incomplete, mostly areolate, posterior carina absent. Mesopleuron with coriaceous callus. Fore wing without discoidal vein. Genitalia: paramere with ventral arm 2.0x wider than dorsal; aedeagus mesoconvex, its apex not reaching apex of dorsal arm of paramere.

Remarks. Fouts (1928) indicate type and paratypes in the original description of this species, but apparently there are no correspondent labels attached to them. Because of this, I concluded that the specimen with the label "type" is the holotype, and did not designate lectotype for this species.

There are hundreds of specimens identified as $P$. flavinervis in the museums. The pronotal disc is depressed forward, the notauli are weak, and the propodeal disc is areolate. This species is highly variable, mainly in those characters associated to measurements of the head, especially the size of the ocelli. In average the ocelli are larger than those of other species in the genus.

\section{Pseudisobrachium emarginatum Evans, 1961. Syn. nov.}

\section{Figs 162-164}

Holotype, male. [U.S.A.], Texas, Kerrville, 10-14.VII.[19]53, light trap, L. J. Bottimer col. (USNM, \#65155).

Descriptions. Male. Length $3.5 \mathrm{~mm}$. Black. Mandible with four apical teeth, upper two smaller than sublower. Clypeus 

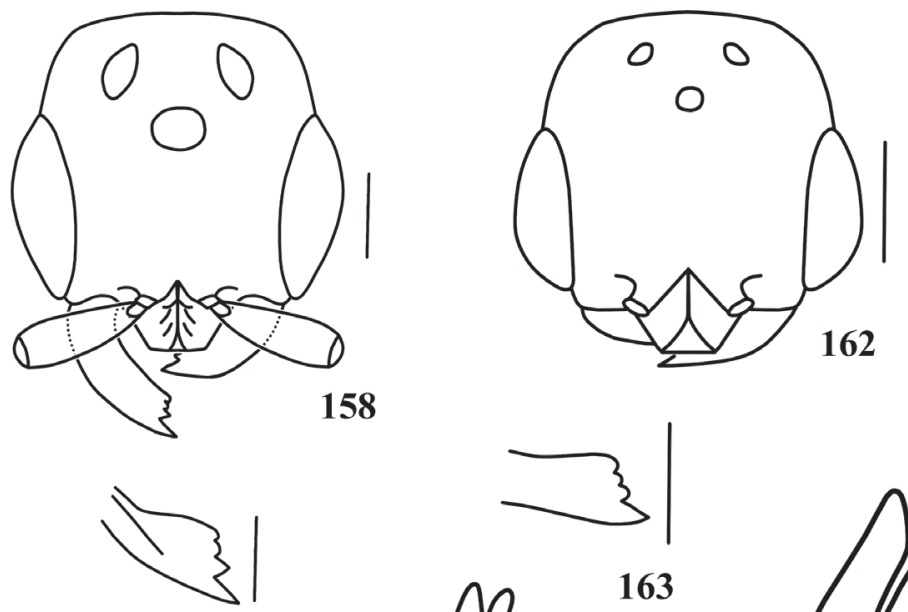

158

159
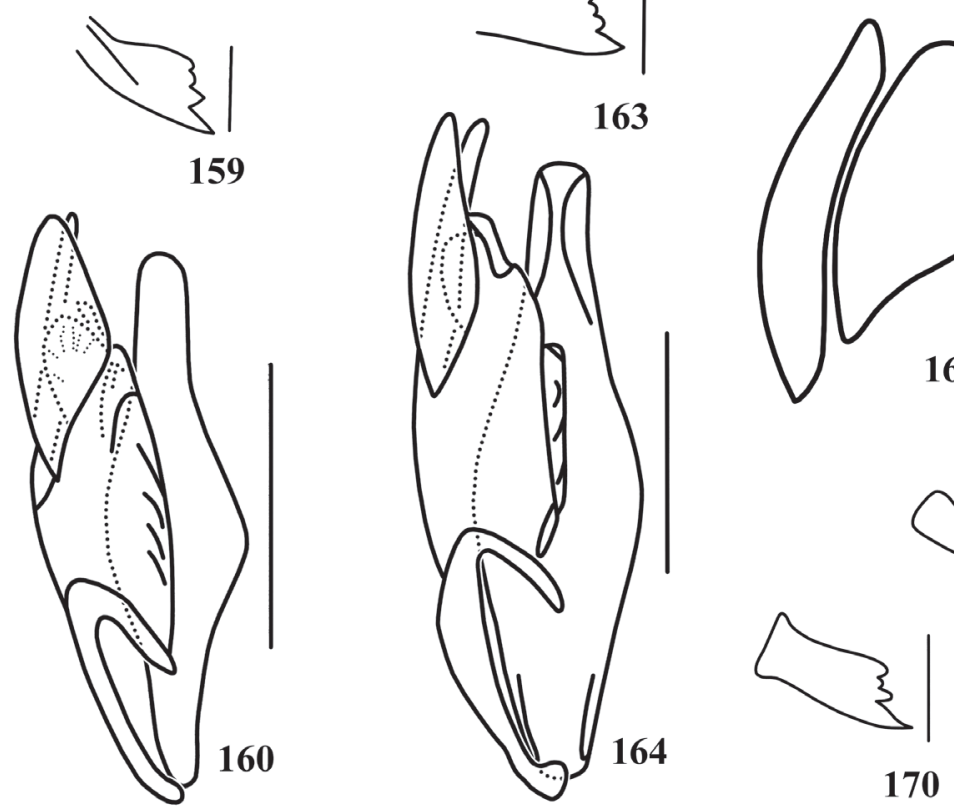

170

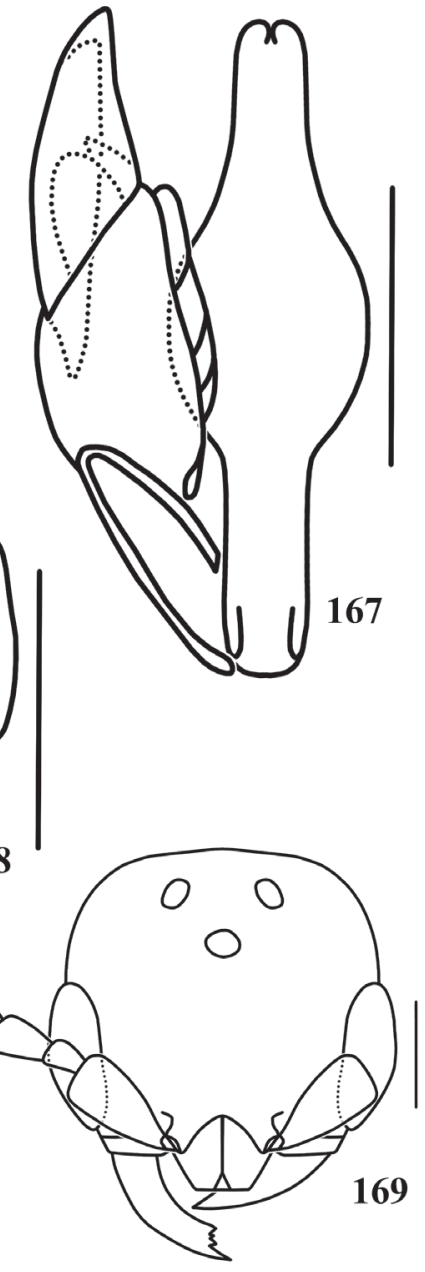

169

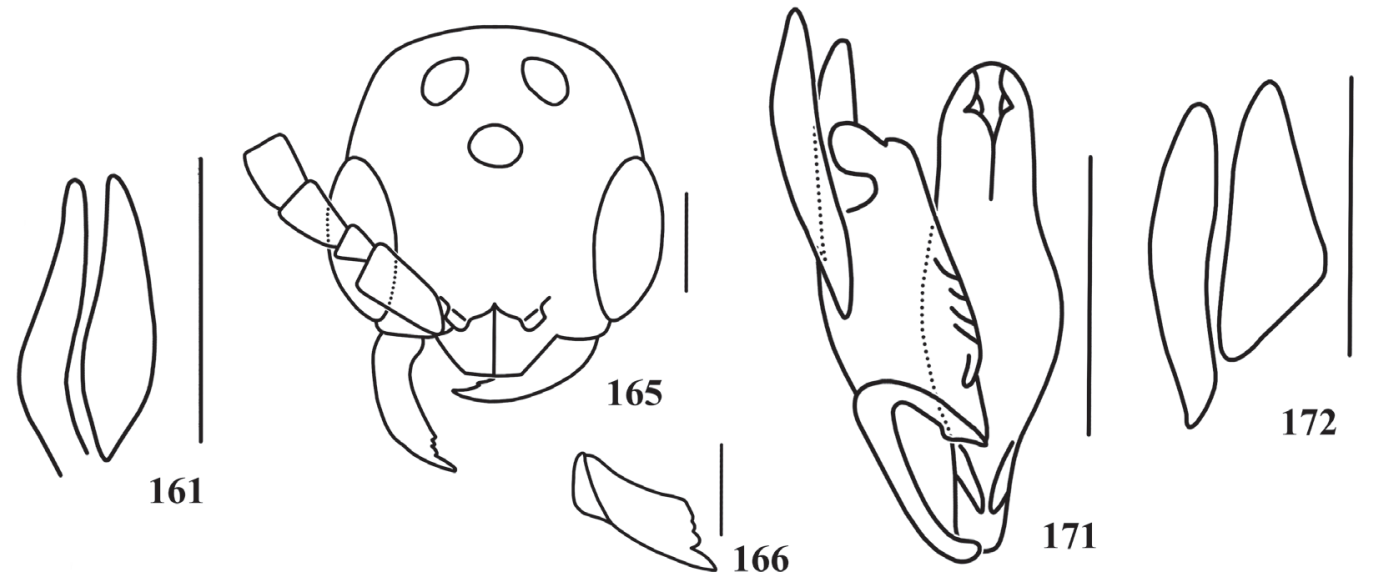

Figures 158-172. Pseudisobrachium flavinervis holotype male: (158) head, dorsal; (159) mandible, frontal; (160) genitalia, ventral; (161) paramere, full; P. emarginatum holotype male: (162) head, dorsal; (163) mandible, frontal; (164) genitalia, ventral; P. foutsi holotype male: (165) head, dorsal; (166) mandible, frontal; (167) genitalia, ventral; (168) paramere, full; P. navajo holotype male: (169) head, dorsal; (170) mandible, frontal; (171) genitalia, ventral; (172) paramere, full. Bar $=250 \mu \mathrm{m}$. 
with trapezoidal median lobe, median carina bifurcated apically. Antennal segment XI 1.43 as long as wide, pubescence subappressed, as long as half of diameter of basal flagellomeres, with some long suberect setae outstanding pubescence. Eye long-haired. Frons coriaceous, punctures shallow. WH 1.07x LH; WF 0.64x WH, WF 1.33x HE, DAO 0.18x WF, OOL 0.81x WOT, posterior ocellus distant from vertex crest $1.1 \mathrm{x}$ DAO. Vertex slightly convex, corner rounded. VOL 0.74x HE. Notaulus present on anterior third of mesoscutum. Parapsidal furrow complete. Propodeal disc $0.8 \mathrm{x}$ as wide as long, $0.67 \mathrm{x}$ as high as long, median carina conspicuous, incomplete, basal triangle areolate, posterior carina absent. Mesopleuron with weakly coriaceous callus. Fore wing without any trace of discoidal vein. Genitalia: paramere with ventral arm 3.0x wider than dorsal; aedeagus mesoconvex, its apex not reaching apex of dorsal arm of paramere, base wide.

Remarks. There are few specimens identified as $P$. emarginatum in museums throughout the world. They are characterized by having small ocelli. Evans (1978) associated to this name to $P$. foutsi. The general feature of the body and genitalia are similar to that of $P$. flavinervis Fouts and because of that I propose it as a new junior synonym for $P$. flavinervis.

\section{Pseudisobrachium foutsi Evans, 1961. Syn. nov. Figs 165-168}

Holotype, male. [U.S.A.], Texas, Cameron Co., P[or]t Isabel, 23-27.VI.1956, at light, H. E. Evans \& E. G. Mathews col. (MCZH, \#30289).

Descriptions. Male. Length $4.3 \mathrm{~mm}$. Dark castaneous, mesosoma lighter; fore wing hyaline. Mandible with four teeth, uppermost broad, upper two smaller than sublower. Clypeus with trapezoidal median lobe. Antenna with segment XI slightly longer than wide, pubescence of basal flagellomeres subappressed, with some long suberect setae outstanding pubescence. Eye long-haired. Frons coriaceous, punctures inconspicuous. WH 0.95x LH; WF 0.59x WH, WF 1.07x HE, ocelli large, DAO 0.3x WF, OOL 0.58x WOT, posterior ocellus distant from vertex crest $0.74 \mathrm{x} \mathrm{DAO}$, anterior margin of anterior ocellus reaching eye top imaginary line. Vertex straight, corner rounded. VOL 0.79x HE. Notaulus present on anterior third of mesoscutum. Propodeal disc $0.83 \mathrm{x}$ as wide as long, $0.7 \mathrm{x}$ as high as long, median carina incomplete, basal triangle ill defined, posterior carina absent; declivity and lateral of propodeum weakly coriaceous. Mesopleuron with coriaceous callus. Fore wing with discoidal vein unpigmented, interstitial with median vein. Genitalia: paramere with ventral arm $2.4 \mathrm{x}$ wider than dorsal; aedeagus mesoconvex, its apex reaching beyond apex of dorsal arm of paramere.

Remarks. There are many specimens identified as $P$. foutsi in museums throughout the world. They are characterized by having large ocelli. The general feature of the body and genitalia are similar to that of $P$. flavinervis Fouts and because of that I propose it as a new junior synonym for $P$. flavinervis.

\section{Pseudisobrachium navajo Evans, 1961. Syn. nov. Figs 169-172}

Holotype, male. [U.S.A.], Ariz[ona], Coconino Co., Tuba City, 27.VII.1954, [at light], H. E. \& M. A. Evans col. (MCZH, \#30282).

Descriptions. Male. Length $4.1 \mathrm{~mm}$. Dark castaneous, mesosoma lighter; fore wing hyaline. Mandible with four teeth, subupper smaller. Clypeus with trapezoidal median lobe, median carina high and angled in profile, bifurcated apically. Antenna with segment $\mathrm{X}$ slightly longer than wide, pubescence of basal flagellomeres appressed and short, with some long suberect setae outstanding pubescence. Eye long-haired. Frons somewhat strongly coriaceous, punctures shallow. WH 0.96x LH; WF 0.67x WH, WF 1.36x HE, DAO 0.16x WF, OOL 0.84x WOT, posterior ocellus distant from vertex crest $1.2 x$ DAO. Vertex slightly convex, corner rounded. VOL $0.9 x$ HE. Notaulus present on anterior half of mesoscutum. Propodeal disc $1.03 \mathrm{x}$ as wide as long, $0.66 \mathrm{x}$ as high as long, median carina complete, disc entirely rugulose, posterior carina absent; declivity aciculate; lateral of propodeum aciculate. Mesopleuron with polished callus. Fore wing with discoidal vein unpigmented, interstitial with median vein. Genitalia: paramere with ventral arm $1.8 \mathrm{x}$ wider than dorsal; aedeagus mesoconvex, apical third wide, apex rounded, not reaching apex of dorsal arm of paramere.

Remarks. There are few specimens identified as P. Navajo in museums throughout the world. They are characterized by having small ocelli. The general feature of the body and genitalia are similar to that of $P$. flavinervis Fouts and because of that I propose it as a new junior synonym for P. flavinervis. Only the holotype has the subupper mandibular teeth small (Fig. 170).

\section{Pseudisobrachium rectangulatum Evans, 1961. Syn. nov. Figs 173-176}

Holotype, male. [U.S.A.], Tex[as], Wilbarger Co., Red R[iver], 5.VII.1956, [at light], H. E. Evans \& E. G. Matthews col. (MCZH, \#30291).

Descriptions. Male. Length $3.1 \mathrm{~mm}$. castaneous. Mandible with four apical teeth, uppermost tooth wide, upper two smaller than sublower. Clypeus with trapezoidal median lobe. Antennal segment XI slightly longer than wide, pubescence of basal flagellomeres appressed and short, with some long erect setae outstanding pubescence. Eye long-haired. Frons coriaceous, punctures shallow. WH 0.93x LH; WF 0.62x WH, WF 1.17x HE, DAO 0.21x WF, OOL 0.61x WOT, posterior ocellus distant from vertex crest 1.0x DAO, anterior margin of anterior ocellus reaching eye top imaginary line. Vertex slightly convex, corner rounded. VOL 0.52x HE. Notaulus present on anterior half of mesoscutum. Parapsidal furrow complete. Propodeal disc 0.9x as wide as long, $0.57 \mathrm{x}$ as high as long, median carina incomplete, basal triangle large and rugulose, disc aciculate posteriorly, posterior carina absent; declivity and lateral of propodeum aciculate. Mesopleuron with undefined callus. Fore wing with- 


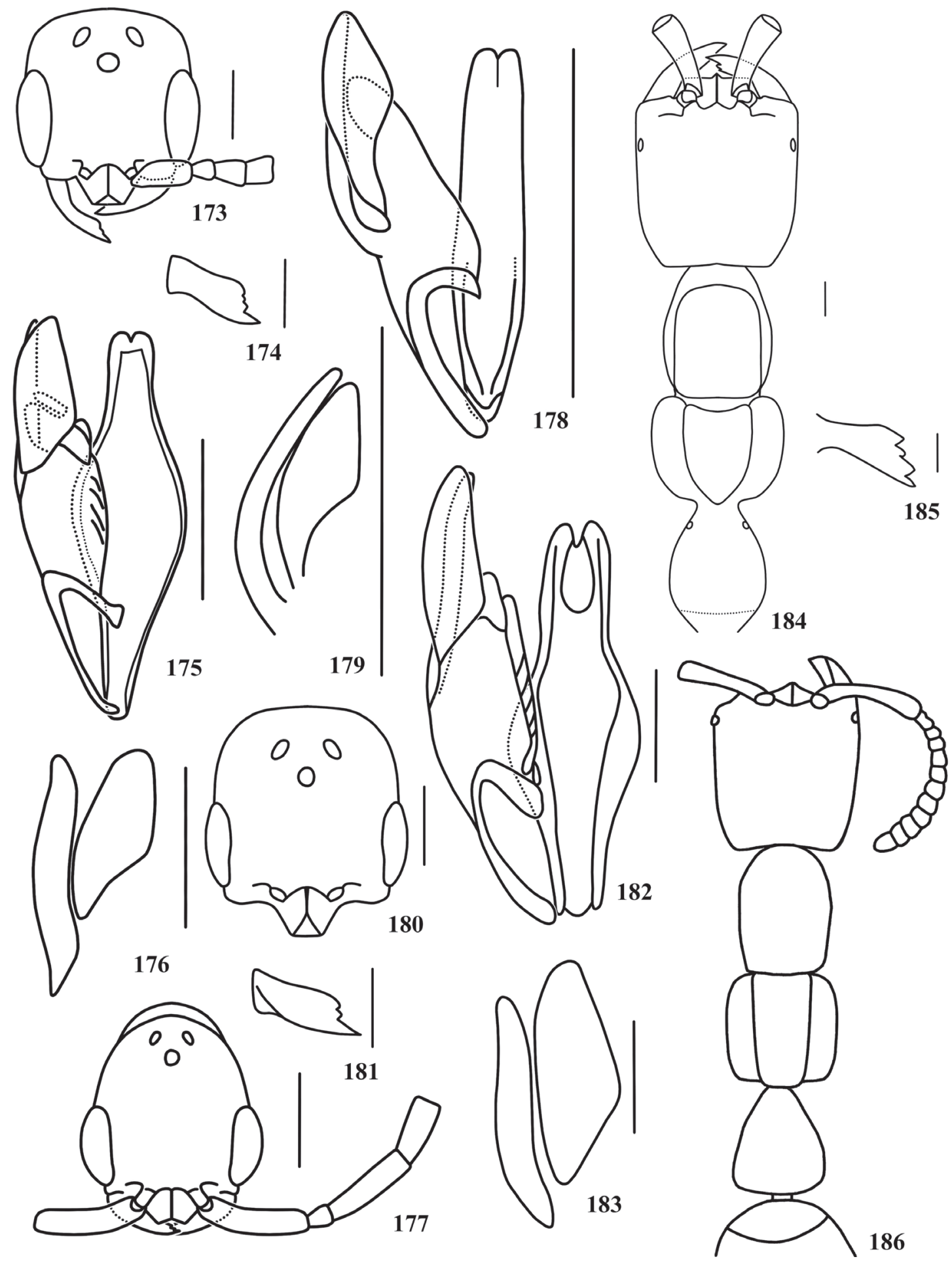

Figures 173-186. Pseudisobrachium rectangulatum holotype male: (173) head, dorsal; (174) mandible, frontal; (175) genitalia, ventral; (176) paramere, full; P. formosum holotype male: (177) head, dorsal; (178) genitalia, ventral; (179) paramere, full; P. fuscicornis holotype male: (180) head, dorsal; (181) mandible, frontal; (182) genitalia, ventral; (183) paramere, full; P. gigas holotype female: (184) head and mesosoma, dorsal; (185) mandible, frontal; P. graciliventre original illustration, female: (186) head and mesosoma, dorsal. Bar $=250 \mu \mathrm{m}$. 
out any trace of discoidal vein. Genitalia: paramere with ventral arm 1.9x wider than dorsal; aedeagus mesoconvex, its apex not reaching apex of dorsal arm of paramere.

Remarks. There are few specimens identified as $P$. rectangulatum in museums throughout the world. They are characterized by having small ocelli. Evans (1978) associated to this name to $P$. foutsi. The general feature of the body and genitalia are similar to that of $P$. flavinervis Fouts and because of that I propose it as a new junior synonym for $P$. flavinervis.

\section{Pseudisobrachium formosum Waichert \& Azevedo, 2004 Figs 177-179}

Holotype, male. BRAZIL, E[spírito] S[anto], S[anta] Teresa, Est[ação] Biol[ógica de] Santa Lúcia, 23.VII.2001, sweeping, C.O. Azevedo \& R. Kawada col. (UFES).

Descriptions. Male. Length $2.5 \mathrm{~mm}$. Head black, mesosoma dark castaneous, prothorax lighter; fore wing subhyaline. Mandible with five apical teeth, upper four small and nearly of equal size. Clypeus with trapezoidal median lobe, apical margin angularly concave, median carina high and angled in profile. Antennal segment XI $2.4 \mathrm{x}$ as long as wide; pubescence of basal flagellomeres subappressed, with some erect setae outstanding pubescence. Frons coriaceous, punctures small and sparse. WH 0.85x LH, WF 0.6x WH, WF 1.26x HE; OOL 1.75x WOT, posterior ocellus distant from vertex crest $1.67 \mathrm{x}$ DAO. Vertex convex, occipital carina strongly developed, well visible in dorsal view. VOL 1.15x HE. Temple divergent anterad. Notaulus absent. Propodeal disc $0.87 \mathrm{x}$ as wide as long, $0.65 \mathrm{x}$ as high as long; entirely areolate, median and posterior carinae absent; declivity and lateral of propodeum areolate. Mesopleuron with weakly coriaceous callus. Fore wing with discoidal vein weakly pigmented, interstitial with median vein. Genitalia: paramere with ventral arm 2.0x wider than dorsal arm, ventral arm wider medially, dorsal arm evenly arched; aedeagus narrow, sides nearly straight, its apex not reaching apex of dorsal arm of paramere.

Remarks. The genitalia are in poor condition.

\section{Pseudisobrachium fuscicornis Evans, 1969 Figs $180-183$}

Holotype, male. ARGENT[INA], Tucumán, Horco Molle, San X\{J\}axier Mts., 4-12.I.1966, L. Stange col. (FIML).

Descriptions. Male. Length $4.0 \mathrm{~mm}$. Black. Mandible with three apical teeth, upper two small and nearly of equal size. Clypeus with trapezoidal median lobe, apical margin straight, median carina bifurcated apically. Antennal segment XI as long as wide, pubescence appressed, as long as half of diameter of basal flagellomeres, with some erect setae outstanding pubescence. Eye long-haired. Frons coriaceous, punctures shallow. WH 0.89x LH, WF 0.71x WH, WF 1.53x HE, DAO 0.16x WF, OOL $1.14 \mathrm{x}$ WOT, posterior ocellus distant from vertex crest 1.5x DAO. Vertex slightly convex, corner rounded. VOL $1.16 \mathrm{x}$ HE. Temple parallel anteriorly. Notaulus short anteriorly. Propodeal disc $0.96 \mathrm{x}$ as wide as long, $0.74 \mathrm{x}$ as high as long, basal triangle large, median carina incomplete, posterior carina ill defined. Mesopleuron with polished large callus. Fore wing with discoidal vein unpigmented basally, interstitial with median vein. Genitalia: paramere with ventral arm $2.1 \mathrm{x}$ wider than dorsal; aedeagus mesoconvex, apex wide, rounded and emarginated, not reaching apex of dorsal arm of paramere.

Remarks. The holotype is lacking the metasoma, the following description of the genitalia and the respective illustration are based on one of the paratypes from the type-locality.

\section{Pseudisobrachium gigas Evans, 1961 Figs 184-185}

Holotype, female. [PANAMA], C[anal] Z[one], Barro Colo[rado], IX.1941, J. Zetek col. (USNM, \#65156).

Descriptions. Female. Length $6.4 \mathrm{~mm}$. Dark castaneous. Mandible with four apical teeth, subupper smaller. Clypeus with apical margin straight and high, triangular in frontal view, margin with some bristles, median carina high and angularly produced beyond apical margin. Eye light, subcircular, with one facet. Malar space longer than as eye length. Frons polished, densely punctuated. Head with sides mostly straight and nearly parallel, vertex nearly straight, $1.18 \mathrm{x}$ as long as wide. Pronotal disc, mesoscutum and propodeal disc in ratio of 25:20:24, with deep and wide suture separating pronotal disc and mesoscutum. Mid tibia and mid basitarsi strongly spinose. Metasoma with short petiole.

\section{Pseudisobrachium graciliventre Ogloblin, 1925}

\section{Fig. 186}

Holotype, female. BRAZIL: São Paulo, J. Mráz col. (NMPC, not found).

Descriptions. Female. Length $3.9 \mathrm{~mm}$. Black. Mandible with four apical teeth, two lowermost large. Clypeus with angulate median lobe. Eye elliptical. Frons shining, punctures dense and large. Head with sides nearly straight and subparallel, head wider at eye level, vertex slightly concave, $1.16 \mathrm{x}$ as long as wide. Pronotal disc, mesoscutum and propodeal disc in ratio of 19:17:16. Mid tibia spinose. Metasoma not petiolate.

Remarks. The descriptions and the illustration are extracted on the original publication.

\section{Pseudisobrachium haemorrhoidalis (Westwood, 1874) Figs 187-191}

Syntype male: Bres. [=Brazil], [Guerin-Meneville col.] (OXUM). Here designated as lectotype. [labels not original = first one: comunique par Guerin, second one: domaz moi le nord da ces hym. cumely soud aut. S. V. P.]

Descriptions. Male. Length $9.23 \mathrm{~mm}$. Black, apical half of metasoma castaneous, fore wing with yellowish transverse band at stigma level. Mandible wide apically, with five large of equal size apical teeth. Clypeus with trapezoidal median lobe, apical margin concave, median carina entirely bifurcated. Antenna slen- 
der, segment III 2.5 as long as wide, pubescence of basal flagellomeres suberect and long. Frons polished, regularly punctuated. WH 1.02x LH, WF 0.62x WH, WF 1.36x HE, DAO 0.14x WF, OOL $1.65 \mathrm{x}$ WOT, posterior ocellus distant from vertex crest $3.53 \mathrm{x}$ DAO, posterior margin of anterior ocellus reaching eye top imaginary line. Vertex convex. VOL $0.77 x$ HE. Pronotal disc with posterior half abruptly flat and polished delimited by ridge from anterior half. Parapsidal furrow well impressed and nearly complete. Scutellar groove wide. Propodeal disc $1.4 \mathrm{x}$ as wide as long, $0.84 \mathrm{x}$ as high as long, median carina conspicuous, but incomplete, disc strongly rugulose-rugulose posterior carina indistinguishable; declivity with arched ridges. Mesopleuron with polished, large callus, delimited by wide groove below. Fore wing with discoidal vein tubular basally, interstitial with median vein. Hypopygium with posterior margin deeply concave. Genitalia: paramere with ventral arm 2.0x wider than dorsal; aedeagus mesoconvex, neck thick, apex produced medially, reaching beyond apex of dorsal arm of paramere.

Remarks. The ocellar triangle is extremely compact, with the distance between the posterior ocelli much smaller than their longest diameter. The type is pinned through the mesoscutum so that it was not possible to describe the notauli.

Westwood (1874) studied another Brazilian specimen deposited at Royal Museum of Stockholm which I was not able to examine.

\section{Pseudisobrachium haywardi Ogloblin, 1950}

Fig. 192

Holotype, female: [ARGENTINA], Misiones, Puerto Iguazú, 30.I.1945, K. J. Hayward, A. Willink \& R. Golbach col. (FIML).

Descriptions. Female. Length $3.32 \mathrm{~mm}$. Dark castaneous. Mandible with four of equal size apical teeth. Clypeus with trapezoidal median lobe, apical margin concave, median carina not produced beyond apical margin. Frons polished. Head with sides straight and parallel, vertex slightly convex, $1.2 \mathrm{x}$ as long as wide. Pronotal disc, mesoscutum and propodeal disc in ratio of 16:14:16. Mid tibia strongly spinose. Metasoma with short petiole.

Remarks. The type is slide mounted. The material is strongly pressed, with the head crashed against the slide. The Canadian balsam is so dark to such an extent that is difficult to see the material. Because of that is hard to examine the punctures and the eyes, but according to the original description and the illustration, the eyes are very small, the malar space is several times longer than the eyes, the frons are densely punctuated with the median line without punctures.

\section{Pseudisobrachium howdeni Evans, 1969}

Figs 193-196

Holotype, male. JAMAICA, 4000 feet, Hardwar Gap, 29.VII.1966, Howden \& Becker col. (CNCI, \#10986).

Descriptions. Male. Length $5.0 \mathrm{~mm}$. Black. Mandible with five apical teeth, upper three smaller than sublower. Clypeus with trapezoidal median lobe, apical margin straight, median carina bifurcated apically. Antennal segment XI 2.5 as long as wide, pubescence of basal flagellomeres short, appressed, with some long suberect setae outstanding pubescence. Eye bulging. Frons coriaceous, punctures large. WH 0.98x LH, WF 0.71x WH, WF 1.6x HE, DAO 0.13x WF, OOL 1.5x WOT, posterior ocellus distant from vertex crest $1.13 \mathrm{x}$ DAO. Vertex rounded. VOL $1.15 \mathrm{x}$ HE. Notaulus virtually absent on posterior half of mesoscutum. Propodeal disc somewhat reticulate, as wide as long, $0.64 \mathrm{x}$ as high as long, median carina almost complete, posterior carina absent. Mesopleuron with coriaceous callus. Fore wing with discoidal vein somewhat pigmented basally, not interstitial with median vein. Genitalia: paramere with ventral arm $2.6 \mathrm{x}$ wider than dorsal arm; aedeagus mesoconvex, its apex aligned with apex of dorsal arm of paramere, base narrow.

\section{Pseudisobrachium hurdi Evans, 1961 Figs 197-198}

Holotype, male. MEXICO, Durango, 8 miles S. of Canutillo, 9.VIII.1951, P. D. Hurd col. (CASC, \#6869).

Descriptions. Male. Length $2.8 \mathrm{~mm}$. Dark castaneous. Mandible with five apical teeth, upper three very small and nearly fused. Clypeus with trapezoidal median lobe, median carina arched in profile, bifurcated apically. Antennal segment XI 1.1 as long as wide, pubescence of basal flagellomeres short and subappressed, with some erect setae outstanding pubescence. Eye small. Frons coriaceous, punctures small. WH 0.94x LH, WF 0.77x WH, WF 1.85x HE, DAO 0.12x WF, OOL $1.25 \mathrm{x}$ WOT, posterior ocellus distant from vertex crest 2.0x DAO. Vertex straight, corner rounded. VOL 1.3x HE. Temple parallel anteriorly. Notaulus present on anterior half of mesoscutum. Propodeal disc $0.88 \mathrm{x}$ as wide as long, $0.66 \mathrm{x}$ as high as long, median carina incomplete, posterior carina absent. Mesopleuron with weakly coriaceous callus, not much contrasting to remainder of pleuron. Fore wing with discoidal vein not pigmented, interstitial with median vein. Metasoma lost.

\section{Pseudisobrachium hypogeum Ogloblin, 1963 Figs 199-201}

Syntype female (three specimens): ARGENTINA, Buenos Aires, cerca de Bahía Blanca, salina Grünbein, 10.IV.1961, E. H. Rapoport col. (Ogloblin's collection, not found).

Descriptions. Female. Length $1.7 \mathrm{~mm}$. Light castaneous. Mandible with three apical teeth, lowermost large. Clypeus with rounded median lobe. Scape longer than mandible. Frons polished, punctures small. Head with sides slightly convex and subparallel, head wider at malar level, vertex slightly concave, $1.8 \mathrm{x}$ as long as wide. Subgenal carina present only on anterior third. Palpi with one segment. Occipital carina absent. Pronotal disc, mesoscutum and propodeal disc in ratio of 18:13:14. Pronotal disc with convex side. Mid tibia spinose. Metasoma with short petiole. 


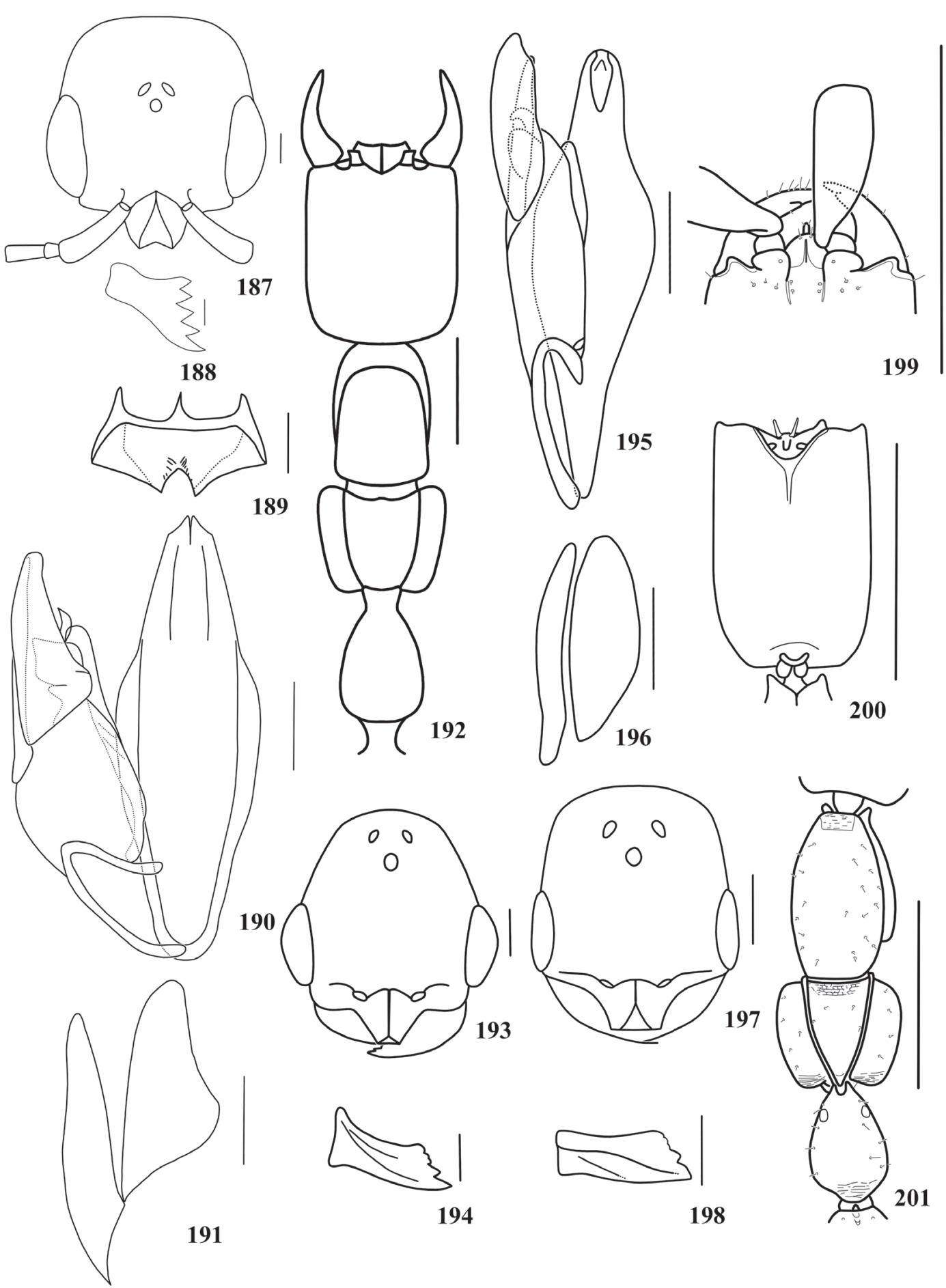

Figures 187-201. Pseudisobrachium haemorrhoidalis lectotype male: (187) head, dorsal; (188) mandible, frontal; (189)hypopygium, ventral. 190) genitalia, ventral; (191) paramere, full; P. haywardi holotype female: (192) head and mesosoma, dorsal; P. howdeni holotype male: (193) head, dorsal; (194) mandible, frontal; (195) genitalia, ventral; (196) paramere, full; $P$. hurdi holotype male: (197) head, dorsal; (198) mandible, frontal; P. hypogeum Ogloblin, 1963 original illustration, female: (199) anterior head, dorsal; (200) head ventral; (201) mesosoma, dorsal. Bar $=250 \mu \mathrm{m}$. 
Remarks. The descriptions and the illustration are extracted on the original publication. OgLoblin (1963) proposed the subgenus Edapholigon to accommodate this species mainly because it has the scape longer than the mandible, the subgenal carina short, the maxillary palpi with one segment and the occipital carina absent.

\section{Pseudisobrachium illippum Evans, 1969}

Figs 202-204

Holotype, male. ARGENTINA, Frias, Catamarca, 5.II.1951, Ros \& Michelbacher col. (CASC, \#11862).

Descriptions. Male. Length $3.8 \mathrm{~mm}$. Dark castaneous. Mandible with three apical teeth, upper two small and nearly of equal size. Clypeus with trapezoidal median lobe. Antennal segment XI 1.14 as long as wide, pubescence of basal flagellomeres somewhat short and appressed, with some erect setae outstanding pubescence. Frons coriaceous, punctures small. WH 1.0x LH, WF 0.61x WH, WF 0.96x HE, DAO 0.26x WF, OOL $0.42 x$ WOT, posterior ocellus distant from vertex crest 1.0x DAO, anterior margin of anterior ocellus reaching eye top imaginary line. Vertex slightly convex, corner rounded. VOL $0.46 \mathrm{x}$ HE. Notaulus present on anterior third of mesoscutum. Propodeal disc $0.86 \mathrm{x}$ as wide as long, $0.57 \mathrm{x}$ as high as long, median carina incomplete, posterior carina absent. Mesopleuron with slightly callus less coriaceous than remainder of pleuron. Fore wing with discoidal vein badly pigmented basally, interstitial with median vein. Genitalia: paramere with ventral arm 2.0x wider than dorsal arm; aedeagus mesoconvex, little dilated, its apex aligned with apex of dorsal arm of paramere.

\section{Pseudisobrachium inchoatum Kieffer, 1910}

Figs 205-208 \#9659).

Holotype, male. BRAZIL, Pará, Baker col., \#6741 (CASC,

Descriptions. Male. Length $4.9 \mathrm{~mm}$. Black. Mandible broad apically, with five apical teeth, uppermost wide, upper three smaller than sublower. Clypeus with trapezoidal median lobe, with large rounded median tooth, median carina bifurcated apically. Antennal segment XI 1.76 as long as wide, pubescence subappressed, longer than half of diameter of basal flagellomeres, with some long erect setae outstanding pubescence. Frons coriaceous, punctures conspicuous. WH 1.01x LH, WF 0.63x WH, WF 1.45x HE, DAO 0.11x WF, OOL 1.79x WOT, posterior ocellus distant from vertex crest $2.54 \mathrm{x}$ DAO, anterior margin of anterior ocellus closed to eye top imaginary line. Vertex convex, with rounded corner. VOL 0.81x HE. Thorax with large and closed punctures. Pronotal disc with transverse subapical depression. Notaulus present on anterior third of mesoscutum. Propodeal disc $0.95 \mathrm{x}$ as wide as long, $0.85 \mathrm{x}$ as high as long, median carina complete, posterior carina indistinguishable from rugulation. Mesopleuron with conspicuous and polished callus. Fore wing with discoidal vein unpigmented, interstitial with median vein. Genitalia: paramere with ventral arm 3.3x wider than dorsal arm; aedeagus mesoconvex, constricted subapically, its apex not reaching apex of dorsal arm of paramere, base wide.

\section{Pseudisobrachium intentum Waichert \& Azevedo, 2004} Figs 209-212

Holotype, male. BRAZIL, E[spírito] S[anto], Cariacica, Res[erva] Biol[ógica de] Duas Bocas, 22.X.1996, sweeping, E.H. Freitas col. (UFES).

Descriptions. Male. Length $5.5 \mathrm{~mm}$. Black, except dark castaneous posterior margin of pronotal disc; fore wing subhyaline. Mandible with five apical teeth, upper four nearly of equal size. Clypeus with trapezoidal median lobe, apical margin straight, median carina incomplete apically. Antennal segment XI 2.2x as long as wide; pubescence of basal flagellomeres subappressed, with some erect setae outstanding pubescence. Frons strongly coriaceous, punctures shallow. WH 0.99x LH, WF 0.66x WH, WF 1.31x HE; DAO 0.13x WF; OOL 1.43x WOT, posterior ocellus distant from vertex crest $1.48 \mathrm{x}$ DAO. Vertex medially. VOL $0.82 x$ HE. Temple divergent anterad. Notaulus present on anterior half of mesoscutum. Propodeal disc $0.94 \mathrm{x}$ as wide as long, $0.6 \mathrm{x}$ as high as long, basal triangle large, disc otherwise strigulate, median carina incomplete, posterior carina absent; declivity and lateral of propodeum mostly finely strigulate. Mesopleuron with nearly polished callus. Fore wing with discoidal veins weakly pigmented basally, nearly interstitial with median vein. Genitalia: paramere with ventral arm $2.0 x$ wider than dorsal arm; aedeagus mesoconvex, little dilated, apex wide, not reaching apex of dorsal arm of paramere, base elongate.

\section{Pseudisobrachium jamaicense Evans, 1969 Figs 213-216}

Holotype, male. JAMAICA, 4000 feet, Hardwar Gap, 25.VII.1966, Howden \& Becker col. (CNCI, \#10979).

Descriptions. Male. Length $5.0 \mathrm{~mm}$. Black. Mandible with five apical teeth, upper three smaller than sublower. Clypeus with trapezoidal median lobe, median carina bifurcated apically. Antennal segment XI 2.5 as long as wide, pubescence of basal flagellomeres subappressed, with some long erect setae outstanding pubescence. Frons strongly coriaceous, punctures small. WH 0.91x LH, WF 0.68x WH, WF 1.7x HE, DAO 0.14x WF, OOL 1.5x WOT, posterior ocellus distant from vertex crest 1.06x DAO. Vertex rounded. VOL 1.0x HE. Notaulus present on anterior half of mesoscutum. Propodeal disc reticulate, as wide as long, $0.59 \mathrm{x}$ as high as long, median carina almost complete, posterior carina absent. Mesopleuron with coriaceous callus. Fore wing with discoidal vein somewhat pigmented, not interstitial with median vein. Genitalia: paramere with ventral arm 2.5x wider than dorsal arm; aedeagus mesoconvex, swollen, its apex reaching beyond apex of dorsal arm of paramere. 


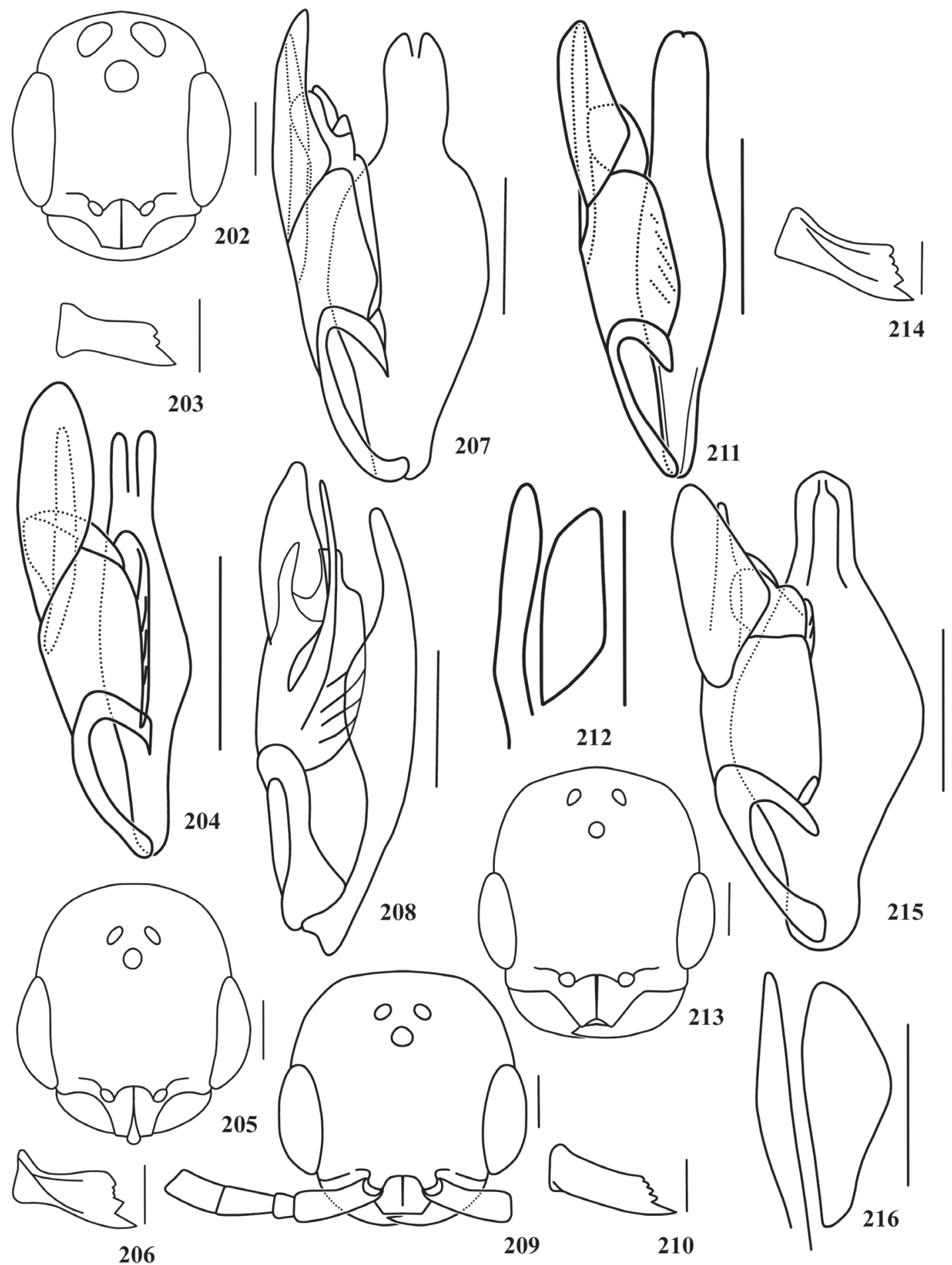

Figures 202-216. Pseudisobrachium illippum holotype male: (202) head, dorsal; (203) mandible, frontal; (204) genitalia, ventral; $P$. inchoatum holotype male: (205) head, dorsal; (206) mandible, frontal; (207) genitalia, ventral; (208) genitalia, lateral; P. intentum holotype male: (209) head, dorsal; (210) mandible, frontal; (211) genitalia, ventral; (212) paramere, full; P. jamaicense holotype male: (213) head, dorsal; (214) mandible, frontal; (215) genitalia, ventral; (216) paramere, full. Bar $=250 \mu \mathrm{m}$. 


\section{Pseudisobrachium krombeini Evans, 1961}

Figs 217-219

Holotype, male. [U.S.A.], N[ew] Mex[ico], Albuquerque, 14-15.VIII.1959, at light, K. V. Krombein col. (USNM, \#65161).

Descriptions. Male. Length $4.0 \mathrm{~mm}$. Dark castaneous. Mandible with four apical teeth, uppermost tooth wide and blunt, upper three teeth smaller than sublower. Clypeus with broad trapezoidal median lobe, apical margin concave, median carina bifurcated apically. Antenna thick, segment XI 1.15 as long as wide, pubescence of basal flagellomeres short and appressed, with some suberect setae outstanding pubescence. Eye long-haired. Frons strongly coriaceous, punctures shallow. WH 0.91x LH; WF $0.72 x$ WH, WF 1.49x HE, DAO 0.17x WF, OOL 1.0x WOT, posterior ocellus distant from vertex crest $1.2 x$ DAO. Vertex badly convex, corner rounded. VOL 1.1x HE. Temple parallel anteriorly. Notaulus absent. Parapsidal furrow complete. Propodeal disc $0.92 \mathrm{x}$ as wide as long, $0.72 \mathrm{x}$ as high as long, median carina conspicuous, incomplete, basal triangle areolate, posterior carina absent. Mesopleuron with coriaceous callus. Fore wing with discoidal vein nearly unpigmented basally, interstitial with median vein. Genitalia: paramere much wide basally, with ventral arm 2.0x wider than dorsal; aedeagus mesoconvex, apex wider than base, not reaching apex of dorsal arm of paramere.

\section{Pseudisobrachium laticeps Kieffer, 1904}

Figs 220-221

Holotype, female. BOLIVIA, Rio Beni, Salinas, 1891, Balzan col. (MCSN).

Descriptions. Female. Length $4.39 \mathrm{~mm}$. Head and mesosoma dark castaneous, metasoma castaneous. Mandible with three of equal size apical teeth. Clypeus with very wide trapezoidal median lobe, apical margin straight, triangular medially in frontal view, median carina high in profile. Eye light, subcircular, with one facet. Malar space as long as eye length. Frons polished, densely punctuated with small punctures, with unpunctuated longitudinal median band. Anterior half of head with punctures in longitudinal channeled lines in dorsal, lateral and ventral sides. Head with sides barely convex and parallel, vertex straight, corner broadly rounded, $1.05 \mathrm{x}$ as long as wide, $1.5 \mathrm{x}$ as wide as maximum width of mesosoma. Pronotal disc, mesoscutum and propodeal disc in ratio of 21:15:15. Mid tibia strongly spinose. Metasoma with short petiole.

Remarks. The head is conspicuously wide, much wider when compared with the head of the females of other species. The punctures in channeled lines are peculiar to this species and $P$. distinguendum.

\section{Pseudisobrachium Iatum Waichert \& Azevedo, 2004 Figs 222-226}

Pseudisobrachium acuminatum Waichert \& Azevedo, 2004. Syn. nov. Holotype, male. BRAZIL, E[spírito] S[anto], S[anta] Teresa, Est[ação] Biol[ógica de] Santa Lúcia, 23.VI.2001, sweeping, C.O. Azevedo \& R. Kawada col. (UFES).
Descriptions. Male. Length $2.8 \mathrm{~mm}$. Head black, mesosoma dark castaneous, prothorax lighter; fore wing subhyaline. Mandible with five apical teeth, upper four small and nearly of equal size. Clypeus with trapezoidal median lobe, apical margin angularly concave, median carina bifurcated apically. Antennal segment XI 3.5x as long as wide; pubescence of basal flagellomeres subappressed, with some erect setae outstanding pubescence. Frons coriaceous, punctures shallow. WH 0.95x LH, WF 0.57x WH, WF 1.08x HE; DAO 0.2x WF; OOL $1.4 \mathrm{x}$ WOT, posterior ocellus distant from vertex crest $0.75 \mathrm{x}$ DAO. Vertex convex, occipital carina partially visible in dorsal view. VOL $0.98 x$ HE. Temple divergent anterad. Notaulus present on anterior fourth of mesoscutum. Propodeal disc $0.98 \mathrm{x}$ as wide as long, $0.68 \mathrm{x}$ as high as long, median and posterior carinae absent, basal triangle small, otherwise propodeum areolate. Mesopleuron with weakly coriaceous callus. Hypopygium with posterior margin with rounded emargination. Fore wing with discoidal vein weakly pigmented basally, interstitial with median vein. Genitalia: paramere with ventral arm $2.5 x$ wider than dorsal arm, ventral arm wider medially, dorsal arm evenly narrow and arched; aedeagus nearly evenly narrow, its apex not reaching apex of dorsal arm of paramere.

Remarks. The general feature of this species is similar to that of $P$. formosum, but the latter has the occipital carina strongly developed and the temples more convergent posterad.

\section{Pseudisobrachium acuminatum Waichert \& Azevedo, 2004, Syn. nov.}

Figs 227-231

Holotype, male. BRAZIL, E[spírito] S[anto], S[anta] Teresa, Est[ação] Biol[ógica de] Santa Lúcia, 23.VIII.2001, sweeping, C.O. Azevedo \& R. Kawada col. (UFES).

Descriptions. Male. Length $2.7 \mathrm{~mm}$. Head black, mesosoma dark castaneous with prothorax distinctly lighter; fore wing subhyaline. Mandible with five apical teeth, upper four small and nearly of equal size. Clypeus with trapezoidal median lobe, apical margin angularly concave, median carina straight and angled in profile. Antennal segment XI 3.3x as long as wide; pubescence of basal flagellomeres subappressed, with some erect setae outstanding pubescence. Frons coriaceous, punctures shallow and inconspicuous. WH 0.91x LH, WF 0.58x WH, WF 1.17x HE; DAO 0.19x WF; OOL 1.54x WOT, posterior ocellus distant from vertex crest $0.75 \mathrm{x}$ DAO. Vertex slightly convex. VOL $0.94 \mathrm{x}$ HE. Temple divergent anterad. Occipital carina visible in dorsal view. Notaulus very short, anterior. Propodeal disc $0.9 \mathrm{x}$ as wide as long, $0.73 \mathrm{x}$ as high as long, entirely areolate, with longitudinal depression instead of median carina, posterior carina absent. Mesopleuron with coriaceous callus. Fore wing with discoidal vein weakly pigmented, interstitial with median vein. Hypopygium with posterior margin with rounded emargination. Genitalia: paramere with ventral arm more than $2.5 \mathrm{x}$ wider than dorsal arm; aedeagus mesoconvex, little dilated, its apex not reaching apex of dorsal arm of paramere. 
Remarks. The illustration of hypopygium is of one paratype with same data of holotype except for the date 31.V.2001 (UFES). The general features of the body, especially the head, the clypeus, the posterior margin of the hypopygium, the dorsal and the ventral arms of the paramere and the shape of the aedeagus of this species are very similar to those of $P$. latum and I propose it as a new junior synonym for $P$. latum.

\section{Pseudisobrachium Iuisae Vargas \& Terayama, 2002 Figs 232-233}

Holotype, female. COLOMBIA, Choco, Rio Sucio, Atrato Truando, 1.XI.[19]94, Luisa Mendoza col., JVIC 0128 (IAVH-E 86693).

Descriptions. Female. Length $3.5 \mathrm{~mm}$. Dark castaneous, nearly black. Mandible with three conspicuous apical teeth. Clypeus with broad trapezoidal median lobe, median carina high, angled and bifurcated before apical margin, apical margin with some bristles. Eye dark castaneous, elliptical, with one facet. Malar space much longer than eye length. Frons polished, punctures dense, shallow, large and subcontiguous. Head with sides straight and subparallel, vertex convex, $1.15 \mathrm{x}$ as long as wide. Pronotal disc, mesoscutum and propodeal disc in ratio of 18:18:18. Mid tibia strongly spinose. Metasoma not petiolate.

\section{Pseudisobrachium magnum Waichert \& Azevedo, 2004} Figs 234-237

Holotype, male. BRAZIL, E[spírito] S[anto], S[anta] Teresa, Est[ação] Biol[ógica de] Santa Lúcia, 30.I.2001, sweeping, C.O. Azevedo \& R. Kawada col. (UFES).

Descriptions. Male. Length $5.9 \mathrm{~mm}$. Black; fore wing subhyaline. Mandible with five apical teeth, upper three smaller than sublower. Clypeus with trapezoidal median lobe, apical margin convex medially, median carina becoming polished area apically. Antennal segment XI 2.3x as long as wide; pubescence of basal flagellomeres subappressed, with some erect setae outstanding pubescence. Frons strongly coriaceous, punctures shallow and large. WH 0.97x LH, WF 0.64x WH, WF 1.31x HE; DAO $0.14 \mathrm{x}$ WF; OOL 1.47x WOT, posterior ocellus distant from vertex crest 1.93x DAO. Vertex convex. VOL 0.81x HE. Temple divergent anterad. Pronotal disc with transverse elevation medially. Notaulus present on anterior third of mesoscutum. Propodeal disc $0.98 \mathrm{x}$ as wide as long, $0.66 \mathrm{x}$ as high as long, median carina incomplete, posterior carina indistinguishable from strigulation, basal triangle small, otherwise propodeum mostly strigulate. Mesopleuron with polished callus. Fore wing with discoidal vein weakly pigmented, nearly interstitial with median vein. Genitalia: paramere with ventral arm 3.0x wider than dorsal arm; aedeagus mesoconvex, with subapical constriction, its apex about aligned with apex of dorsal arm of paramere.

\section{Pseudisobrachium manni Evans, 1961}

Figs 238-239

Holotype, female. GUAT[EMALA], Mexico, V.[19]24, W. M. Mann col. (USNM, \#65158).
Descriptions. Female. Length $4.28 \mathrm{~mm}$. Black. Mandible with four apical teeth, uppermost one small. Clypeus with very broad trapezoidal median lobe, apical margin straight with few bristles, median carina not high. Eye circular, with one facet. Malar space about as long as eye length. Frons polished, densely punctuated. Head with sides straight and nearly parallel, vertex nearly straight, $1.22 \mathrm{x}$ as long as wide. Pronotal disc, mesoscutum and propodeal disc in ratio of 16:17:16. Mid tibia strongly spinose. Metasoma with short petiole.

\section{Pseudisobrachium masoni Evans, 1967 Figs $240-243$}

Holotype, male. MEXICO, Durango, 3 mi. Ethiopian El Salto, 8500 feet, 10.VII.1964, W. R. M. Mason col. (CNCI, \#18354).

Descriptions. Male. Length $6.0 \mathrm{~mm}$. Black. Mandible with five apical teeth, progressively larger ventrad. Clypeus with subtrapezoidal median lobe, median carina duplicated basally and bifurcated apically. Antennal segment XI about 2.0 as long as wide, pubescence of basal flagellomeres subappressed, with some long suberect setae outstanding pubescence. Frons polished, punctures small. WH 0.85x LH, WF 0.66x WH, WF $1.7 \mathrm{x}$ HE, DAO $0.13 \mathrm{x}$ WF, OOL $1.5 \mathrm{x}$ WOT, posterior ocellus distant from vertex crest $2.55 \mathrm{x}$ DAO. Vertex rounded. VOL 1.29x HE. Notaulus well impressed, incomplete posteriorly. Propodeal disc reticulate, $0.94 \mathrm{x}$ as wide as long, $0.71 \mathrm{x}$ as high as long, median carina complete, posterior carina absent. Mesopleuron with polished callus. Fore wing with discoidal vein weakly pigmented, not interstitial with median vein. Genitalia: paramere with ventral arm 1.4X wider than dorsal arm; aedeagus mesoconvex, its apex much not reaching apex of dorsal arm of paramere.

\section{Pseudisobrachium merklei Bruch, 1917} Figs 244-245

Syntype female: ARGENTINA, La Plata, b. Solenopsis saevissima Sm., 1917, C. Bruch col. (NHME).

Descriptions. Female. Length $4.0 \mathrm{~mm}$. Dark castaneous. Mandible with four apical teeth, upper two very small. Clypeus with broad trapezoidal median lobe, margin with some bristles, median carina high. Eye light, elliptical, with one facet. Malar space about as long as eye length. Frons polished, densely punctuated, except for median longitudinal band. Head with sides subparallel, vertex slightly convex, $1.24 \mathrm{x}$ as long as wide. Length of pronotal disc, mesoscutum and propodeal disc in ratio of 24:18:20. Propodeal disc strongly constricted anteriorly. Mid tibia strongly spinose. Metasoma not petiolate.

Remarks. I found three specimens at Wasmann Collection whose data present on the label correspond to those indicated in the original description. Allthough they are not indicated as syntype series I was going to treat them as syntypes and select one to be the lectotype. However while revising the final editor version of this manuscript, Marcelo T. Tavares, who was visiting Museo de la Plata and informed me that there were four specimens with label of "typus \#2063" of P. merklei. So I 


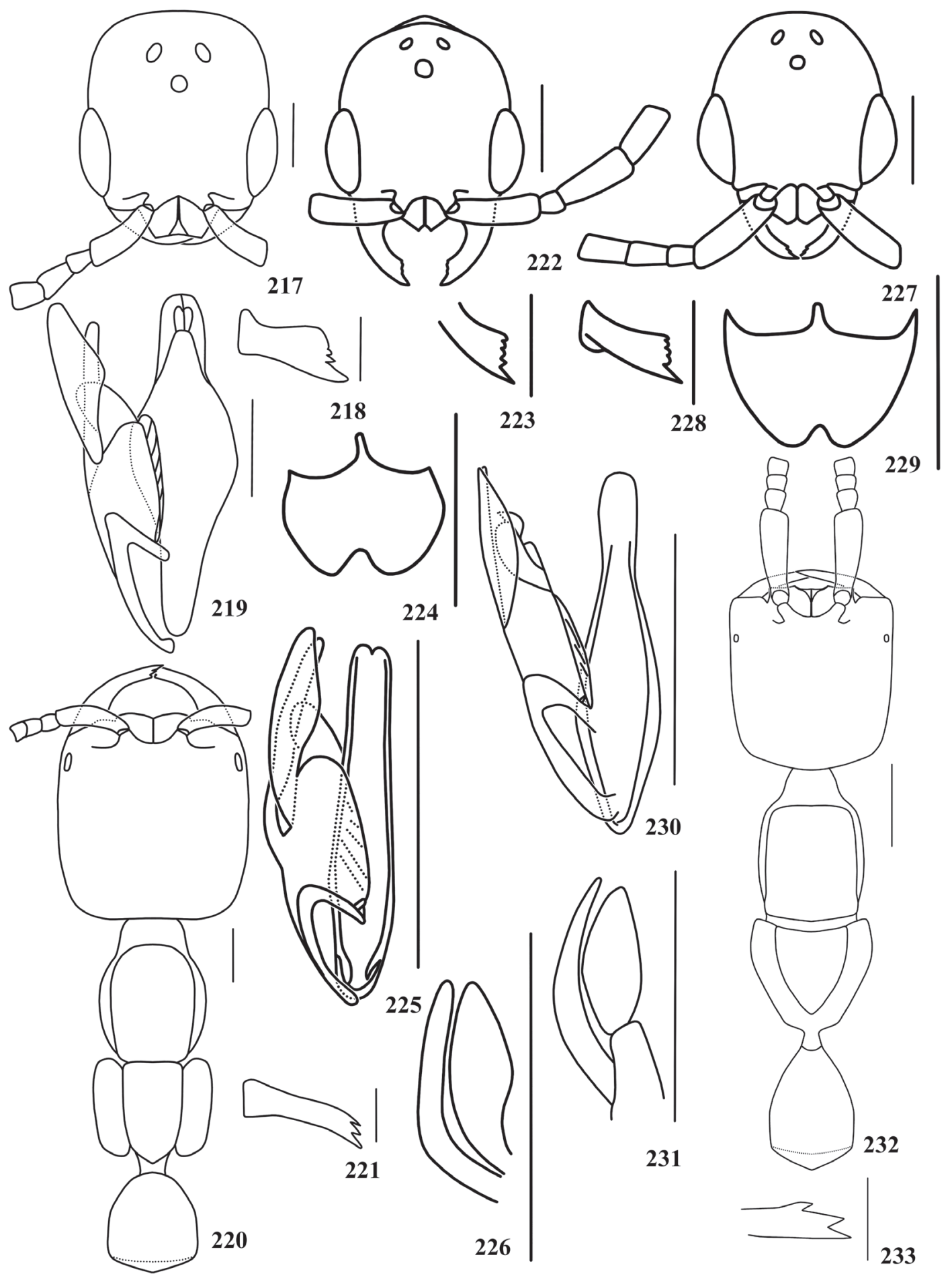

Figures 217-233. Pseudisobrachium krombeini holotype male: (217) head, dorsal; (218) mandible, frontal; (219) genitalia, ventral; $P$. laticeps holotype female: (220) head and mesosoma, dorsal; (221) mandible, frontal; P. latum holotype male: (222) head, dorsal; (223) mandible, frontal; (224) hypopygium, ventral; (225) genitalia, ventral; (226) paramere, full; P. acuminatum holotype male: (227) head, dorsal; (228) mandible, frontal; (229) hypopygium of paratype, ventral; (230) genitalia, ventral; (231) paramere, full; P. luisae holotype female: (232) head and mesosoma, dorsal; (233) mandible, frontal. Bar $=250 \mu \mathrm{m}$. 

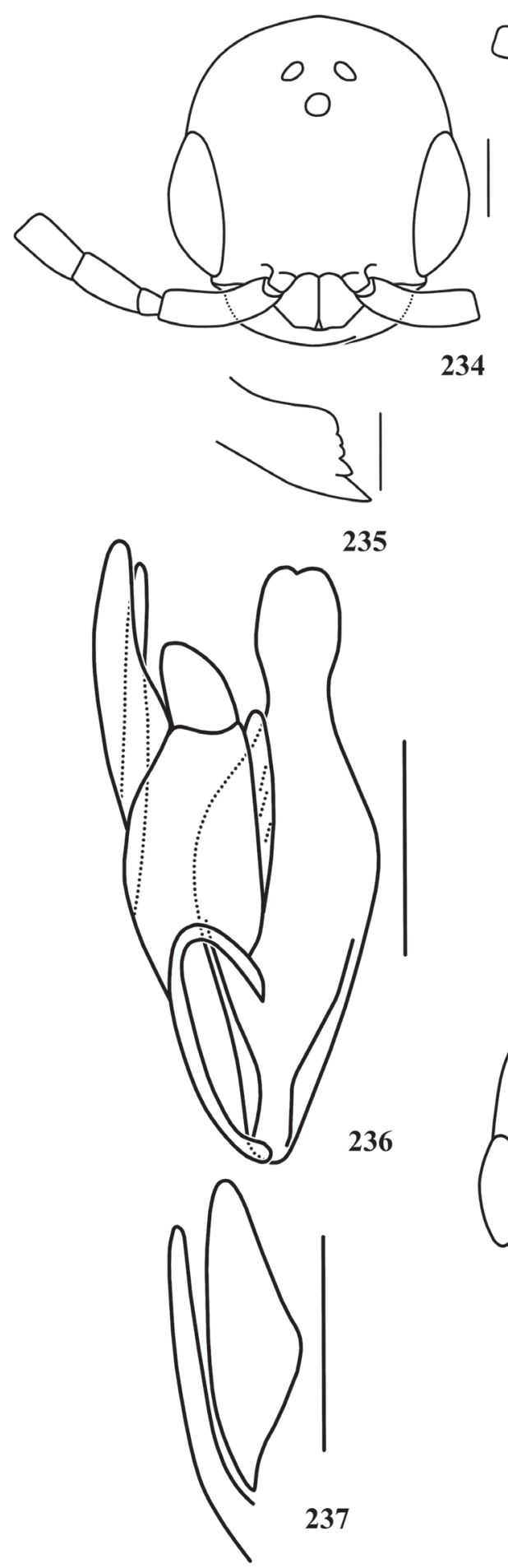
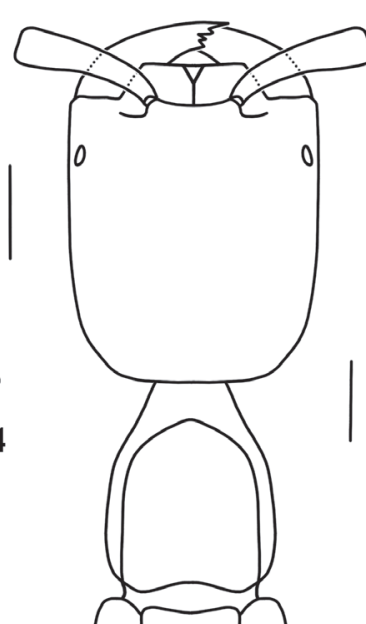

235

236
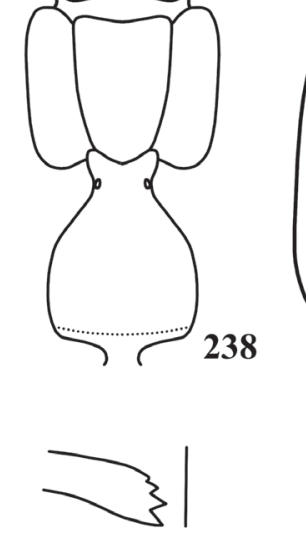

239
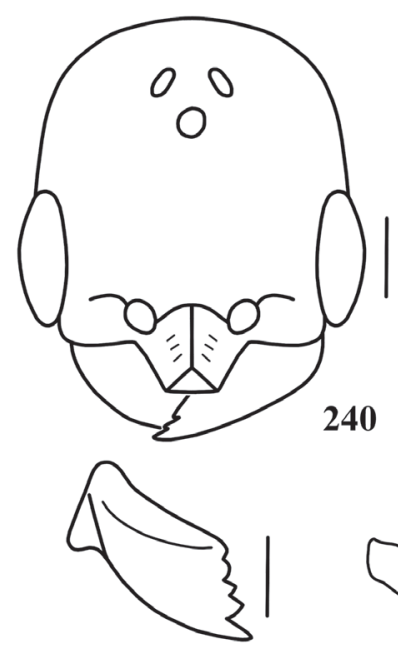

241

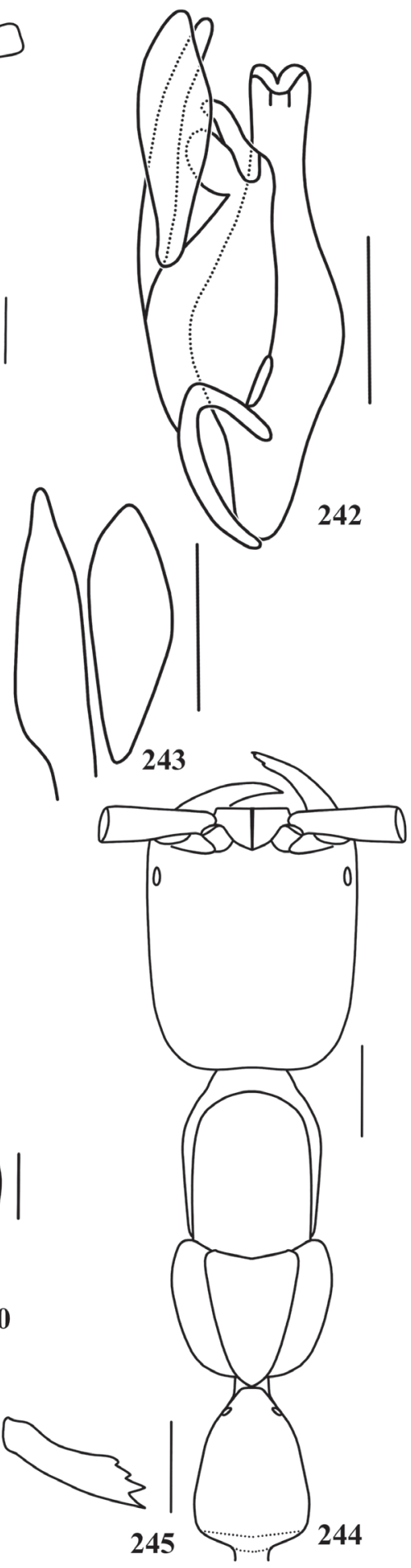

Figures 234-245. Pseudisobrachium magnum holotype male: (234) head, dorsal; (235) mandible, frontal; (236) genitalia, ventral; (237) paramere, full; P. manni holotype female: (238) head and mesosoma, dorsal; (239) mandible, frontal; P. masoni holotype male: (240) head, dorsal; (241) mandible, frontal; (242) genitalia, ventral; (243) paramere, full; P. merklei syntype female: (244) head and mesosoma, dorsal; (245) mandible, frontal. $\mathrm{Bar}=250 \mu \mathrm{m}$. 
decided not to designate a lectotype following the International Code of Zoological Nomenclature, which suggests to examine all syntype series before the lectotype designation.

\section{Pseudisobrachium micheneri Evans, 1961 Figs $246-249$}

Holotype, male. MEX[ICO], Puebla, 5 mi NE of Teziutlan, 4700 f[ee]t, 27.VI.[19]53, Univ[ersity] Kans[as] Mex[ican] Expedition (SEMC).

Descriptions. Male. Length $6.6 \mathrm{~mm}$. Black; fore wing subhyaline. Mandible with five large apical teeth, of equal size. Clypeus with short trapezoidal median lobe. Antenna with segment XI about 2.6x as long as wide, pubescence appressed and longer than half diameter of basal flagellomeres, with some erect setae outstanding pubescence. Eye bulging, long-haired. Frons polished, with dense punctures. WH 1.04x LH; WF 0.59x WH, WF 1.3x HE, DAO 0.15x WF, OOL 1.25x WOT, frontal angle of ocellar triangle acute, posterior ocellus distant from vertex crest 2.0x DAO, anterior margin of anterior ocellus closed to eye top imaginary line. Vertex straight, corner broadly rounded. VOL $0.87 \mathrm{x}$ HE. Pronotal disc rugulose. Mesoscutum densely punctuated. Notaulus complete. Scutellar groove wide and deep. Propodeum rugulose-rugulose, disc $0.87 \mathrm{x}$ as wide as long, $0.67 \mathrm{x}$ as high as long, median carina complete, posterior carina indistinguishable. Mesopleuron with polished callus contrasting from rugulose area. Fore wing with discoidal vein weakly pigmented basally, not interstitial with median vein. Genitalia: paramere with ventral arm $2.8 \mathrm{x}$ wider than dorsal; aedeagus mesoconvex, apex wide, its apex not reaching apex of dorsal arm of paramere, base very narrow.

\section{Pseudisobrachium michoacanum Evans, 1961 Figs 250-253}

Holotype, male. MEX[ICO], Micho[acan], Tuxpan, 6000', 6.VII.[19]59, H. E. Evans col. (MCZH, \#30275).

Descriptions. Male. Length $4.1 \mathrm{~mm}$. Dark castaneous; fore wing subhyaline. Mandible with five apical teeth, upper three smaller than sublower. Clypeus with trapezoidal median lobe, median carina bifurcated apically. Antenna with segment XI $1.43 \mathrm{x}$ as long as wide, pubescence of basal flagellomeres appressed, with some long erect setae outstanding pubescence. Eye long-haired. Frons coriaceous, punctures shallow. WH 0.96x LH; WF 0.65x WH, WF 1.48x HE, DAO 0.15x WF, OOL 1.43x WOT, posterior ocellus distant from vertex crest $1.7 \mathrm{x}$ DAO. Vertex slightly convex, corner rounded. VOL $0.97 x$ HE. Notaulus present on anterior third of mesoscutum. Propodeal disc $0.97 \mathrm{x}$ as wide as long, $0.65 \mathrm{x}$ as high as long, median carina incomplete, basal triangle large and strongly rugulose, posterior carina absent; declivity areolate-foveolate; lateral of propodeum coriaceous-aciculate. Mesopleuron with coriaceous callus. Fore wing with discoidal vein unpigmented, not interstitial with median vein. Genitalia: paramere with ventral arm 2.6x wider than dorsal; aedeagus mesoconvex, its apex slightly not reaching apex of dorsal arm of paramere.

\section{Pseudisobrachium minimum Evans, 1961 \\ Figs 254-256}

Holotype, male. [U. S. A.], N[ew] Mex[ico], city of Rocks S[tate] P[ar]k, 17.VII.1959, at light, K. V. Krombein col. (USNM, \#65160).

Descriptions. Male. Length $3.33 \mathrm{~mm}$. Black. Mandible with four nearly of equal size apical teeth. Clypeus with trapezoidal median lobe, apical margin barely concave, median carina bifurcated apically. Antenna thick, segment XI as long as wide, pubescence of basal flagellomeres appressed, with some long suberect setae outstanding pubescence. Frons weakly coriaceous, punctures shallow. WH 0.95x LH, WF 0.63x WH, WF 1.29x HE, DAO 0.15x WF, OOL 1.19x WOT, posterior ocellus distant from vertex crest $0.83 x$ DAO. Vertex broadly convex. VOL $1.22 x$ HE. Notaulus absent. Propodeal disc $0.88 x$ as wide as long, $0.71 \mathrm{x}$ as high as long, median carina incomplete, weakly rugulose, posterior carina absent. Mesopleuron with polished callus. Fore wing without discoidal vein. Genitalia: paramere with ventral arm $1.5 \mathrm{x}$ wider than dorsal; aedeagus mesoconvex, apex rounded with little emargination, not reaching apex of dorsal arm of paramere.

\section{Pseudisobrachium minutissimum Evans, 1961 Figs 257-259}

Holotype, male. MEXICO, Baja California, Punta Lobos, 1 mile SE. of Todos Santos, 25.XII.1958, H. B. Leech col. (CASC, \#6870).

Descriptions. Male. Length $2.3 \mathrm{~mm}$. Castaneous. Mandible with four nearly of equal size apical teeth. Clypeus with trapezoidal median lobe, median carina bifurcated apically. Antennal segment XI barely longer than wide, pubescence subappressed, longer than half of diameter of basal flagellomeres, with some long erect setae outstanding pubescence. Eye small. Frons coriaceous, punctures inconspicuous. WH 0.92x LH, WF $0.67 \mathrm{x}$ WH, WF 1.5x HE, DAO 0.13x WF, OOL 1.5x WOT, posterior ocellus distant from vertex crest 1.2x DAO. Vertex convex. VOL 1.3x HE. Pronotal disc depressed anteriorly. Notaulus inconspicuous, present on anterior third of mesoscutum. Propodeal disc $0.73 \mathrm{x}$ as wide as long, $0.63 \mathrm{x}$ as high as long, median carina incomplete, posterior carina absent. Mesopleuron with callus indistinguishable. Fore wing with discoidal vein unpigmented, interstitial with median vein. Genitalia: paramere with ventral arm about $1.5 \mathrm{x}$ wider than dorsal arm; aedeagus mesoconvex, its apex not reaching apex of dorsal arm of paramere.

\section{Pseudisobrachium montivagum Evans, 1964 Figs $260-262$}

Holotype, male. Omilteme, Guerrero, Aug[ust], H. H. S[mith] col., Godman-Salvin Coll[ection], 1904-1 (BMNH, \#13368). Genitalia slide \#HE499.

Descriptions. Male. Length $4.71 \mathrm{~mm}$. Black. Mandible with five apical teeth, upper four small and nearly of equal size. Clypeus with quadrate median lobe, apical margin con- 

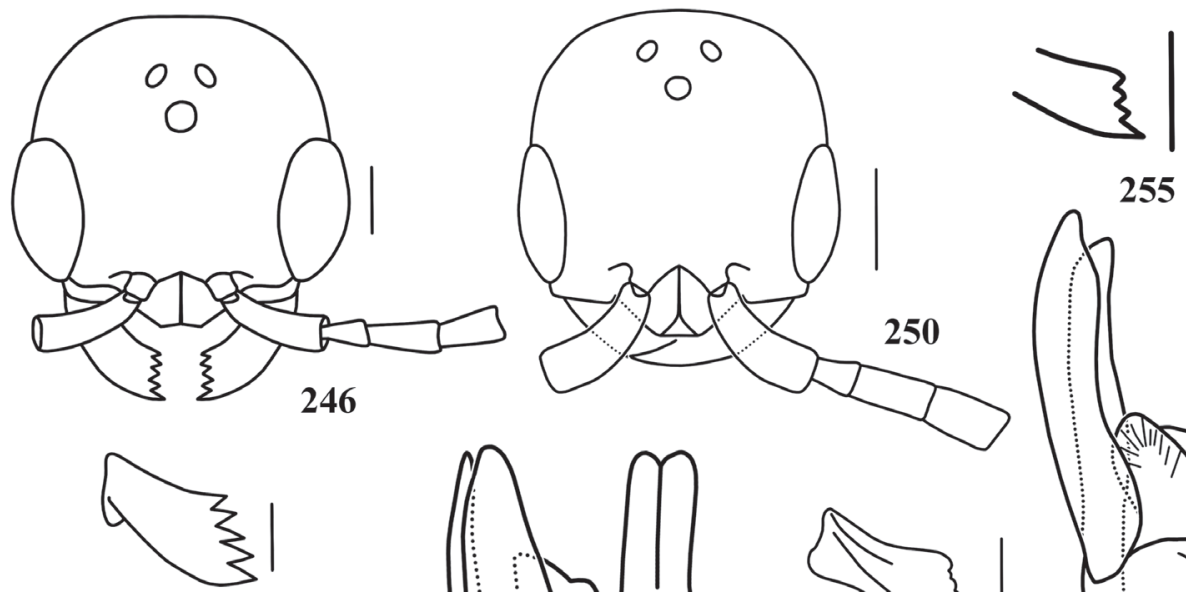

247
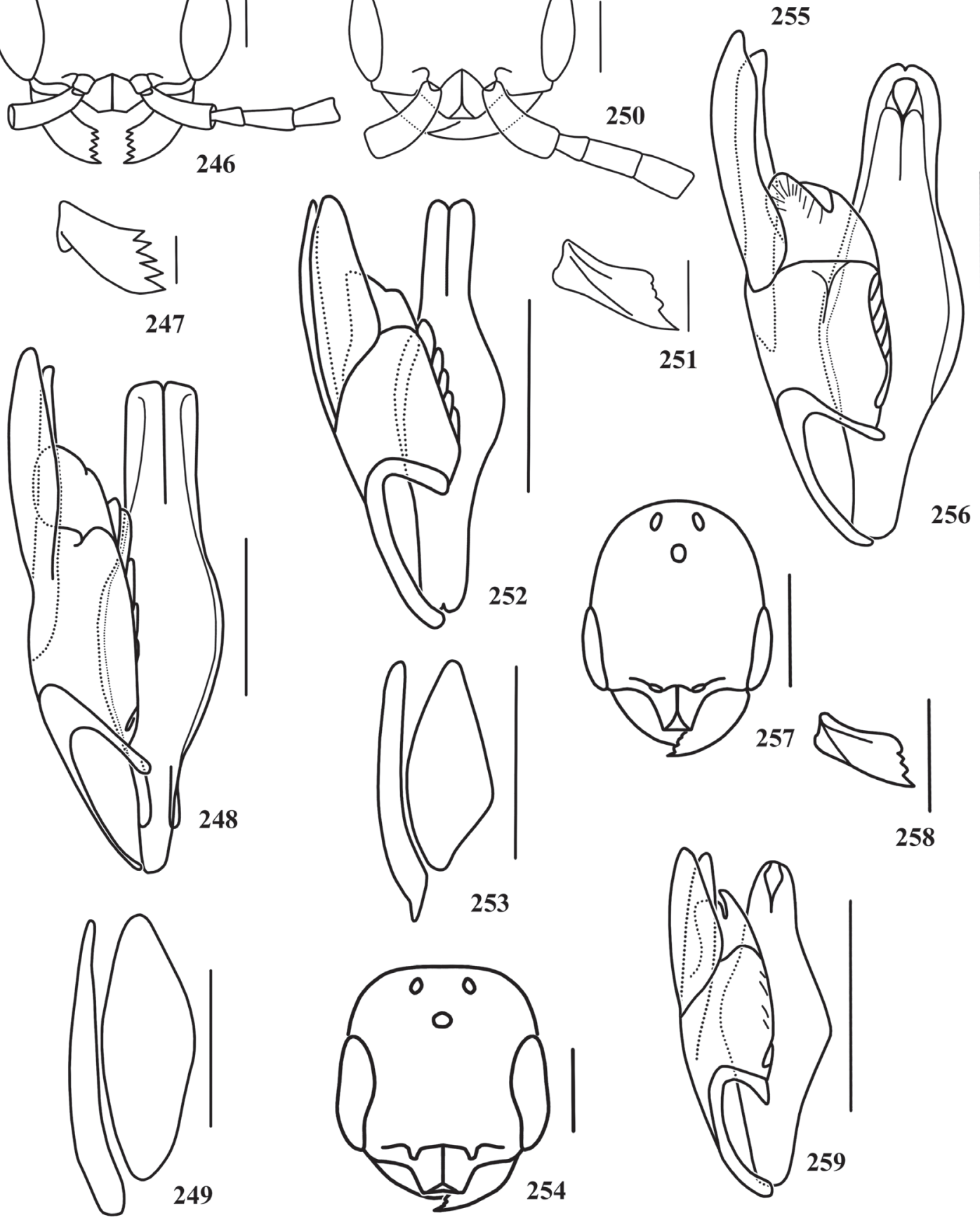

Figures 246-259. Pseudisobrachium micheneri holotype male: (246) head, dorsal; (247) mandible, frontal; (248) genitalia, ventral; (249) paramere, full; P. michoacanum holotype male: (250) head, dorsal; (251) mandible, frontal; (252) genitalia, ventral; (253) paramere, full; P. minimum holotype male: (254) head, dorsal; (255) mandible, frontal; (256) genitalia, ventral; P. minutissimum holotype male: (257) head, dorsal; (258) mandible, frontal; (259) genitalia, ventral. Bar $=250 \mu \mathrm{m}$. 
vex and broader than base, median carina slightly high in profile. Antenna segments wider distally, segment XI 2.6x as long as wide, pubescence of basal flagellomeres subappressed with few setae suberect outstanding pubescence. Eye small. Frons polished, punctures small. WH 0.94x LH, WF 0.55x WH, WF 1.2x HE, DAO 0.16x WF, OOL 1.76x WOT, posterior ocellus distant from vertex crest $1.38 \mathrm{x}$ DAO. Vertex broadly convex. VOL 1.23x HE. Notaulus well impressed, incomplete both anterior and posteriorly. Propodeal disc $0.74 \mathrm{x}$ as wide as long, $0.56 \mathrm{x}$ as high as long, median carina incomplete, posterior carina indistinguishable from rugulation. Mesopleuron with polished callus. Fore wing with discoidal vein little pigmented basally, not interstitial with median vein. Genitalia: paramere with ventral arm 2.6x wider than dorsal; aedeagus mesoconvex, its apex slightly not reaching apex of dorsal arm of paramere.

\section{Pseudisobrachium mrazi Ogloblin, 1925}

Fig. 263

Holotype, female. BRAZIL, São Paulo, J. Mráz col. (NMPC, not found).

Descriptions. Female. Length $5.7 \mathrm{~mm}$. Black. Mandible with four apical teeth. Clypeus with angulate median lobe. Eye elliptical. Frons shining, punctures dense and large. Head with sides slightly convex, slightly diverging anteriorly, so that head is wider at eye level, vertex slightly concave, $1.14 \mathrm{x}$ as long as wide. Pronotal disc, mesoscutum and propodeal disc in ratio of 26:24:22. Mid tibia strongly spinose. Metasoma with short petiole.

Remarks. The descriptions and the illustration are extracted on the original publication. Ogloblin (1925) described the eyes as elliptical, but in the illustration the eyes are circular.

\section{Pseudisobrachium nigriculum Evans, 1961 Figs 264-266}

Holotype, male. MEX[ICO], H[i]d[al]go, Zimapán, 1114.VI.[19]51, at light, H. E. Evans col. (MCZH, \#30273).

Descriptions. Male. Length $3.3 \mathrm{~mm}$. Dark castaneous; fore wing hyaline. Mandible with five apical teeth, upper three teeth smaller than sublower. Clypeus with trapezoidal median lobe, median carina bifurcated apically. Antenna with segment XI $1.55 \mathrm{x}$ as long as wide, pubescence of basal flagellomeres subappressed and long, with some long erect setae outstanding pubescence. Eye somewhat bulging, long-haired. Frons coriaceous, punctures shallow. WH 0.99x LH; WF 0.57x WH, WF 1.0x HE, ocelli large, DAO 0.29x WF, OOL 0.56x WOT, posterior ocellus close to vertex crest, anterior margin of anterior ocellus reaching eye top imaginary line. Vertex slightly convex, corner rounded. VOL 0.62x HE. Notaulus present on anterior half of mesoscutum. Propodeal disc $0.76 \mathrm{x}$ as wide as long, $0.51 \mathrm{x}$ as high as long, median carina complete, basal triangle weakly rugulose, disc otherwise polished, posterior carina absent; declivity and lateral of propodeum aciculate. Mesopleuron with polished callus. Fore wing with discoidal vein unpigmented, not interstitial with median vein. Genita- lia: paramere with ventral arm $1.8 \mathrm{x}$ wider than dorsal; aedeagus mesoconvex, apex rounded.

\section{Pseudisobrachium ogloblini Ogloblin, 1925}

Fig. 267

Holotype female: BRAZIL, São Paulo, J. Mráz col. (NMPC, not found).

Descriptions. Female. Length $2.8 \mathrm{~mm}$ long; black; mandible with four apical teeth, subupper smaller; eye light and elliptical; frons strongly coriaceous, punctures dense; head with sides straight and parallel, $1.3 \mathrm{x}$ as long as wide; vertex slightly concave; pronotal disc, mesoscutum and propodeal disc in ratio of 8:7:8; mid tibia spinose and metasoma with short petiole.

Remarks. I was not able to find the female holotype from Brazil, São Paulo, collected by J. Mráz col. (NMPC). Diagnosis and illustration extracted on the original publication.

\section{Pseudisobrachium obscurum Evans, 1961 Figs $268-273$}

Holotype, male. [U.S.A.], Tex[as], Culberson Co., Pine Springs, 13-16.VII.19[56], [at light], E. G. Matthews col. (MCZH, \#30278).

Descriptions. Male. Length $4.7 \mathrm{~mm}$. Dark castaneous; fore wing hyaline. Mandible with five apical teeth, upper four small and nearly of equal size. Clypeus with trapezoidal median lobe. Antenna thick, segment XI 1.73x as long as wide, pubescence of basal flagellomeres appressed and short, with some long erect setae outstanding pubescence. Eye long-haired. Frons coriaceous, punctures shallow. WH 0.94x LH; WF 0.61x WH, WF 1.09x HE, ocelli large, DAO 0.25x WF, OOL 0.6x WOT, posterior ocellus distant from vertex crest $0.78 \mathrm{x}$ DAO. Vertex convex, corner rounded. VOL $0.7 x$ HE. Notaulus present on anterior third of mesoscutum. Propodeal disc $0.95 \mathrm{x}$ as wide as long, $0.66 \mathrm{x}$ as high as long, median carina incomplete, basal triangle rugulose, disc otherwise aciculate, posterior carina absent; declivity aciculate; lateral of propodeum aciculate. Mesopleuron with coriaceous low callus. Fore wing with discoidal vein unpigmented, not interstitial with median vein. Genitalia: paramere with ventral arm $2.6 \mathrm{x}$ wider than dorsal; aedeagus mesoconvex, apex with margin converging apicad, nearly aligned with apex of dorsal arm of paramere, base narrow.

Allotype ? female: [U.S.A.], Ariz[ona], Pinal Co., Sacaton, 1935, from soil in cotton field, C46-BC3, L. D. Christenson col. (USNM).

Descriptions. Female. Length $2.2 \mathrm{~mm}$. Castaneous. Mandible with four apical teeth in oblique series. Clypeus with subtrapezoidal median lobe, median carina bifurcated apically. Eye pale, subcircular, with one facet. Malar space longer than as eye length. Frons polished, punctures shallow, with narrow unpunctuated median longitudinal band. Head with sides straight and slightly divergent anteriorly, vertex nearly straight, $1.29 \mathrm{x}$ as long as wide. Pronotal disc, mesoscutum and propodeal disc in ratio of 21:19:16, with suture separating pronotal disc and mesoscutum. Mid tibia spinose. Metasoma with short petiole. 


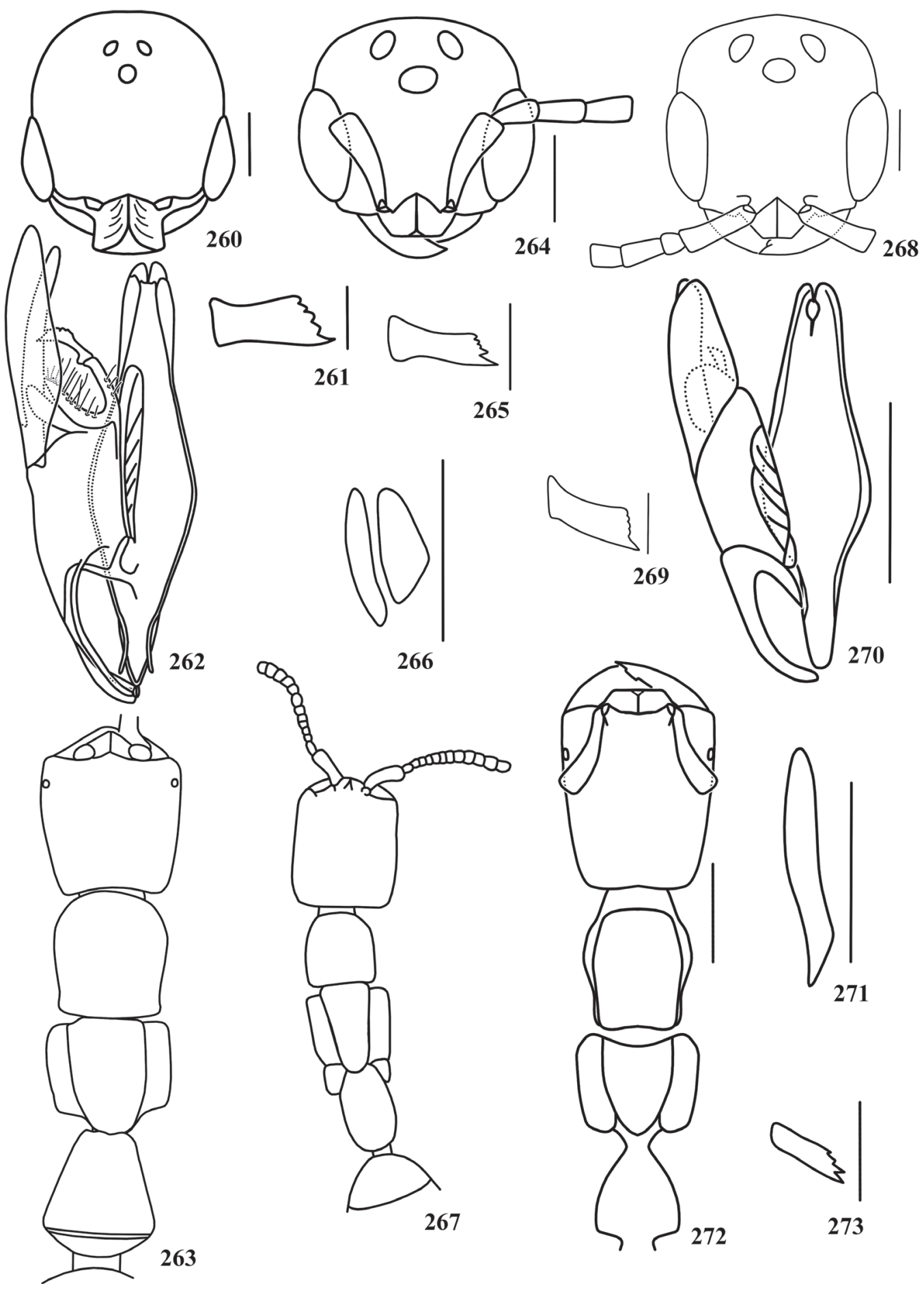

Figures 260-273. Pseudisobrachium montivagum holotype male: (260) head, dorsal; (261) mandible, frontal; (262) genitalia, ventral; $P$. mrazi original illustration, female: (263) head and mesosoma, dorsal; P. nigriculum holotype male: (264) head, dorsal; (265) mandible, frontal; (266) paramere, full; P. ogloblini original illustration: (267) head and mesosoma; P. obscurum holotype male: (268) head, dorsal; (269) mandible, frontal; (270) genitalia, ventral; (271) dorsal arm of paramere, full; allotype female: (272) head and mesosoma, dorsal; (273) mandible, frontal. Bar $=250 \mu \mathrm{m}$. 


\section{Pseudisobrachium opimum Waichert \& Azevedo, 2004 Figs 274-277}

Holotype, male. BRAZIL, E[spírito] S[anto], S[anta] Teresa, Est[ação] Biol[ógica de] Santa Lúcia, 31.V.2001, sweeping, C.O. Azevedo \& R. Kawada col. (UFES).

Descriptions. Male. Length $4.3 \mathrm{~mm}$. Dark castaneous, head darker and thorax lighter; fore wing subhyaline. Mandible with five apical teeth, upper three smaller than others. Clypeus with subtrapezoidal median lobe, apical margin straight, median carina high. Antennal segment XI 2.8x as long as wide; pubescence of basal flagellomeres subappressed, with few erect setae outstanding pubescence. Frons strongly coriaceous, punctures shallow. WH $0.94 \mathrm{x}$ LH, WF $0.65 \mathrm{x}$ WH, WF 1.41x HE; DAO 0.14x WF; OOL 1.59x WOT, posterior ocellus distant from vertex crest $1.67 \mathrm{x}$ DAO. Vertex slightly convex. VOL $0.89 x$ HE. Temple divergent anterad. Notaulus on anterior half of mesoscutum. Propodeal disc $0.88 \mathrm{x}$ as wide as long, $0.63 \mathrm{x}$ as high as long, basal triangle large, median carina incomplete, posterior carina absent; declivity irregularly strigulate, lateral of propodeum coriaceous. Mesopleuron with polished callus. Fore wing with discoidal vein weakly pigmented, not interstitial with median vein. Genitalia: paramere with ventral arm 4.0x wider of dorsal arm; aedeagus mesoconvex, its apex not reaching apex of dorsal arm of paramere.

\section{Pseudisobrachium optimum Evans, 1964}

Figs 278-282

Holotype, male. BRAZIL, Santa Catarina, Nova Teutonia, $27^{\circ} 11^{\prime}$ S 52 $23^{\prime}$ W, 7.XI.1944, Fritz Plaumann col. (AMNH).

Descriptions. Male. Length $8.8 \mathrm{~mm}$. Black; fore wing yellowish, with large brown band at radial vein level; pubescence yellowish. Mandible wide apically, with five large of equal size apical teeth. Clypeus with rounded median lobe, median carina bifurcated apically. Antenna with segment XI about $2.5 \mathrm{x}$ as long as wide, pubescence subappressed, about as long as half diameters of basal segments, with some erect setae outstanding pubescence. Eye long-haired. Frons polished, punctures small. WH 0.99x LH; WF 0.64x WH, WF 1.35x HE, DAO 0.12x WF, OOL 1.29x WOT, frontal angle of ocellar triangle about right, posterior ocellus distant from vertex crest $3.5 \mathrm{x}$ DAO, anterior margin of anterior ocellus reaching eye top imaginary line. Vertex convex, corner broadly rounded. VOL $0.88 x$ HE. Notaulus well impressed, present on two anterior thirds of mesoscutum. Propodeal disc $1.2 \mathrm{x}$ as wide as long, $0.9 \mathrm{x}$ as high as long, median carina incomplete, basal triangle undefined, disc strongly rugulose, posterior carina distinct; declivity strongly rugulose; lateral of propodeum polished, surrounded by pits and depressions. Mesopleuron with weakly coriaceous callus, episternal groove very large and foveolate. Fore wing with discoidal vein weakly pigmented, interstitial with median vein. Hypopygium with posterior margin deeply emarginated, outer surface with pair of long teeth, inner surface with verti- cal keel. Genitalia: paramere with dorsal arm unusually narrow and short, ventral arm 2.6x wider than dorsal arm; vannus absent; aedeagus elliptical with five pairs of apical lobes; dorsal pair wide with rounded apex; outer pair higher and narrower than dorsal pair; ventral pair stout, membranous with surface full of semi-ball elevation; median and inner pairs as finger-shaped expansions; inner surface of dorsal slope covered by minute denticles and hairy.

Remarks. This species has the most unusual genitalia I have ever seen in this genus, with many apical lobes in the aedeagus and the cuspis strongly bilobate. Besides, the propodeum is unusually rugulose, the episternal groove of mesopleuron is unusually large and foveolate, the hypopygium has strong bi-horned emargination. In addition, this species is the only one know without vannus.

\section{Pseudisobrachium otiosum Evans, 1961 Figs 283-284}

Holotype, male. [U. S. A.], Arizona, Superior, [Pinal Co.], 7-17.VII.[19]48, D. K. G. Lloyd col. (USNM, \#65159).

Diagnosis, male. Dark castaneous, head nearly black. Mandible with five apical teeth, upper four nearly of equal size. Clypeus with trapezoidal median lobe. Antenna thick, segment $\mathrm{XI} 1.28 \mathrm{x}$ as long as wide, pubescence of basal flagellomeres appressed, with some erect setae outstanding pubescence. Frons strongly coriaceous, punctures inconspicuous. WH 0.96x LH, WF 0.71x WH, WF 1.52x HE, DAO 0.15x WF, OOL 0.94x WOT, posterior ocellus distant from vertex crest $0.38 \mathrm{x}$ DAO. Vertex convex. VOL 1.02x HE. Pronotal disc depressed forward. Notaulus inconspicuous, present on anterior half of mesoscutum. Propodeal disc $0.87 \mathrm{x}$ as wide as long, $0.6 \mathrm{x}$ as high as long, median carina incomplete, otherwise with weakly rugulose, posterior carina absent. Mesopleuron with ill-defined callus. Fore wing without discoidal vein. Metasoma missing.

\section{Pseudisobrachium pallidipes (Cameron, 1909)}

\section{Figs $285-288$}

Holotype, male. [ARGENTINA], Mendonza (BMNH).

Diagnosis, male. Length $5.4 \mathrm{~mm}$. Dark castaneous, Fore wing subhyaline. Mandible with three apical teeth, uppermost wide. Clypeus with subtrapezoidal median lobe, median carina low and straight in profile, bifurcated apically. Antennal segment XI $1.4 \mathrm{x}$ as long as wide, pubescence of basal flagellomeres appressed with erect setae outstanding pubescence. Frons coriaceous, punctures shallow. WH 1.04x LH, WF 0.6x WH, WF 1.15x $\mathrm{HE}$, ocelli large and high, DAO 0.25x WF, OOL 0.56x WOT, posterior ocellus distant from vertex crest $0.63 \mathrm{x}$ DAO, anterior margin of anterior ocellus reaching eye top imaginary line. Vertex convex, corner rounded. VOL 0.6x HE. Notaulus absent. Parapsidal furrow well-impressed, complete. Propodeal disc $0.97 \mathrm{x}$ as wide as long, 0.6x as high as long, median carina incomplete, posterior carina absent, basal triangle rugulose-areolate, disc oth- 
erwise aciculate; declivity aciculate. Mesopleuron with small callus. Fore wing with discoidal vein unpigmented, not interstitial with median vein. Genitalia: paramere with ventral arm 2.0x wider than dorsal; aedeagus mesoconvex, its apex slightly not reaching apex of dorsal arm of paramere.

\section{Pseudisobrachium argentinicum Kieffer, 1910 . Figs 289-292} \#206).

Descriptions. Male. ARGENTINA, Mendoza, 1906 (ZMHB,

Diagnosis, male. Length $4.9 \mathrm{~mm}$. Dark castaneous, fore wing hyaline. Mandible with three apical teeth, uppermost wide. Clypeus with trapezoidal median lobe, apical margin slighlty convex, median carina incomplete apically. Antennal segment XI 1.6x as long as wide, pubescence of basal flagellomeres appressed with erect setae outstanding pubescence. Frons coriaceous, punctures large and shallow. WH 1.04x LH, WF 0.61x WH, WF 1.15x HE, DAO 0.19x WF, OOL 0.61x WOT, posterior ocellus distant from vertex crest 1.0x DAO anterior margin of anterior ocellus reaching eye top imaginary line. Vertex badly convex, corner rounded. VOL 0.56x HE. Notaulus absent on posterior half. Parapsidal furrow complete. Propodeal disc $0.86 \mathrm{x}$ as wide as long, $0.58 \mathrm{x}$ as high as long, median carina incomplete, posterior carina absent, basal triangle inconspicuous; declivity coriaceous. Fore wing with discoidal vein unpigmented, not interstitial with median vein. Genitalia: paramere with ventral arm 2.6x wider than dorsal; aedeagus mesoconvex, its apex not reaching apex of dorsal arm of paramere.

Remarks. $P$. argentinicum is very similar to $P$. pallidipes and I agree with this synonym, established by Evans (1973).

\section{Pseudisobrachium pallidum Evans, 1961} Figs 293-294

Holotype, male. [U.S.A.], Ariz[ona], Juma Co., Wellton, 9.VIII.1917, C[ornell] U[niversity] Biol[ogy] Exped[ition], lot 542, sub 53, [J. C. Bradley col.] (CUIC, \#3872).

Descriptions. Male. Length $1.8 \mathrm{~mm}$, disregarding metasoma. Castaneous; fore wing weakly subhyaline. Mandible with five apical teeth, upper three smaller than sublower. Clypeus with trapezoidal median lobe, median carina bifurcating since base to apical corner. Antenna with segment XI about as long as wide, pubescence of basal flagellomeres appressed and short, with some erect setae outstanding pubescence. Eye large, long-haired. Frons coriaceous, punctures shallow. WH 0.96x LH; WF 0.48x WH, WF 1.0x HE, DAO 0.18x WF, OOL $0.74 \mathrm{x}$ WOT, frontal angle of ocellar triangle compact slightly acute, posterior ocellus distant from vertex crest 0.86x DAO, anterior margin of anterior ocellus nearly reaching eye top imaginary line. Vertex slightly convex, corner rounded. VOL $0.53 \mathrm{x}$ HE. Notaulus absent. Propodeal disc $0.86 \mathrm{x}$ as wide as long, $0.73 \mathrm{x}$ as high as long, median carina incomplete, basal triangle undefined, disc otherwise polished and weakly coria- ceous, posterior carina absent; declivity weakly coriaceous; lateral of propodeum nearly polished. Mesopleuron with weakly coriaceous callus. Fore wing without any trace of discoidal vein. Metasoma missing.

\section{Pseudisobrachium paraguayense (Kieffer, 1904)} Figs 295-297

Cotype, male. [PARAGUAY], Pto. 14 de Mayo, X.1896, G. Boggiani col. (MCSN). The "cotype" specimen studied by Evans (1961, 1964).

Diagnosis. Male (Pseudisobrachium specimen). Length 2.85 $\mathrm{mm}$. Dark castaneous. Mandible with four apical teeth. Clypeus with trapezoidal median lobe. Antennal segment XI 1.5 as long as wide, pubescence subappressed, longer than half of the diameter of basal flagellomeres, with few long erect setae outstanding pubescence. Eye long-haired. Frons coriaceous, punctures large, shallow and dense. WH $0.84 \mathrm{x} \mathrm{LH}$, WF $0.61 \mathrm{x} \mathrm{WH}$, WF 1.09x HE, DAO 0.2x WF, OOL 0.63x WOT, posterior ocellus distant from vertex crest $0.8 \mathrm{x}$ DAO. Vertex convex. VOL $0.63 x$ HE. Notaulus absent. Parapsidal furrow complete. Propodeal disc $0.82 \mathrm{x}$ as wide as long, $0.57 \mathrm{x}$ as high as long, basal triangle small and undefined, median carina incomplete, posterior carina absent. Mesopleuron with coriaceous callus. Fore wing with discoidal vein unpigmented. Genitalia: paramere with ventral arm about $2.0 \mathrm{x}$ wider than dorsal, ventral ramus with inner margin produced and angled basally; aedeagus mesoconvex, base wide, its apex not reaching apex of dorsal arm of paramere, slightly curved downward, ventral surface much concave and densely hairy medially.

\section{Dissomphalus albipes (Kieffer, 1904) Comb. nov. and Nom. rev.}

Holotype male: [PARAGUAY], Pto. 14 de Mayo, X.1896, G. Boggiani col. (MCSN).

Diagnosis. Male (Dissomphalus specimens). Length 2.23 $\mathrm{mm}$. Black. Mandible with three apical teeth. Clypeus with tridentate median lobe. Front coriaceous, with large and shallow punctures. Vertex slightly convex. Metasoma with tergite II vertical, with large and deep depression, their margins sharpened, except anterior, rather narrow and elongated, occupying median third of width of tergite and more than anterior half, reaching anterior margin, with elliptical lateral hairy tuft, separated of another by more than own small diameter, hairs directed slightly toward each other. Genitalia: paramere with apex slightly arched inward; volsella with cuspis much elongate and curved; aedeagus with ventral ramus shorter than dorsal body, wide, narrowing slightly to acute apex, ventral surface convex; dorsal body of aedeagus with two pairs of apical lobes; inner pair shorter than ventral ramus and semicircular, rounded portion downward, which is much produced, laminar and divergent dorsally with inner surface dense hairy, margin serrated with small denticles, outer pair long. 


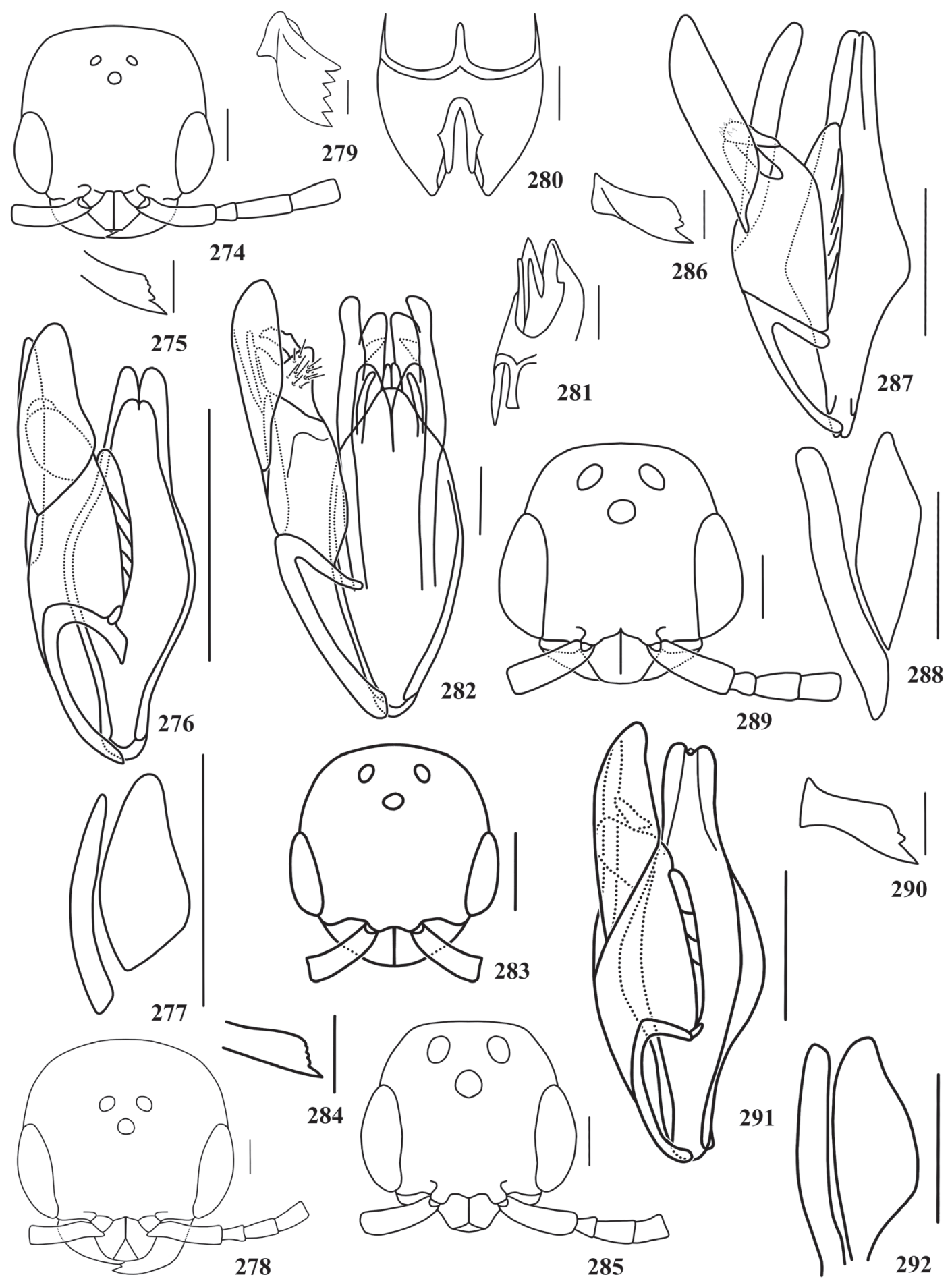

Figures 274-292. Pseudisobrachium opimum holotype male: (274) head, dorsal; (275) mandible, frontal; (276) genitalia, ventral; (277) paramere, full; P. optimum holotype male: (278) head, dorsal; (279) mandible, frontal; (280) hypopygium, ventral; (281) hypopygium, lateral; (282) genitalia, ventral; P. otiosum holotype male: (283) head, dorsal; (284) mandible, frontal; P. pallidipes holotype male: (285) head, dorsal; (286) mandible, frontal; (287) genitalia, ventral; (288) paramere, full; P. argentinicum holotype male: (289) head, dorsal; (290) mandible, frontal; (291) genitalia, ventral; (292) paramere, full. Bar $=250 \mu \mathrm{m}$. 
Remarks: This species was originally described as Rhabdepyris (?) albipes by KIEFFER (1904). He tentatively classified this species as Rhabdepyris, pointing out that "cette espèce devra probablement former un genre à part," i.e., "this species shall probably represent a distinct genus," what explains the question mark next to the generic name. Later, KIEFFER (1914) created Parisobrachium to accommodate this species. He stated: "54. Gen. Parisobrachium nom. nov. Kieffer" and "1905 Rhabdepyris? (part), Kieffer in Ann. Mus. Genova, v. 41, p. 411." With this nomenclatural act, it is clear that Kieffer created a new genus rather than a new name as he stated. Parisobrachium was created as a monotypic genus, with Rhabdepyris (?) albipes as the only possibility for the type species. This species was described from one specimen from Paraguay, by KiefFer (1904). As far as I know, there is no evidence that the description was based on more than one specimen. In his paper, Kieffer described many species and apparently always indicated the number of studied specimens when there was more than one. Curiously, however, Evans (1961: 222) received a "cotype" of Rhabdepyris? albipes from Dr. Delfa Guiglia, then the curator of the Museo Civico di Storia Naturale G. Doria, and, based on it, designated Parisobrachium as new junior synonymy of Pseudisobrachium. Later, Evans (1964: 85) described this "cotype" and provided the new name Pseudisobrachium paraguayense, since the name albipes was preoccupied in Pseudisobrachium by a species described by ASHMEAD (1894).

When I visited the Museo Civico di Storia Naturale G. Doria in January 2004, the curator, Dr. Roberto Poggi, gave me "two syntypes" of Rhabdepyris ? albipes with identical labels, i.e., same place, date, collector and son on. I examined both specimens in great detail, trying to determine on which of them KiefFer (1904) based his description of Rhabdepyris (?) albipes. I concluded, however, that the specimen studied by Evans (1964) does not correspond to any specimen of the series type of Rhabdepyris? albipes studied by KIEFFER (1904). The specimen studied by Evans (1964) belongs to Pseudisobrachium and the "other syntype" is a Dissomphalus specimen. In fact, there are characters in Kieffer's original description that best fit Dissomphalus: body length, mandible and antenna color, length/breadth of the antennal segments, VOL/HE ratio, pronotum/mesoscutum length ratio, presence and disposition of the notauli and parapsidal furrows, width/length ratio for the propodeal disc, presence and disposition of the median and the discal carinae of the propodeal disc, and the wing venation, particularly the shape of the transverse vein. Besides, there are one red label written "TYPUS" and one second label handwritten "Rhabdepyris albipes K." At the same time, there is no identification label with the Pseudisobrachium specimen.

Considering such evidence, I am fully convinced that KIEFFER (1904) based the description of Rhabdepyris (?) albipes only on the Dissomphalus specimen rather than on Pseudisobrachium specimen. Based on this, I propose Parisobrachium as a new junior synonymy of Dissomphalus, transfer $P$. paraguayense to Dissomphalus, and re-establish its original name Dissomphalus albipes (Kieffer, 1904).

A descriptions for each of both specimens discussed here is presented below. The Pseudisobrachium specimen seems to correspond either to a new species or to the male of $P$. distinguendum, which was described in the same paper and based on a female also collected in Pto. 14 de Mayo, Paraguay, in X.1896 by G. Boggiani. It is very similar to P. minimum Evans and differs from it by having the aedeagus very wide. I judged most reasonable not make any nomenclatural act at this time, in order to avoid increasing the degree of taxonomic complexity of this case.

\section{Pseudisobrachium parcum Evans, 1969 Figs 298-301}

Holotype, male. CUBA, Soledad n[ea]r Cienfuegos, [Las Villas], Soledad, 6-20.VIII, Berlese funnel, N. A. Weber col. (MCZ).

Descriptions. Male. Length $2.7 \mathrm{~mm}$. Castaneous, head darker; fore wing hyaline. Mandible with five apical teeth, upper four small and nearly of equal size. Clypeus with trapezoidal median lobe. Antenna with segment XI 1.38x as long as wide, pubescence of basal flagellomeres subappressed, with some long erect setae outstanding pubescence. Eye bulging, long-haired. Frons coriaceous, punctures shallow. WH $0.94 \mathrm{x}$ LH; WF 0.53x WH, WF 0.85x HE, DAO 0.3x WF, OOL $0.54 \mathrm{x}$ WOT, posterior ocellus close to vertex crest, anterior margin of anterior ocellus nearly reaching eye top imaginary line. Vertex convex, corner rounded. VOL 0.65x HE. Notaulus absent. Propodeal disc $0.78 \mathrm{x}$ as wide as long, $0.57 \mathrm{x}$ as high as long, median carina incomplete, disc mostly nearly polished, posterior carina absent; declivity aciculate; lateral of propodeum polished. Mesopleuron with weakly coriaceous callus. Fore wing with discoidal vein weakly pigmented, interstitial with median vein. Genitalia: paramere with ventral arm $2.6 \mathrm{x}$ wider than dorsal; aedeagus mesoconvex, its apex reaching beyond apex of dorsal arm of paramere, base narrow.

\section{Pseudisobrachium pauxillum Evans, 1969} Figs 302-306

Pseudisobrachium retusum Evans, 1969. Syn. nov.

Holotype, male. ARGENT[INA], Tucumán, Horco Molle, San X\{J\}axier Mts., 10-31.VII.1966, L. Stange col. (FIML).

Descriptions. Male. Length $4.6 \mathrm{~mm}$. Black. Mandible with five apical teeth, subupper very small. Clypeus with trapezoidal median lobe, apical margin angularly concave, median carina bifurcated apically. Antennal segment XI 1.2x as long as wide, pubescence appressed, as long as half of diameter of basal flagellomeres, with some long suberect setae outstanding pubescence. Eye long-haired. Frons strongly coriaceous, punctures small. WH 0.97x LH, WF 0.65x WH, WF 1.37x HE, DAO 0.17x $\mathrm{WF}$, OOL 1.0x WOT, posterior ocellus distant from vertex crest 

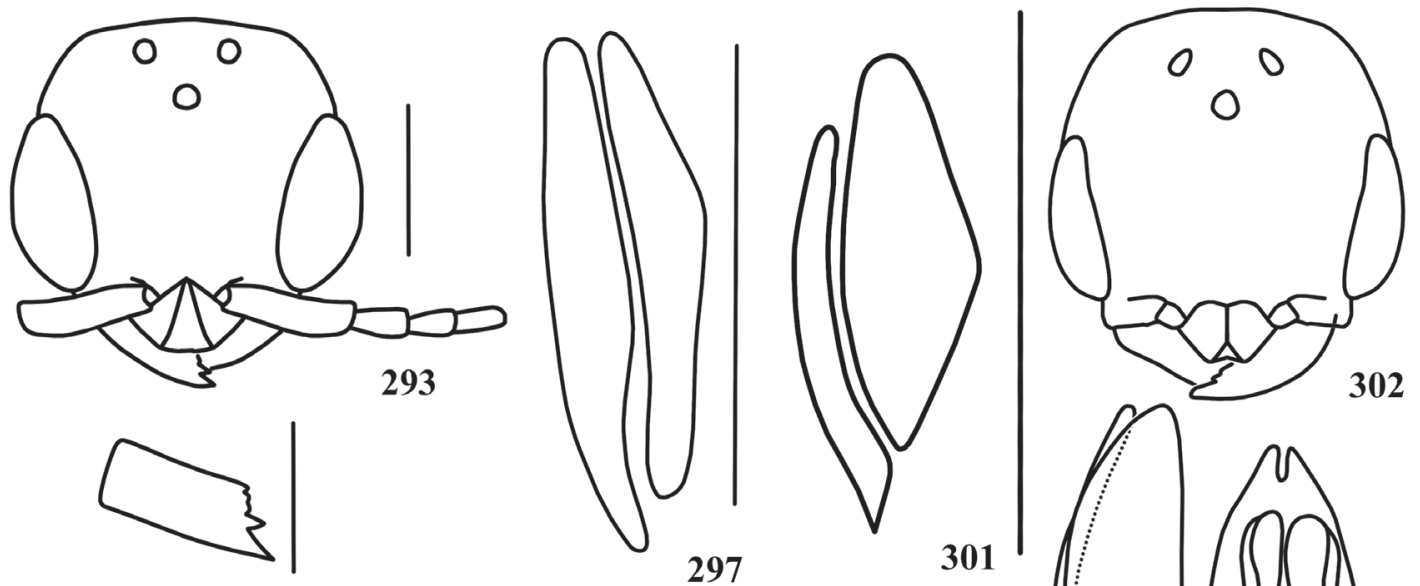

294
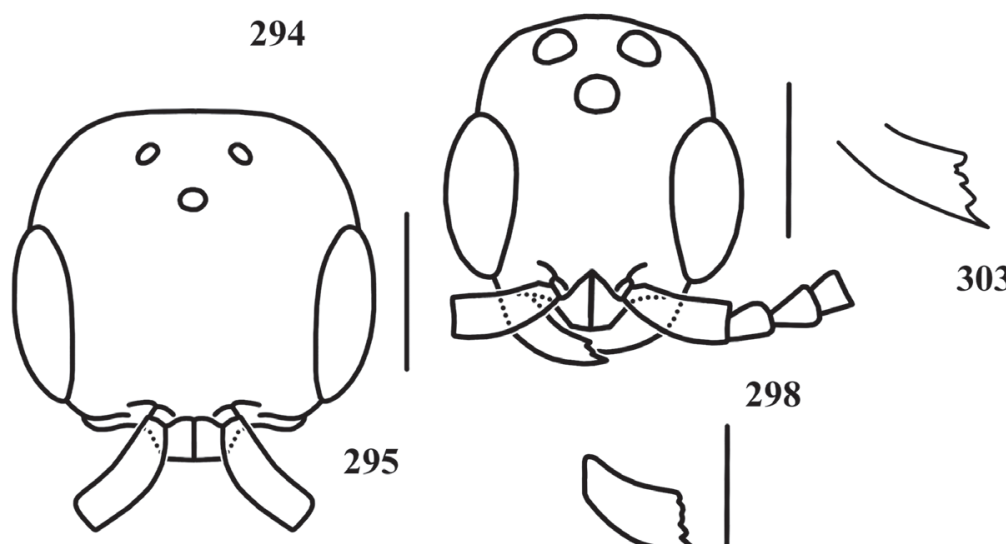

03
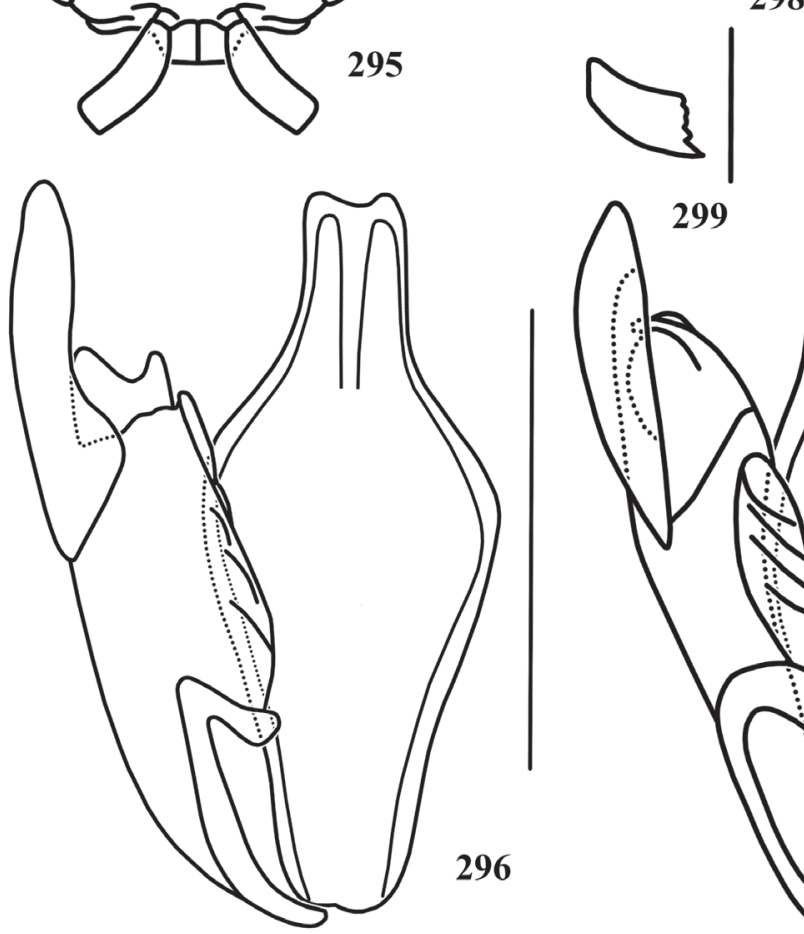

301
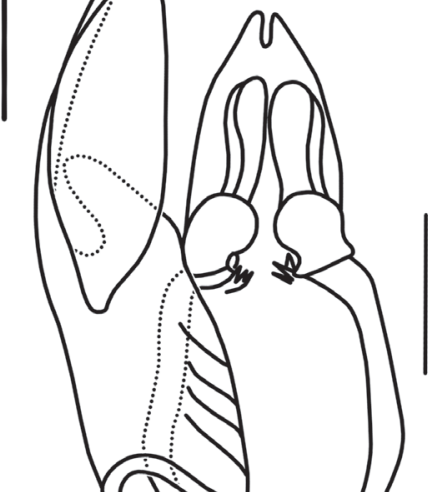
1.0x DAO. Vertex slightly convex, corner rounded. VOL $0.77 \mathrm{x}$ HE. Notaulus absent on posterior half of mesoscutum. Propodeal disc $1.24 \mathrm{x}$ as wide as long, $0.9 \mathrm{x}$ as high as long, basal triangle large, disc areolate posteriorly, median carina incomplete, posterior carina ill defined; declivity areolate. Mesopleuron with coriaceous large callus. Fore wing with discoidal vein badly pigmented basally, not interstitial with median vein. Hypopygium with posterior margin strongly concave. Genitalia: paramere with ventral arm $1.6 \mathrm{x}$ wider than strongly angled dorsal arm; aedeagus oval, its apex not reaching apex of dorsal arm of paramere, triangular, and emarginated, ventral margin with gibbous lobe, with basal denticles.

Remarks. The angled shape of the dorsal arm of the paramere and the bottle-shaped aedeagus with denticles on the gibbous lobe are unusual for this genus.

\section{Pseudisobrachium retusum Evans, 1969. Syn. nov.} Figs 307-311

Holotype, male. ARGENT[INA], Tucumán, Horco Molle, San X\{J\}axier Mts., 4-12.I.1966, L. Stange col. (FIML).

Descriptions. Male. Length $3.4 \mathrm{~mm}$. Dark castaneous. Mandible with five apical teeth, upper three teeth smaller than sublower. Clypeus with trapezoidal median lobe, apical margin angularly concave, median carina bifurcated apically. Antennal segment XI $1.6 \mathrm{x}$ as long as wide, pubescence subappressed, shorter than half of diameter of basal flagellomeres, with some long suberect setae outstanding pubescence. Eye long-haired. Frons strongly coriaceous, punctures inconspicuous. WH 1.07x LH, WF 0.61x WH, WF 1.27x HE, DAO 0.13x WF, OOL 1.0x WOT, posterior ocellus distant from vertex crest 1.6x DAO. Vertex slightly convex, corner rounded. VOL $0.71 \mathrm{x}$ HE. Temple subparallel anteriorly. Notaulus short anteriorly. Propodeal disc $1.1 \mathrm{x}$ as wide as long, $0.83 \mathrm{x}$ as high as long, basal triangle defined, median carina incomplete, posterior carina ill defined. Mesopleuron with callus ill defined. Fore wing with discoidal vein unpigmented, interstitial with median vein. Hypopygium with posterior margin strongly concave. Genitalia: paramere with ventral arm $1.7 \mathrm{x}$ wider than strongly angled dorsal arm; aedeagus oval, its apex not reaching apex of dorsal arm of paramere, triangular, and emarginated, ventral margin with gibbous lobe, with basal denticles.

Remarks. The hypopygium and the genitalia are absolutely similar to those of $P$. pauxillum. The general features of both species are very similar to each other and I propose it as a new junior synonym for $P$. pauxillum.

\section{Pseudisobrachium perpunctatum Evans, 1961} Figs $312-315$

Holotype, male. MEX[ICO], Oax[aca], Tehuantepec, 200', 9.VIII.1958, E. G. Matthews col. (MCZH, \#30266).

Descriptions. Male. Length $4.9 \mathrm{~mm}$. Dark castaneous; fore wing subhyaline. Mandible with five apical teeth, upper three smaller than sublower. Clypeus with trapezoidal median lobe, median carina bifurcated apically. Antenna with segment XI $1.29 \mathrm{X}$ as long as wide, pubescence of basal flagellomeres appressed and short, with some long erect setae outstanding pubescence. Eye long-haired. Frons polished, with dense shallow punctures. WH 0.97x LH; WF 0.6x WH, WF 1.12x HE, DAO 0.19x WF, OOL $0.86 \mathrm{x}$ WOT, posterior ocellus close to vertex crest, anterior margin of anterior ocellus closed to eye top imaginary line. Vertex slightly convex, corner rounded. VOL $0.64 x$ HE. Notaulus present on anterior third of mesoscutum. Propodeal disc $0.86 \mathrm{x}$ as wide as long, $0.57 \mathrm{x}$ as high as long, median carina incomplete, basal triangle defined, disc with posterior polished area, posterior carina indistinguishable from strigulation; declivity and lateral of propodeum aciculate. Mesopleuron with polished callus. Fore wing with discoidal vein unpigmented, interstitial with median vein. Genitalia: paramere with ventral arm $1.7 x$ wider than dorsal; aedeagus mesoconvex, its apex not reaching apex of dorsal arm of paramere, ventral surface with three pairs of teeth directed inward.

\section{Pseudisobrachium petiolatum Evans, 1961 Figs 316-318}

Holotype, male. PANAMA, Canal Zone, Tabernilla, 27.IV.[19]07, A. Busck col. (USNM, \#65385). Genitalia slide \#HE375.

Descriptions. Male. Length $2.81 \mathrm{~mm}$. Dark castaneous. Mandible with five apical teeth, upper three teeth smaller than sublower. Clypeus with subtrapezoidal median lobe. Antenna thick, segment XI 1.78 as long as wide, pubescence of basal flagellomeres appressed, with some erect setae outstanding pubescence. Eye long-haired. Frons weakly coriaceous, punctures small. WH 0.92x LH, WF 0.59x WH, WF 1.0x HE, ocelli bulging, DAO 0.12x WF, OOL 1.0x WOT, posterior ocellus distant from vertex crest $0.4 \mathrm{x}$ DAO. Vertex broadly convex. VOL $0.68 x$ HE. Temple strongly diverging anterad. Notaulus on anterior half of mesoscutum, inconspicuous. Propodeal disc $0.78 \mathrm{x}$ as wide as long, $0.63 \mathrm{x}$ as high as long, median carina incomplete, weakly rugulose anteriorly, posterior carina absent. Mesopleuron with weakly coriaceous callus. Fore wing with discoidal vein tubular for very short distance, arising well down on TMV. Metasoma petiolate. Genitalia: paramere with ventral arm slightly wider than dorsal; aedeagus mesoconvex, apex slightly not reaching apex of dorsal arm of paramere.

\section{Pseudisobrachium plaumanni Evans, 1964} Figs 319-321

Holotype, male. BRAZIL, [Santa Catarina], Nova Teutonia, $27^{\circ} 11^{\prime}$ B 52²3'L, 17.XII.1937, Fritz Plaumann col., B.M. 1937424 (BMNH, \#13369).

Descriptions. Male. Length $5.5 \mathrm{~mm}$. Black, anterior half of pronotal disc testaceous. Mandible with five apical teeth, upper three teeth smaller than sublower. Clypeus with sub- 

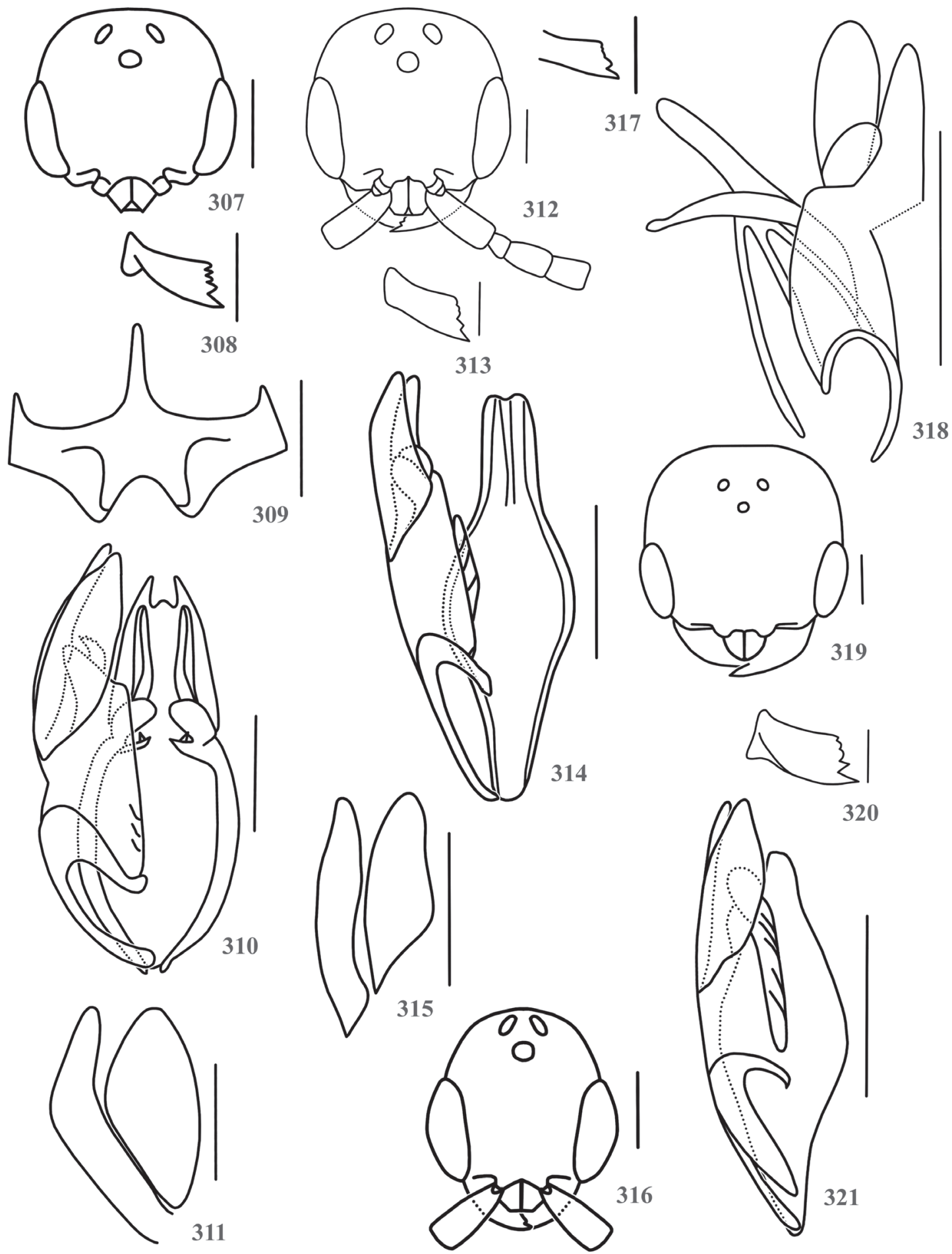

Figures 307-321. Pseudisobrachium retusum: holotype male: (307) head, dorsal; (308) mandible, frontal; (309) hypopygium, ventral; (310) genitalia, ventral; (311) paramere, full; P. perpunctatum holotype male: (312) head, dorsal; (313) mandible, frontal; (314) genitalia, ventral; (315) paramere, full; P. petiolatum holotype male: (316) head, dorsal; (317) mandible, frontal; (318) genitalia, lateral; P. plaumanni holotype male: (319) head, dorsal; (320) mandible, frontal; (321) genitalia, ventral. Bar = $250 \mu \mathrm{m}$. 
rounded median lobe, median carina high and arched in profile. Antennal segment XI 2.0x as long as wide, pubescence of basal flagellomeres subappressed/suberect with few setae erect outstanding pubescence. Frons polished, punctures small. WH 0.97x LH, WF 0.69x WH, WF 1.53x HE, DAO 0.08x WF, OOL $1.57 \mathrm{x}$ WOT, posterior ocellus distant from vertex crest $2.29 \mathrm{x}$ DAO. Vertex convex, corner rounded. VOL 1.24x HE. Anterior half of pronotal disc with distinct coarse rugulation. Notaulus absent. Propodeal disc $0.83 \mathrm{x}$ as wide as long, $0.53 \mathrm{x}$ as high as long, median carina almost complete, disc entirely rugulose, posterior carina indistinguishable from rugulation. Mesopleuron with small callus. Fore wing with discoidal vein pigmented, interstitial with median vein. Genitalia: paramere with ventral arm $1.5 \mathrm{x}$ wider than dorsal; aedeagus mesoconvex, its apex not reaching apex of dorsal arm of paramere.

\section{Pseudisobrachium porteri Evans, 1966}

Figs 322-325

Holotype, male. PERU, [Cusco], Machu Picchu, 1900 m, 4-19.IX.[19]64, C. C. Porter col. (MCZH, \#31244).

Descriptions. Male. Length $4.6 \mathrm{~mm}$. Black; fore wing weakly subhyaline. Mandible with five apical teeth, uppermost tooth wide, upper three smaller than sublower. Clypeus with rectangular median lobe, lateral margin concave, apical margin somewhat biconvex. Antenna with segment XI about 2.0x as long as wide, pubescence of basal flagellomeres appressed, with some long erect setae outstanding pubescence; sensilla elongate and dense. Eye small, long-haired. Frons polished, punctures small. WH 0.94x LH; WF 0.69x WH, WF 1.57x HE, DAO 0.12x WF, OOL 1.5x WOT, ocellar triangle compact with acute frontal angle, posterior ocellus distant from vertex crest 2.16x DAO. Vertex convex, corner broadly rounded. VOL 1.2x HE. Notaulus present on anterior third of mesoscutum. Propodeal disc $0.91 \mathrm{x}$ as wide as long, 0.66x as high as long, median carina incomplete, basal triangle large, disc otherwise strigulate, posterior carina absent; declivity aciculate; lateral of propodeum coriaceous-aciculate. Mesopleuron with polished callus. Fore wing with discoidal vein pigmented, not tubular, not interstitial with median vein. Genitalia: paramere with ventral arm 1.6x wider than dorsal; aedeagus mesoconvex, its apex not reaching apex of dorsal arm of paramere, wider than base.

\section{Pseudisobrachium praecipuum Evans, 1969}

Figs 326-329

Holotype, male. JAMAICA, 4000 feet, Hardwar Gap, 6.VII.1966, Howden \& Becker col. (CNCI, \#10994).

Descriptions. Male. Length $3.5 \mathrm{~mm}$. Black. Mandible with four apical teeth, upper three small and nearly of equal size. Clypeus with broad trapezoidal median lobe, apical margin barely concave, median carina bifurcated apically. Antennal segment XI about 1.7 as long as wide, pubescence of basal flagellomeres long and suberect, with some longer erect setae outstanding pubescence. Frons somewhat strongly coriaceous, punctures small. WH 1.03x LH, WF 0.7x WH, WF 1.65x HE, DAO $0.13 \mathrm{x}$ WF, OOL $1.8 \mathrm{x}$ WOT, posterior ocellus distant from vertex crest $1.8 \mathrm{x}$ DAO. Vertex slightly convex, corner rounded. VOL 0.96x HE. Notaulus well impressed, present on anterior half of mesoscutum. Propodeal disc $0.93 \mathrm{x}$ as wide as long, $0.67 \mathrm{x}$ as high as long, median carina incomplete, posterior carina absent. Mesopleuron with polished callus. Fore wing with discoidal vein very weakly pigmented, not interstitial with median vein. Genitalia: paramere with ventral arm $1.6 x$ wider than dorsal arm; aedeagus mesoconvex, its apex nearly aligned with apex of dorsal arm of paramere.

\section{Pseudisobrachium pseudoobscurum Evans, 1969 Figs 330-333}

Holotype, male. JAMAICA, 4000 feet, Hardwar Gap, 13.VII.1966, Howden \& Becker col. (CNCI, \#10995).

Descriptions. Male. Length $2.7 \mathrm{~mm}$. Dark castaneous. Mandible with three apical teeth, upper two small and nearly of equal size. Clypeus with subtrapezoidal median lobe, apical margin straight, median carina bifurcated apically. Antennal segment XI about 2.0 as long as wide, pubescence of basal flagellomeres long and subappressed, with some longer erect setae outstanding pubescence. Eye large and bulging. Frons coriaceous, punctures small. WH 1.04x LH, WF 0.57x WH, WF $1.05 x$ HE, ocelli large, DAO $0.24 x$ WF, OOL only $0.73 x$ WOT, posterior ocellus distant from vertex crest $0.43 \mathrm{x}$ DAO, anterior margin of anterior ocellus nearly reaching eye top imaginary line. Vertex slightly convex. VOL $0.55 x$ HE. Temple strongly divergent anterad. Notaulus present on anterior half of mesoscutum. Propodeal disc as wide as long, $0.67 \mathrm{x}$ as high as long, median carina present on anterior half, posterior carina absent. Mesopleuron with polished callus. Fore wing with discoidal vein weakly pigmented, not interstitial with median vein. Genitalia: paramere with ventral arm $2.8 \mathrm{x}$ wider than dorsal arm; aedeagus mesoconvex, its apex reaching beyond apex of dorsal arm of paramere.

\section{Pseudisobrachium pulcherrimum Evans, 1964 Figs 334-337}

Holotype, male. C[OSTA] R[ICA], Turrialba, 17.II.[19]49 (USNM, \#70056). Genitalia slide \#HE498.

Descriptions. Male. Length $3.71 \mathrm{~mm}$. Dark castaneous, head black. Mandible with five apical teeth, upper three smaller than sublower. Clypeus with trapezoidal median lobe, apical margin straight, median carina bifurcated apically. Antenna thick, segment XI 2.38 as long as wide, pubescence of basal flagellomeres appressed, with few erect setae outstanding pubescence. Eye long-haired. Frons polished, densely punctuated. WH 0.98x LH, WF 0.68x WH, WF 1.5x HE, DAO 0.12x WF, ocellar triangle compact, OOL 1.79x WOT, posterior ocellus distant from vertex crest $1.8 \mathrm{x}$ DAO. Vertex slightly convex, 


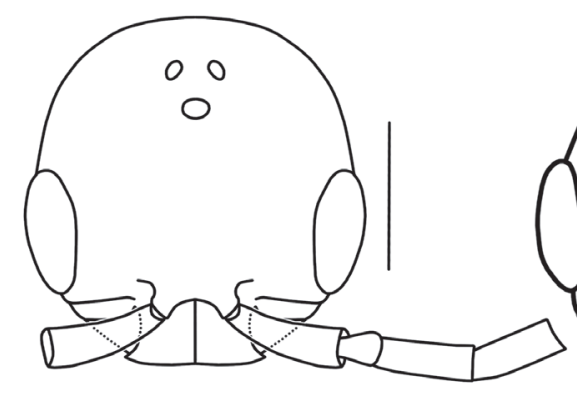

322

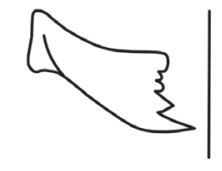

323
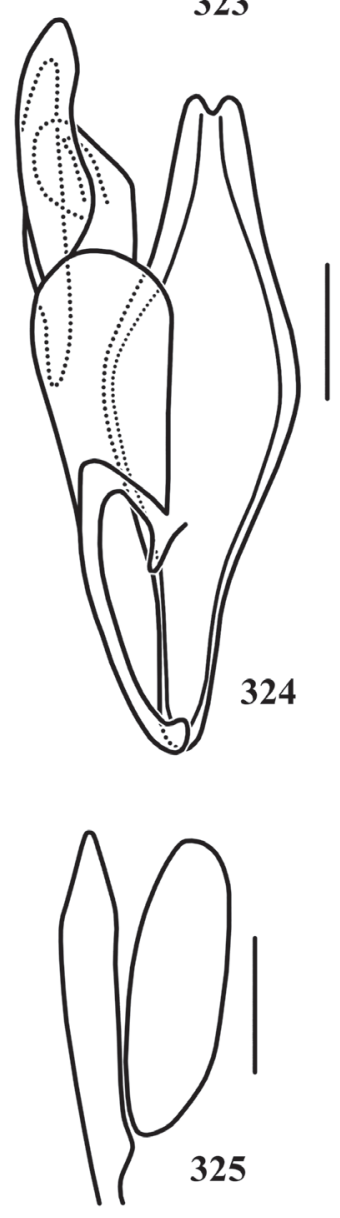

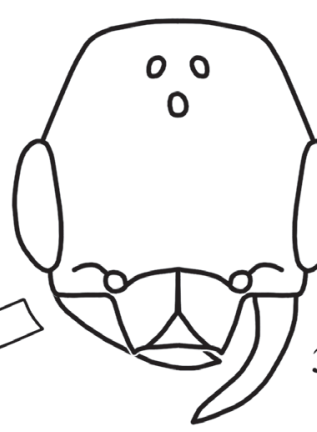

\section{6}

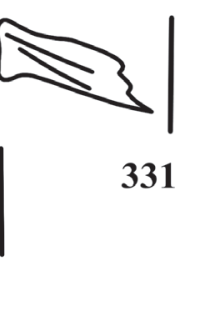

331

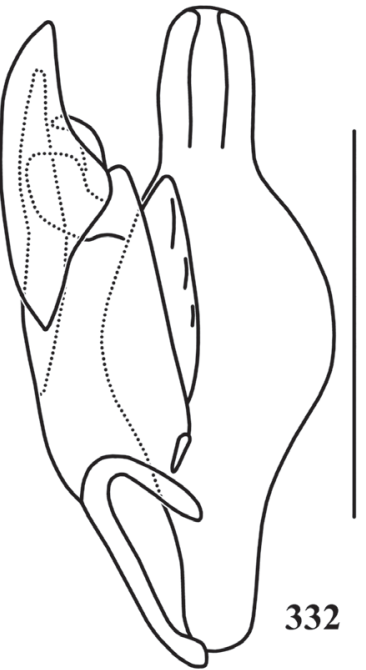

327
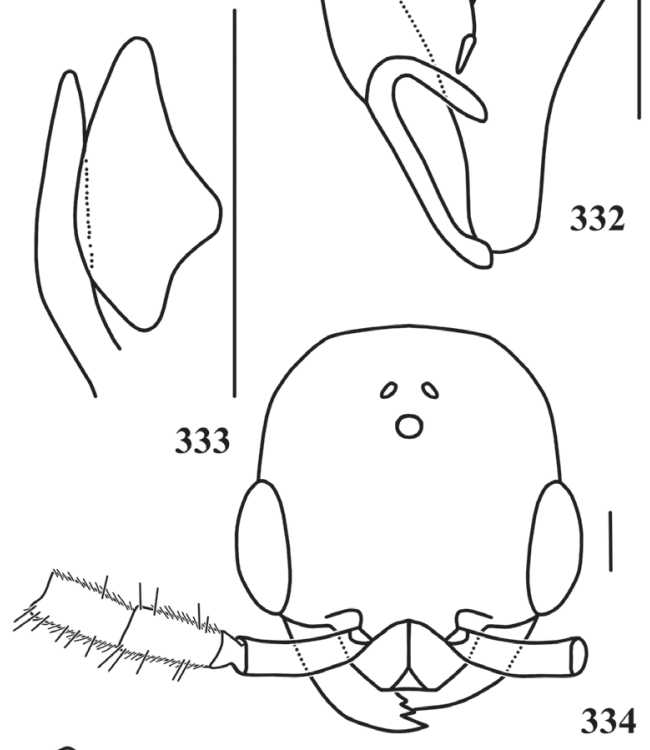

328

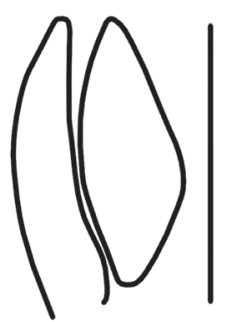

329

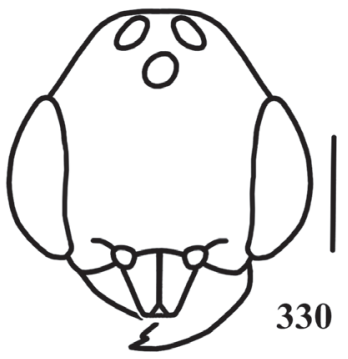

330
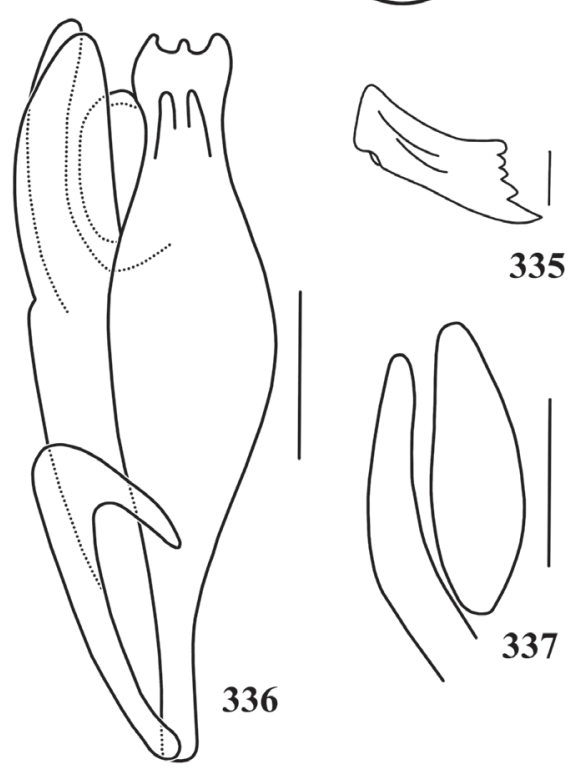

Figures 322-337. Pseudisobrachium porteri holotype male: (322) head, dorsal; (323) mandible, frontal; (324) genitalia, ventral; (325) paramere, full; P. praecipuum holotype male: (326) head, dorsal; (327) mandible, frontal; (328) genitalia, ventral; (329) paramere, full; P. pseudoobscurum holotype male: (330) head, dorsal; (331) mandible, frontal; (332) genitalia, ventral; (333) paramere, full; P. pulcherrimum holotype male: (334) head, dorsal; (335) mandible, frontal; (336) genitalia, ventral; (337) paramere, full. Bar $=250 \mu \mathrm{m}$. 
corner rounded. VOL 1.16x HE. Notaulus on anterior half of mesoscutum. Propodeal disc $1.05 \mathrm{x}$ as wide as long, $0.78 \mathrm{x}$ as high as long, median carina nearly complete, strongly reticulate, posterior carina indistinguishable; declivity strigulate. Mesopleuron with polished callus. Fore wing with discoidal vein weakly pigmented basally, interstitial with median vein. Genitalia: paramere with ventral arm 2.0x wider than dorsal; aedeagus mesoconvex, apex tridentate, slightly not reaching apex of dorsal arm of paramere.

\section{Pseudisobrachium rapoporti Ogloblin, 1963} Figs 338-339

Holotype, female. ARGENTINA, Buenos Aires, cerca de Bahia Blanca, salina Grünbein, 10.XII.1961, E. H. Rapoport col. (Ogloblin's collection, not found).

Descriptions. Female. Length $3.6 \mathrm{~mm}$. Castaneous. Mandible with three apical teeth, lowermost large. Clypeus with trapezoidal median lobe, apical margin convex. Eye absent. Frons weakly coriaceous, punctures large. Head with sides nearly straight and subparallel, head wider anterior, vertex nearly straight, $1.2 \mathrm{x}$ as long as wide. Pronotal disc, mesoscutum and propodeal disc in ratio of 5:3:4. Pronotal disc with concave side. Mid tibia strongly spinose.

Remarks. The descriptions and the illustration are extracted on the original publication.

\section{Pseudisobrachium rettenmeyeri Evans, 1961}

Figs 340-343

Holotype, male. PANAMA, [Panama], C[anal] Z[one], Barro Colorado I[slan]d, 28.III.1955, \#1065, C. Rettenmeyer col. (SEMC).

Descriptions. Male. Length $4.5 \mathrm{~mm}$. Dark castaneous; fore wing subhyaline. Mandible with five apical teeth, uppermost wide, upper three smaller than sublower. Clypeus with trapezoidal median lobe. Antenna with segment XI about $1.5 \mathrm{x}$ as long as wide, pubescence appressed and about as long as half diameter of basal flagellomeres, with some erect setae outstanding pubescence. Eye long-haired. Frons somewhat strongly coriaceous, punctures inconspicuous. WH 0.98x LH; WF 0.69x WH, WF 1.45x HE, DAO 0.12x WF, OOL 1.51x WOT, frontal angle of ocellar triangle acute, posterior ocellus distant from vertex crest 2.0x DAO. Vertex slightly convex. VOL 0.93x HE. Pronotal disc short. Notaulus complete. Propodeal disc $1.0 \mathrm{x}$ as wide as long, $0.66 \mathrm{x}$ as high as long, basal triangle undefined, median carina incomplete, mostly finely strigulate, posterior carina indistinguishable; declivity aciculate; lateral of propodeum weakly coriaceous. Mesopleuron with polished callus. Fore wing with discoidal vein weakly pigmented basally, interstitial with median vein. Genitalia: paramere wide, ventral margin angulate, with ventral arm $2.0 \mathrm{x}$ wider than dorsal; aedeagus mesoconvex, much dilated, apex wide, not reaching apex of dorsal arm of paramere.

\section{Pseudisobrachium rotundum Waichert \& Azevedo, 2004}

\section{Figs 344-347}

Holotype, male. BRAZIL, E[spírito] S[anto], Cariacica, Res[erva] Biol[ógica de] Duas Bocas, 26.XII.1996, sweeping, C.O. Azevedo col. (UFES).

Descriptions. Male. Length $3.8 \mathrm{~mm}$. Black; fore wing subhyaline. Mandible with five apical teeth, upper three teeth smaller than sublower. Clypeus with rounded median lobe, median carina arched in profile, bifurcated apically. Antennal segment XI 2.2x as long as wide; pubescence of basal flagellomeres subappressed, with some erect setae outstanding pubescence. Frons strongly coriaceous, punctures inconspicuous. WH 1.04x LH, WF 0.63x WH, WF 1.32x HE; DAO 0.14x $\mathrm{WF}$, OOL 1.37x WOT, posterior ocellus distant from vertex crest $1.45 x$ DAO. Vertex nearly straight. VOL $0.67 x$ HE. Temple divergent anterad. Pronotal disc short, with anterior transverse elevation. Notaulus absent. Propodeal disc $1.33 \mathrm{x}$ as wide as long, $1.0 \mathrm{x}$ as high as long, basal triangle large, median carina incomplete, posterior carina absent; declivity irregularly strigulate; lateral of propodeum mostly coriaceous. Mesopleuron with polished callus. Fore wing with discoidal vein weakly pigmented, nearly interstitial with median vein. Genitalia: paramere with ventral arm $2.4 \mathrm{x}$ wider than dorsal arm; aedeagus mesoconvex, constricted subapically, apex emarginated.

Remarks. The genitalia are in poor condition.

\section{Pseudisobrachium rufopictum (Kieffer, 1910) Figs 348-351 \\ Holotype, male. BRAZIL, Pará, Baker col., \#6792 (CASC,} \#9734).

Descriptions. Male. Length $6.8 \mathrm{~mm}$. Black, metasoma bright light castaneous. Mandible broad apically, with five large of equal size apical teeth. Clypeus with trapezoidal median lobe, apical margin straight. Antennal segment XI $2.5 \mathrm{x}$ as long as wide, pubescence coarse, subappressed, much longer than half of diameter of basal flagellomeres, with some long erect setae outstanding pubescence. Eye bulging. Frons coriaceous, punctures small. WH 1.07x LH, WF 0.58x WH, WF 1.04x HE, DAO $0.18 \mathrm{x}$ WF, ocellar triangle very compact, OOL 1.0x WOT, posterior ocellus distant from vertex crest $1.78 \mathrm{x}$ DAO, posterior margin of anterior ocellus reaching eye top imaginary line. Vertex slightly convex. VOL $0.57 x$ HE. Notaulus conspicuous, convergent and incomplete posteriorly. Propodeal disc $1.05 \mathrm{x}$ as wide as long, $0.7 \mathrm{x}$ as high as long, median carina incomplete, strigulate, posterior carina indistinguishable; declivity strigulate. Mesopleuron with polished callus. Fore wing with discoidal vein weakly pigmented basally, interstitial with median vein. Genitalia: paramere with ventral arm $2.5 \mathrm{x}$ wider than dorsal arm; aedeagus mesoconvex, its apex slightly not reaching apex of dorsal arm of paramere.

Remarks. The notauli of this species are unusually conspicuous. 

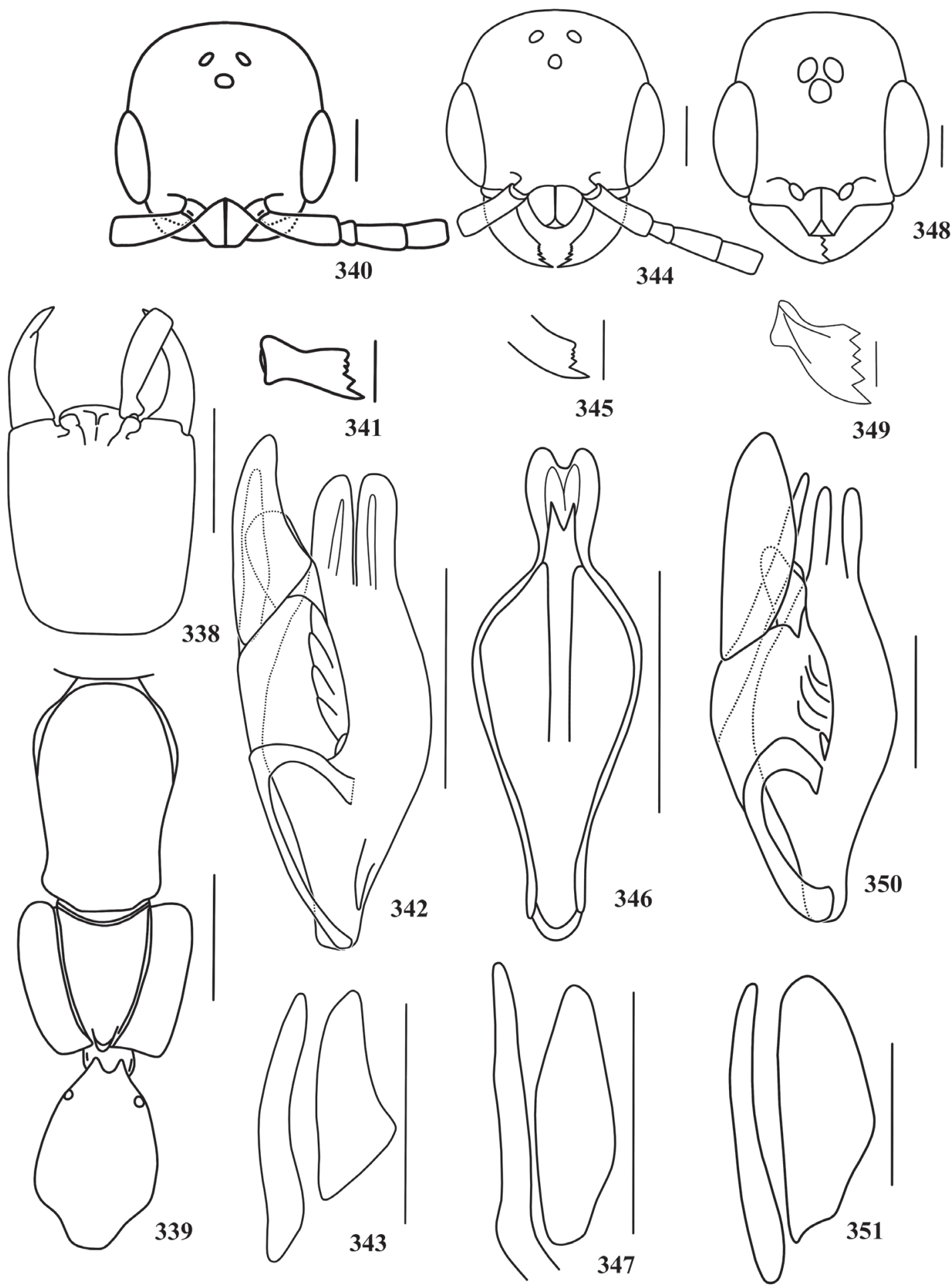

Figures 338-351. Pseudisobrachium rapoporti female, original illustration: (338) head, dorsal; (339) mesosoma, dorsal; P. rettenmeyeri holotype male: (340) head, dorsal; (341) mandible, frontal; (342) genitalia, ventral; (343) paramere, full; P. rotundum holotype male: (344) head, dorsal; (345) mandible, frontal; (346) genitalia, ventral; (347) paramere, full; P. rufopictum holotype male: (348) head, dorsal; (349) mandible, frontal; (350) genitalia, ventral; (351) paramere, full. Bar $=250 \mu \mathrm{m}$. 


\section{Pseudisobrachium solenopsidicola Bruch, 1917}

Figs 352-353

Syntypes female (two specimens, one lost) ARGENTINA: Sierra de la Ventana, ex. nest of Solenopsis saevissima tri'cuspis Forel, II.1916 (MLPA, not found)

Descriptions. Female. Length $3.5 \mathrm{~mm}$. Head and metasoma black, mesosoma dark castaneous. Mandible with four apical teeth, upper three in oblique series. Clypeus with median carina. Eye very small. Head quadrate, sides slightly convex and parallel, vertex slightly concave, about as long as wide. Mesosoma weakly coriaceous. Pronotal disc, mesoscutum and propodeal disc in ratio of 15:11:11. Pronotal disc with convex side. Mid tibia spinose. Metasoma not petiolate.

Remarks. The descriptions and the illustration are extracted on the original publication.

\section{Pseudisobrachium solenopsiphilum Ogloblin, 1963 Figs 334-355}

Syntype female (two specimens): ARGENTINA, Misiones, Loreto, 15.II.1932 and 12.XII.1933, collected at ant nest of Solenopsis (Ogloblin's collection, not found).

Descriptions. Female. Length $2.4 \mathrm{~mm}$. Castaneous. Mandible with four apical teeth. Clypeus with trapezoidal median lobe, apical margin convex. Eye with one facet. Malar space 5.0x longer than eye length. Frons with large punctures, median longitudinal band without punctures. Head with sides slightly convex, diverging anteriorly, vertex nearly straight, $1.3 \mathrm{x}$ as long as wide. Pronotal disc, mesoscutum and propodeal disc in ratio of 23:20:17. Pronotal disc with concave side. Mid tibia spinose.

Remarks. The descriptions and the illustration are extracted on the original publication.

\section{Pseudisobrachium stangei Evans, 1969 Figs 356-359}

Holotype, male. ARGENTINA, Tucumán, Horco Molle, San X\{J\}axier Mts., 3-10.IV.1966, L. Stange col. (FIML).

Descriptions. Male. Length $4.0 \mathrm{~mm}$. Dark castaneous, head nearly black. Mandible with four apical teeth, uppermost broad, upper four nearly of equal size. Clypeus with trapezoidal median lobe, apical margin straight. Antennal segment XI 1.7x as long as wide, pubescence subappressed, as long as half of diameter of basal flagellomeres, with some long suberect setae outstanding pubescence. Eye long-haired. Frons strongly coriaceous, with shallow and large punctures. WH $0.94 \mathrm{x} \mathrm{LH}$, WF $0.7 \mathrm{x}$ WH, WF 1.58x HE, DAO 0.17x WF, OOL 1.27x WOT, posterior ocellus distant from vertex crest 1.0x DAO. Vertex slightly convex, corner rounded. VOL 1.0x HE. Notaulus absent on posterior half of mesoscutum. Propodeal disc $0.77 \mathrm{x}$ as wide as long, $0.5 \mathrm{x}$ as high as long, basal triangle defined, median carina incomplete, posterior carina indistinguishable. Mesopleuron with polished callus. Fore wing with discoidal vein unpigmented basally, not interstitial with median vein. Genitalia: paramere with ventral arm 2.6x wider than dorsal; aedeagus mesoconvex, its apex not reaching apex of dorsal arm of paramere.

\section{Pseudisobrachium steinbachi Evans, 1966} Figs 360-363

Holotype, male. BOLIVIA, Santa Cruz, J. Steinbach col. (MCZH, \#31245).

Descriptions. Male. Length $6.2 \mathrm{~mm}$. Black; fore wing subhyaline. Mandible with five apical teeth, upper three smaller than sublower. Clypeus with trapezoidal median lobe, apical margin tridentate. Antenna with segment XI about 2.0x as long as wide, pubescence of basal flagellomeres appressed and short, with some erect setae outstanding pubescence. Eye small, longhaired. Frons polished, punctures small. WH 1.02x LH; WF 0.64x WH, WF 1.51x HE, DAO 0.15x WF, OOL 1.04x WOT, frontal angle of ocellar triangle right, posterior ocellus distant from vertex crest 2.6x DAO, anterior margin of anterior ocellus closed to eye top imaginary line. Vertex broadly convex. VOL 1.07x HE. Notaulus present on anterior half of mesoscutum. Propodeal disc $1.32 \mathrm{x}$ as wide as long, $0.9 \mathrm{x}$ as high as long, median carina incomplete, mostly strongly strigulate, posterior carina indistinguishable; declivity strongly strigulate; lateral of propodeum mostly polished. Mesopleuron with polished callus. Fore wing with discoidal vein spectral, not interstitial with median vein. Genitalia: paramere with ventral arm 1.9x wider than dorsal; aedeagus mesoconvex with base wide, its apex not reaching apex of dorsal arm of paramere, constricted below apex.

\section{Pseudisobrachium superbum Evans, 1961 Figs 364-366}

Holotype, male. PAN[AMA], Cano Saddle, Gatum L[ake], 17.V.[19]23, R. C. Shannon col. (USNM, \#65387). Genitalia slide \#HE374.

Descriptions. Male. Length $5.81 \mathrm{~mm}$. Dark castaneous, head black. Mandible with three large apical teeth in oblique series, upper two small and nearly of equal size. Clypeus with broad trapezoidal median lobe, apical margin straight, median carina bifurcated apically. Antenna thick, segment XI 2.2 as long as wide, pubescence of basal flagellomeres appressed and short, with few erect setae outstanding pubescence. Eye long-haired. Frons polished, punctures sparse. WH 0.84x LH, WF 0.67x WH, WF 1.08x HE, DAO 0.17x WF, ocellar triangle compact, OOL $1.07 \mathrm{x}$ WOT, posterior ocellus distant from vertex crest $0.89 \mathrm{x}$ $\mathrm{DAO}$, anterior margin of anterior ocellus nearly reaching eye top imaginary line. Vertex broadly convex. VOL $0.61 x$ HE. Notaulus wider posteriorly. Propodeal disc $1.03 \mathrm{x}$ as wide as long, $0.67 \mathrm{x}$ as high as long, median carina absent, basal triangle irregularly strigulate, disc otherwise transversely strigulate, posterior carina indistinguishable; declivity strigulate. Mesopleuron with large polished callus. Fore wing with discoidal vein unpigmented, interstitial with median vein. Genitalia: paramere with ventral arm $1.51 \mathrm{x}$ wider than dorsal; aedeagus mesoconvex, apex slender, not reaching apex of dorsal arm of paramere, base short. 


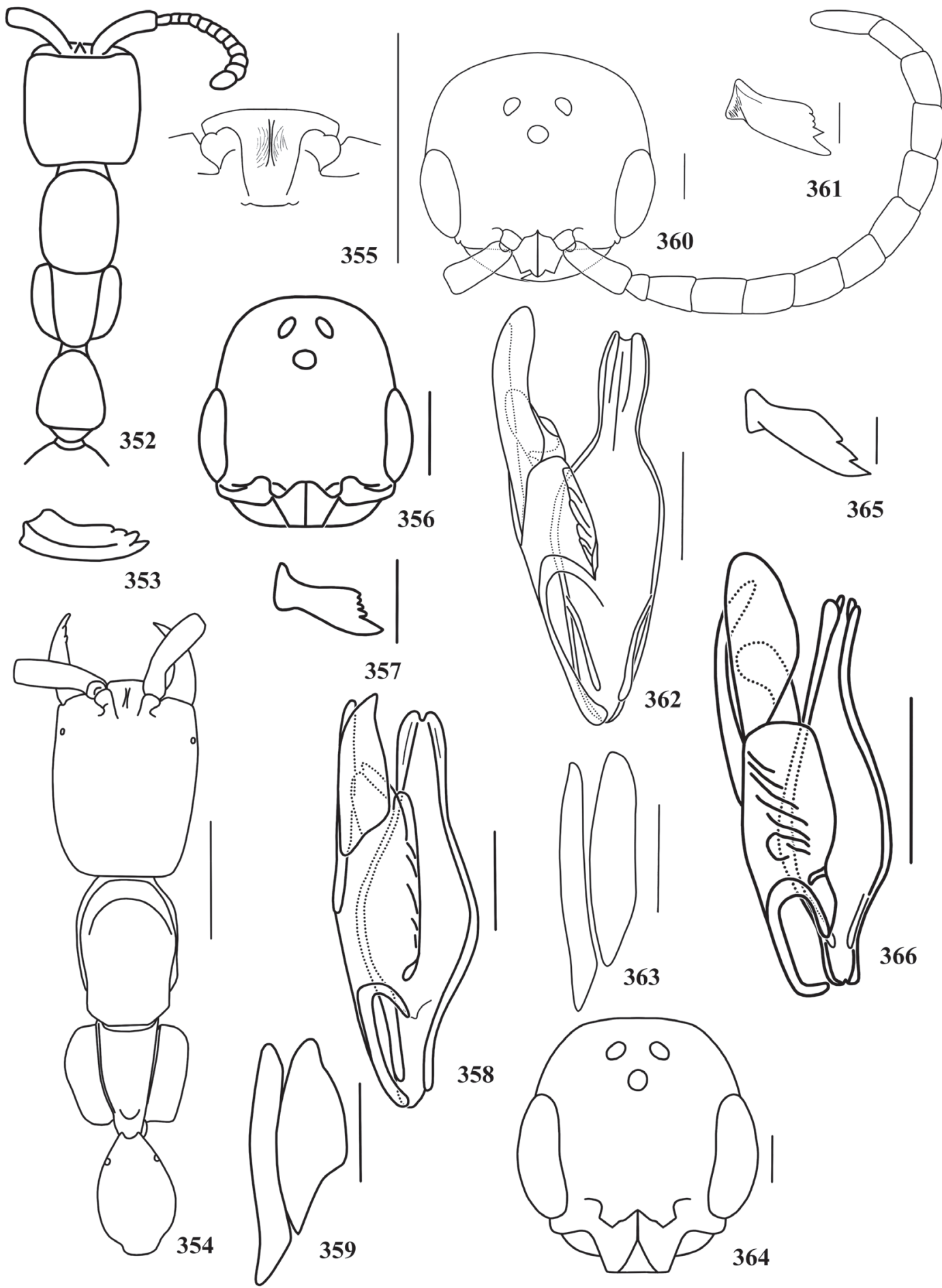

Figures 352-366. Pseudisobrachium solenopsidicola female: (352) head and mesosoma, dorsal; (353) mandible, frontal; P. solenopsiphilum original illustration, female: (354) head and mesosoma, dorsal; (355) clypeus, dorsal; P. stangei holotype male: (356) head, dorsal; (357) mandible, frontal; (358) genitalia, ventral; (359) paramere, full; P. steinbachi holotype male: (360) head, dorsal; (361) mandible, frontal; (362) genitalia, ventral; (363) paramere, full; P. superbum holotype male: (364) head, dorsal; (365) mandible, frontal; (366) genitalia, ventral. Bar $=250 \mu \mathrm{m}$. 


\section{Pseudisobrachium ternarium Evans, 1969}

Figs 367-370

Holotype, male. ARGENTINA, Tucumán, Horco Molle, Tuc, 3-10.IV.1966, L. Stange col. (FIML).

Descriptions. Male. Length $4.0 \mathrm{~mm}$. Dark castaneous, nearly black. Mandible with five apical teeth, upper three smaller than sublower. Clypeus with trapezoidal median lobe, apical margin badly convex, median carina bifurcated apically. Antennal segment XI 2.0x as long as wide, pubescence subappressed, as long as half of diameter of basal flagellomeres, with some long suberect setae outstanding pubescence. Eye long-haired. Frons coriaceous, with shallow and large punctures. WH 0.96x LH, WF 0.67x WH, WF 1.5x HE, DAO 0.18x WF, OOL 1.22x WOT, posterior ocellus distant from vertex crest $1.2 \mathrm{x}$ DAO. Vertex convex, corner rounded. VOL $0.63 x$ HE. Temple diverging. Notaulus very short anteriorly. Propodeal disc $0.91 \mathrm{x}$ as wide as long, $0.68 \mathrm{x}$ as high as long, basal triangle defined, disc rugulose, median carina complete, posterior carina indistinguishable. Mesopleuron with polished callus. Fore wing with discoidal vein weakly pigmented basally, interstitial with median vein. Genitalia: paramere with ventral arm $1.5 \mathrm{x}$ wider than dorsal; aedeagus mesoconvex, its apex about aligned with apex of dorsal arm of paramere.

\section{Pseudisobrachium terresi Mann, 1915}

Figs $371-372$

Holotype, female. HAITI, Petionville, W.M. Mann col. (USNM, \#65678)

Descriptions. Female. Length $6.3 \mathrm{~mm}$. Dark castaneous. Mandible with four nearly of equal size apical teeth in oblique series. Clypeus with trapezoidal median lobe, apical margin concave in dorsal view, triangular medially in frontal view. Eye light, subcircular, with one facet. Malar space about as long as eye length. Frons polished, punctures dense and conspicuous, with narrow unpunctuated longitudinal median band. Head with sides nearly straight and subparallel, head slightly wider anteriorly, vertex straight, $1.13 \mathrm{x}$ as long as wide. Pronotal disc, mesoscutum and propodeal disc in ratio of $\sim 5: 4: 4$. Mid tibia spinose. Metasoma not petiolate.

Remarks. The type is pinned together with one specimen of Formicidae.

\section{Pseudisobrachium testaceipes Kieffer, 1906}

Figs 373-375

Holotype, male. NICARAGUA, San Marcos, Baker col. \#69 (CASC, \#9756).

Descriptions. Male. Length $2.63 \mathrm{~mm}$. Dark castaneous. Mandible with five apical teeth, upper three teeth smaller than sublower. Clypeus with trapezoidal median lobe, apical margin barely concave. Antennal segment XI 1.16 as long as wide, pubescence subappressed, longer than half of diameter of basal flagellomeres, with some long erect setae outstanding pubescence. Frons coriaceous, punctures small and inconspicuous.
WH 1.0x LH, WF 0.69x WH, WF 1.58x HE, DAO 0.12x WF, OOL 1.38x WOT, posterior ocellus distant from vertex crest $1.14 \mathrm{x}$ DAO. Vertex slightly convex, corner rounded. VOL $0.8 \mathrm{x}$ HE. Notaulus very inconspicuous, present on anterior third of mesoscutum. Propodeal disc $1.0 \mathrm{x}$ as wide as long, $0.78 \mathrm{x}$ as high as long, median carina incomplete, posterior carina inconspicuous. Mesopleuron with coriaceous callus as remainder of areas of mesopleuron. Fore wing with discoidal vein unpigmented. Genitalia: paramere with ventral arm $2.75 x$ wider than dorsal arm; aedeagus mesoconvex, apex very wide, not reaching apex of dorsal arm of paramere.

\section{Pseudisobrachium transversum Evans, 1969 Figs 376-379}

Holotype, male. JAMAICA, 4000 feet, Hardwar Gap, 13.VII.1966, Howden \& Becker col. (CNCI, \#10988).

Descriptions. Male. Length $4.7 \mathrm{~mm}$. Black. Mandible with five apical teeth, upper three smaller than sublower. Clypeus with trapezoidal median lobe, apex narrow, apical margin nearly straight, median carina bifurcated apically. Antennal segment XI about 2.2 as long as wide, pubescence of basal flagellomeres subappressed, with some long erect setae outstanding pubescence. Frons strongly coriaceous, punctures large. WH $0.93 \mathrm{x}$ LH, WF 0.64x WH, WF 1.4x HE, DAO 0.15x WF, OOL 1.65x WOT, posterior ocellus distant from vertex crest $1.64 \mathrm{x}$ DAO. Vertex slightly convex, corner rounded, Temple divergent anterad. VOL 0.95x HE. Notaulus well impressed, present on anterior half. Propodeal disc strigulate, $0.95 \mathrm{x}$ as wide as long, $0.76 \mathrm{x}$ as high as long, median carina short, posterior carina absent. Mesopleuron with coriaceous callus. Fore wing with discoidal vein somewhat pigmented, not interstitial with median vein. Genitalia: paramere with ventral arm $2.5 \mathrm{x}$ wider than dorsal arm; aedeagus mesoconvex, apex wide, not reaching apex of dorsal arm of paramere.

\section{Pseudisobrachium triacutum Waichert \& Azevedo, 2004 Figs $380-385$}

Holotype, male. BRAZIL, E[spírito] S[anto], S[anta] Teresa, Est[ação] Biol[ógica de] Santa Lúcia, 23.VII.2001, sweeping, C.O. Azevedo \& R. Kawada col. (UFES).

Descriptions. Male. Length $3.2 \mathrm{~mm}$. Black; fore wing subhyaline. Mandible with five apical teeth, upper four small and nearly of equal size. Clypeus with trapezoidal median lobe, apical margin angularly concave, median carina bifurcated apically. Antennal segment XI 2.1x as long as wide; pubescence of flagellum subappressed, with few erect setae outstanding pubescence. Frons coriaceous, punctures shallow and large. $\mathrm{WH}$ 0.86x LH, WF 0.61x WH, WF 1.13x HE; DAO 0.2x WF; OOL 1.5x WOT, posterior ocellus distant from vertex crest $0.89 x$ DAO. Vertex convex. VOL $0.85 x$ HE. Temple divergent anterad. Notaulus very short and ill-defined. Propodeal disc $0.91 \mathrm{x}$ as wide as long, $0.51 \mathrm{x}$ as high as long, areolate anteriorly and strigulate posteriorly, median carina incomplete, lateral and posterior carinae ab- 

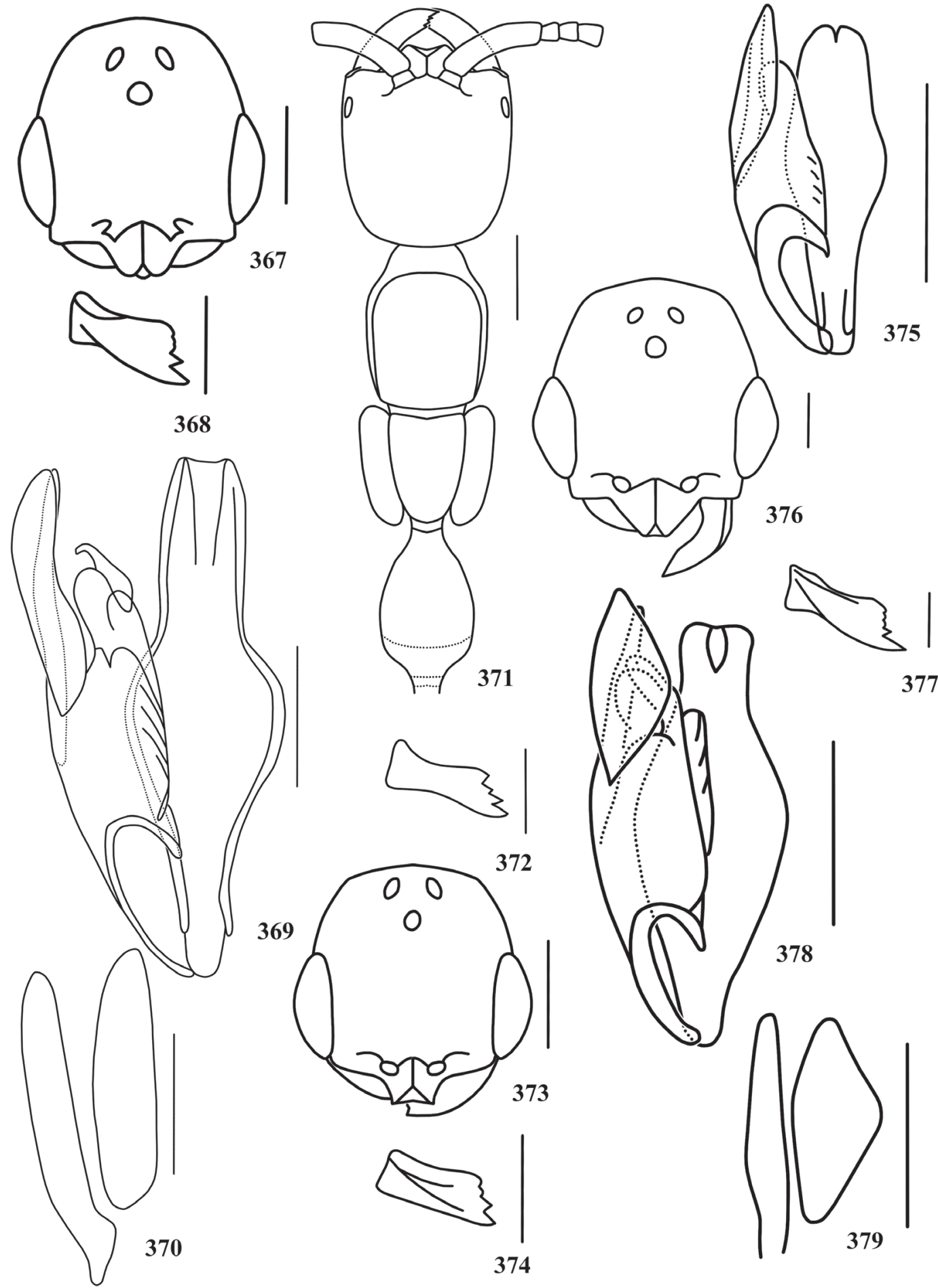

Figures 367-379. Pseudisobrachium ternarium holotype male: (367) head, dorsal; (368) mandible, frontal; (369) genitalia, ventral; (370) paramere, full; P. terresi holotype female: (371) head and mesosoma, dorsal; (372) mandible, frontal; P. testaceipes holotype male: (373) head, dorsal; (374) mandible, frontal; (375) genitalia, ventral; P. transversum holotype male: (376) head, dorsal; (377) mandible, frontal; (378) genitalia, ventral; (379) paramere, full. Bar $=250 \mu \mathrm{m}$. 
sent; declivity strigulate, lateral of propodeum weakly coriaceous. Mesopleuron coriaceous with coriaceous callus. Fore wing with discoidal vein nearly unpigmented, not interstitial with median vein. Hypopygium with posterior margin with angular emargination. Genitalia: paramere with ventral arm $2.0 \mathrm{x}$ wider than dorsal arm, aedeagus mesoconvex, but little dilated medially.

Remarks. The genitalia of the holotype are in poor condition, so its description and the illustration were extracted from the original publication. The illustration of the hypopygium is that of a paratype with same data of holotype, except for the date 28.III.2001 (UFES).

\section{Pseudisobrachium tridens Evans, 1969 Figs 386-389}

Holotype, male. ARGENT[INA], Tucumán, Horco Molle, San X\{J\}axier Mts., 15-21.V.1966, L. Stange col. (FIML).

Descriptions. Male. Length $4.7 \mathrm{~mm}$. Black. Mandible with five apical teeth, uppermost broad, upper three smaller than sublower. Clypeus with trapezoidal median lobe, apical margin convex medially, median carina bifurcated apically. Antennal segment XI 2.0x as long as wide, pubescence subappressed, as long as half of diameter of basal flagellomeres, with some long suberect setae outstanding pubescence. Eye long-haired. Frons strongly coriaceous, punctures dense and deep. WH 0.93x LH, WF 0.7x WH, WF 1.64x HE, DAO 0.14x WF, OOL 1.3x WOT, posterior ocellus distant from vertex crest 2.0x DAO. Vertex convex, corner rounded. VOL 1.26x HE. Temple parallel anteriorly. Notaulus short anteriorly. Propodeal disc $1.16 \mathrm{x}$ as wide as long, $0.79 \mathrm{x}$ as high as long, basal triangle defined, disc strigulate, median carina complete, posterior carina indistinguishable. Mesopleuron with nearly polished callus. Fore wing with discoidal vein weakly pigmented basally, not interstitial with median vein. Genitalia: paramere with ventral arm $2.5 \mathrm{x}$ wider than dorsal; aedeagus mesoconvex, its apex reaching beyond apex of dorsal arm of paramere.

\section{Pseudisobrachium tucumanum Evans, 1969}

Figs 390-394

Holotype, male. ARGENTINA, Tucumán, Horco Molle, San X\{J\}axier Mts., 4-12.I.1966, Malaise trap, L. Stange col. (FIML).

Descriptions. Male. Length $3.5 \mathrm{~mm}$. Dark castaneous. Mandible with five apical teeth, upper three teeth smaller than sublower. Clypeus with trapezoidal median lobe, apical margin concave, median carina bifurcated apically. Antennal segment XI 1.8x as long as wide, pubescence subappressed, longer than half of diameter of basal flagellomeres, with some long erect setae outstanding pubescence. Eye long-haired. Frons coriaceous, with shallow and large punctures. WH 1.07x LH, WF 0.69x WH, WF 1.54x HE, DAO 0.17x WF, OOL 1.36x WOT, posterior ocellus distant from vertex crest $0.68 \mathrm{x}$ DAO. Vertex straight, corner rounded. VOL 1.0x HE. Temple subparallel anteriorly. Notaulus nearly complete. Propodeal disc $1.1 \mathrm{x}$ as wide as long, $0.78 \mathrm{x}$ as high as long, basal triangle small, median carina incomplete, posterior carina ill defined. Mesopleuron with nearly polished callus. Fore wing with discoidal vein tubular on base, not interstitial with median vein. Metasoma with sting and female valvae.

Remarks. This male type presents female genitalia. I have never seen this fact in any other male in Bethylidae. The description of genitalia ahead is based on the only one paratype from the type locality. Genitalia: paramere with ventral arm $1.8 \mathrm{x}$ wider than dorsal; aedeagus mesoconvex, its apex not reaching apex of dorsal arm of paramere.

\section{Pseudisobrachium turbinatum Evans, 1961} Figs 395-398

Holotype, male. JAMAICA, 4000 feet, Hardwar Gap, 13.VII.1966, Howden \& Becker col. (CNCI, \#10993).

Descriptions. Male. Length $3.0 \mathrm{~mm}$. Dark castaneous. Mandible with five apical teeth, upper four small and nearly of equal size. Clypeus with trapezoidal median lobe, apical margin angularly concave. Antennal segment XI about 2.5 as long as wide, pubescence of basal flagellomeres long and suberect, with some longer erect setae outstanding pubescence. Eye bulging. Frons coriaceous, punctures inconspicuous. WH 0.93x LH, WF 0.63x WH, WF 1.2x HE, DAO 0.24x WF, OOL 1.0x WOT, posterior ocellus distant from vertex crest $0.75 \mathrm{x}$ DAO. Vertex narrow, nearly straight. VOL $0.85 x$ HE. Temple strongly divergent anterad. Pronotal disc depressed forward. Notaulus present on anterior half of mesoscutum. Propodeal disc $0.75 \mathrm{x}$ as wide as long, $0.5 \mathrm{x}$ as high as long, median carina incomplete, posterior carina absent. Mesopleuron with polished callus. Fore wing with discoidal vein pigmented, not interstitial with median vein. Genitalia: paramere with ventral arm $2.5 \mathrm{x}$ wider than dorsal arm; aedeagus mesoconvex, its apex aligned with apex of dorsal arm of paramere.

Remarks. This species is very similar to other Jamaican species $P$. pseudoobscurum, $P$. praecipum and $P$. howdeni by having the head very narrow posteriorly (Figs 395, 330, 326, 193, respectively). However $P$. pseudoobscurum has the apical margin of the median clypeal lobe straight (Fig. 330), versus angularly concave in P. turbinatum (Fig. 395); P. praecipum has the median clypeal lobe (Fig. 326), versus narrow in $P$. turbinatum (Fig. 395); P. howdeni has the eyes small (Fig. 193, versus large in P. turbinatum (Fig. 395).

\section{Pseudisobrachium uruguayense Ogloblin, 1938 Figs 399-402}

Holotype, male. URUGUAY (MACN, \#47734). Mounted in slides \#P99 and P100.

Descriptions. Male. Length $5.38 \mathrm{~mm}$. Black. Mandible with five apical teeth, upper four small and nearly of equal size. Clypeus with trapezoidal median lobe, apical margin barely convex, median carina incomplete. Antennal segment XI 1.57x 

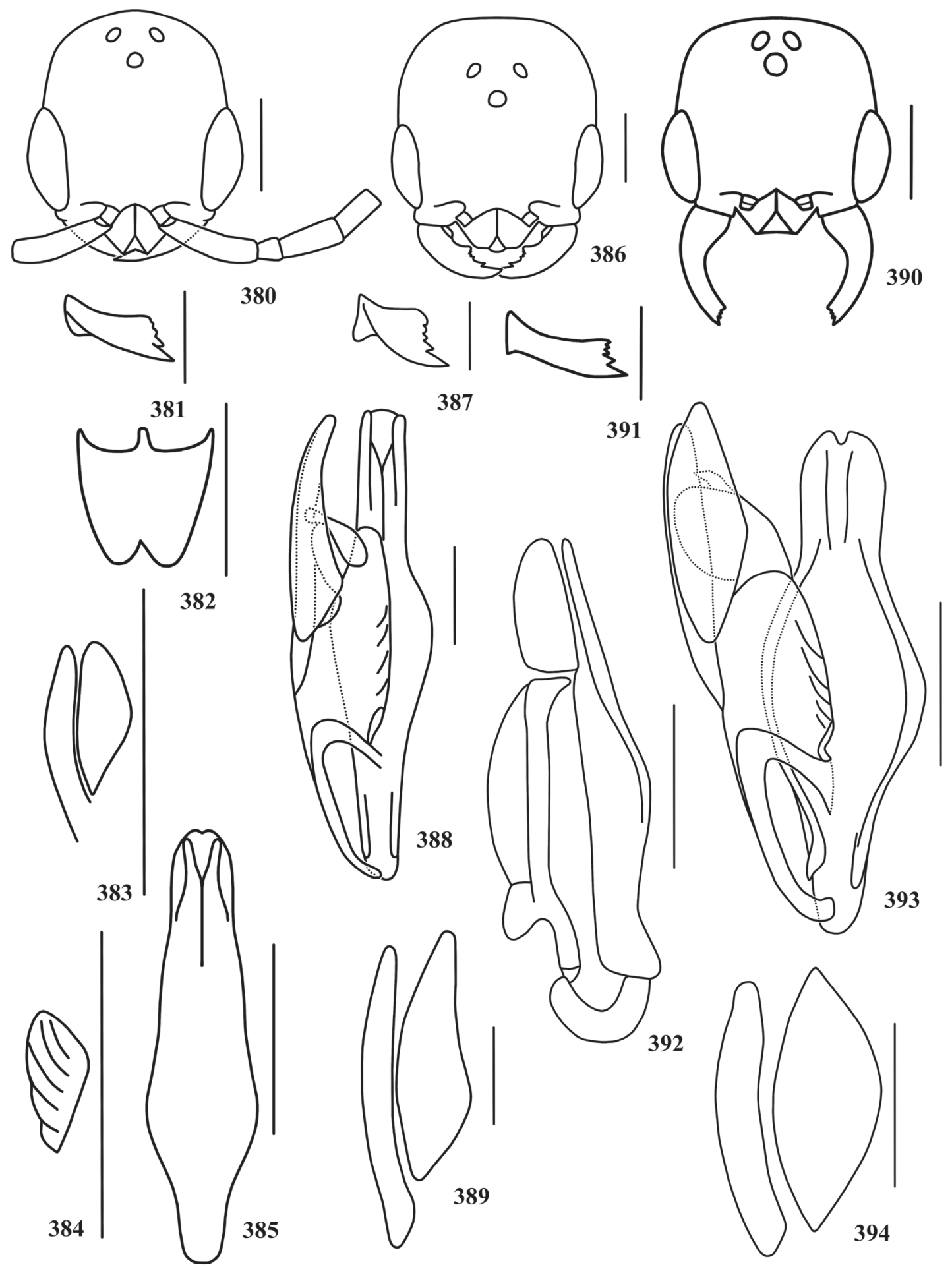

Figures 380-394. Pseudisobrachium triacutum holotype male: (380) head, dorsal; (381) mandible, frontal; (382) hypopygium, ventral; (383) paramere, full; (384) vannus, lateral; (385) aedeagus, ventral; P. tridens holotype male: (386) head, dorsal; (387) mandible, frontal; (388) genitalia, ventral; (389) paramere, full; P. tucumanum holotype male: (390) head, dorsal; (391) mandible, frontal; (392) genitalia, lateral; paratype: (393) genitalia, ventral; (394) paramere, full. Bar $=250 \mu \mathrm{m}$. 
as long as wide, pubescence subappressed, shorter than half of diameter of basal flagellomeres, with some long erect setae outstanding pubescence. Eye sparsely hairy. Frons strongly coriaceous, with shallow and large punctures. WH 1.02x LH, WF 0.62x WH, WF 1.21x HE, DAO 0.28x WF, OOL 0.37x WOT, posterior ocellus distant from vertex crest $0.68 \mathrm{x}$ DAO, posterior margin of anterior ocellus nearly reaching eye top imaginary line. Vertex slightly convex, corner rounded. VOL $0.63 x$ HE. Temple subparallel anteriorly. Notaulus short as anterior stub. Parapsidal furrow complete. Propodeal disc $0.81 \mathrm{x}$ as wide as long, $0.31 \mathrm{x}$ as high as long, basal triangle inconspicuous, median carina incomplete, posterior carina undefined, nearly absent. Mesopleuron with nearly polished callus. Prosternum with long spine at posterior margin. Fore wing with discoidal vein unpigmented, not interstitial with median vein. Genitalia: paramere with ventral arm 3.0x wider than dorsal; aedeagus mesoconvex, its apex slightly not reaching apex of dorsal arm of paramere.

Remarks. The holotype was clarified and prepared in slides. I was not able to examine precisely many characters because it is in bad conditions, so I decided to study the genitalia of a paratype which are in good condition. The genitalia are in separated slide, but curiously there are two genitalia in one preparation. Luckily they are essentially similar to each other.

\section{Pseudisobrachium ventriosum Waichert \& Azevedo, 2004 Figs 403-406}

Holotype, male. BRAZIL, E[spírito] S[anto], S[anta] Teresa, Est[ação] Biol[ógica de] Santa Lúcia, 23.IV.2001, sweeping, C.O. Azevedo \& R. Kawada col. (UFES).

Descriptions. Male. Length $5.8 \mathrm{~mm}$. Black; fore wing subhyaline. Mandible with four apical teeth, upper three teeth smaller than sublower. Clypeus with trapezoidal median lobe, apical margin concave, median carina bifurcated apicad, corner sharpened. Antennal segment XI 2.0x as long as wide; pubescence of basal flagellomeres subappressed, with some erect setae outstanding pubescence. Frons polished, punctures deep and conspicuous. WH 0.94x LH, WF 0.63x WH, WF 1.21x HE; DAO 0.15x WF; OOL 1.09x WOT, posterior ocellus distant from vertex crest $1.43 \mathrm{x}$ DAO, anterior margin of anterior ocellus nearly reaching eye top imaginary line. Vertex straight. VOL $0.64 x$ HE. Temple divergent anterad. Notaulus present on anterior third of mesoscutum. Propodeal disc $1.05 \mathrm{x}$ as wide as long, $0.72 \mathrm{x}$ as high as long, entirely rugulose, median carina incomplete, posterior carina absent; declivity rugulose, lateral of propodeum mostly polished. Mesopleuron with large and polished callus. Fore wing with discoidal vein weakly pigmented, interstitial with median vein. Genitalia: paramere with ventral arm more than $2.5 \mathrm{x}$ wider than dorsal arm, dorsal arm with subapical callus; aedeagus with sides slightly concave, its apex not reaching apex of dorsal arm of paramere.

Remarks. The genitalia are in poor condition.

\section{Pseudisobrachium werneri Evans, 1967 Figs 407-410}

Holotype, male. MEX[ICO], Son[ora], 10 mi E Navajoa, 13.VIII.1959, black light trap, W. L. Nutting \& F. G. Werner col. (MCZH).

Descriptions. Male. Length $3.6 \mathrm{~mm}$. Dark castaneous; fore wing weakly subhyaline. Mandible with five apical teeth, uppermost wide, upper four small and nearly of equal size. Clypeus with trapezoidal median lobe. Antenna with segment XI about $1.5 \mathrm{x}$ as long as wide, pubescence of basal flagellomeres appressed, with some long erect setae outstanding pubescence. Eye bulging, long-haired. Frons coriaceous, punctures shallow. WH 1.08x LH; WF 0.55x WH, WF 1.05x HE, ocelli large, DAO $0.29 \mathrm{x}$ WF, OOL $0.47 \mathrm{x}$ WOT, frontal angle of ocellar triangle compact slightly less than right, posterior ocellus distant from vertex crest $0.5 \mathrm{x} D A O$, anterior margin of anterior ocellus reaching eye top imaginary line. Vertex nearly straight, corner rounded. VOL $0.54 \mathrm{x}$ HE. Notaulus present on anterior third of mesoscutum. Propodeal disc $1.04 \mathrm{x}$ as wide as long, $0.76 \mathrm{x}$ as high as long, median carina incomplete, basal triangle small, disc otherwise polished and weakly coriaceous, posterior carina absent; declivity and lateral of propodeum aciculate. Mesopleuron with weakly coriaceous callus. Fore wing with discoidal vein unpigmented, interstitial with median vein. Genitalia: paramere with ventral arm 2.6x wider than dorsal; aedeagus narrow, little dilated, base and apex wide, its apex not reaching apex of dorsal arm of paramere, with additional pair of membranous lobes.

\section{Pseudisobrachium zeteki Evans, 1961 Figs $411-412$}

Holotype, female. [PANAMA], C[anal] Z[one], Barro Colo[rado] I[sland], VII.1941, lot \#41-20631, J. Zetek col. \#4852 (USNM, \#65157).

Descriptions. Female. Length $3.28 \mathrm{~mm}$. Dark castaneous. Mandible with four nearly of equal size apical teeth. Clypeus with median lobe trapezoidal, apical margin with few bristles, angularly concave in dorsal view, triangular in frontal view, median carina high and angled in profile. Eye light, subcircular, with one facet. Malar space as long as eye length. Frons polished, not so densely punctuated. Head with anterior half of sides straight and nearly parallel, vertex nearly straight, occipital carina visible in dorsal view, $1.28 \mathrm{x}$ as long as wide. Pronotal disc, mesoscutum and propodeal disc in ratio of 17:16:17, with deep and wide suture separating pronotal disc and mesoscutum. Mid tibia strongly spinose. Metasoma with short petiole.

\section{ACKNOWLEDGMENTS}

I am most grateful to all curators cited in the text for the loan of the material examined; to John Huber, Lubomir Masner, Henry Goulet, Gary Gibson and Jennifer Read for the reception during my two visits to CNCI, to Stephan Cover and Philip Perkins 


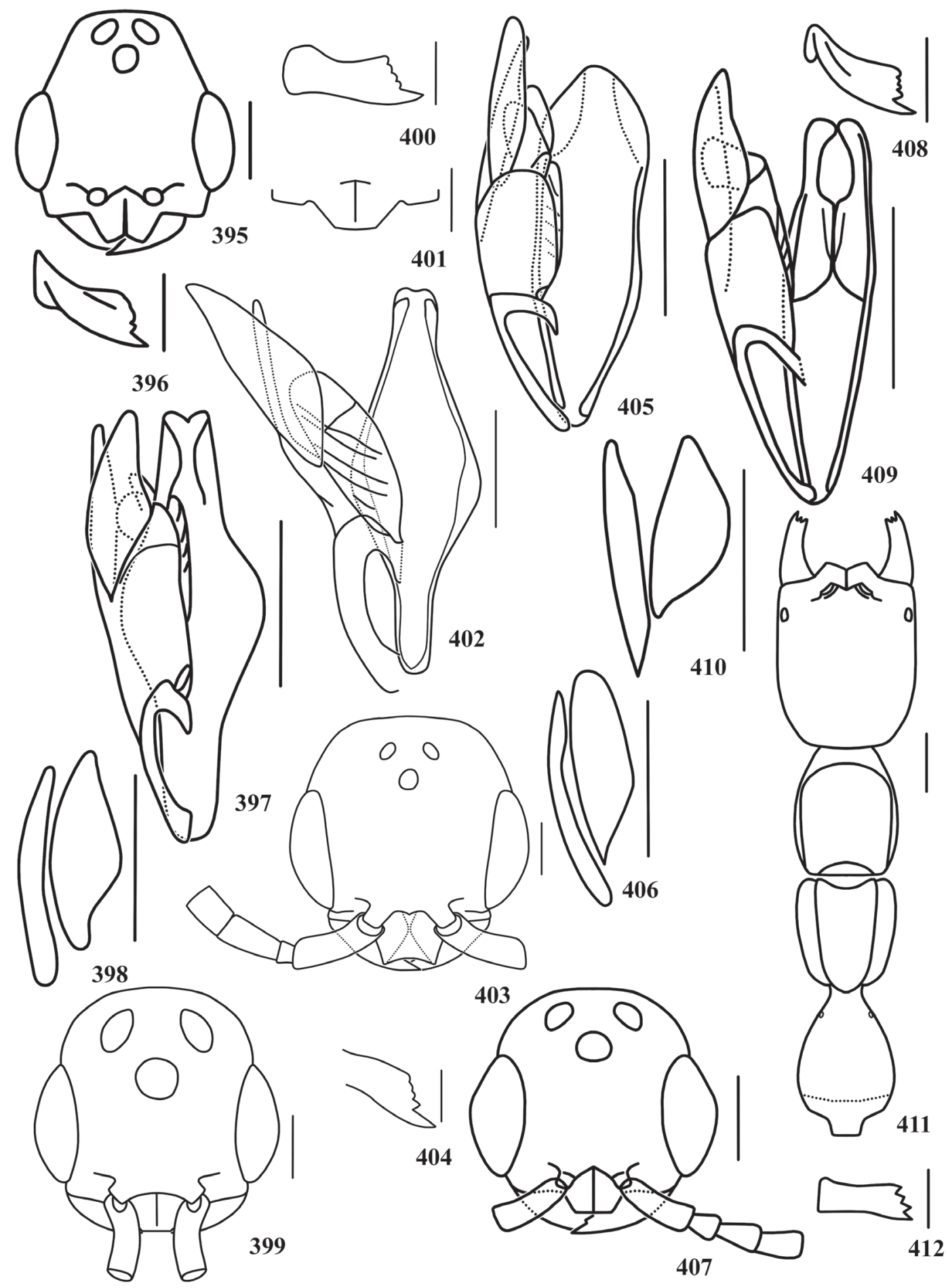

Figures 395-412. Pseudisobrachium turbinatum holotype male: (395) head, dorsal; (396) mandible, frontal; (397) genitalia, ventral; (398) paramere, full; P. uruguayense paratype male: (399) head, dorsal; (400) mandible, frontal; (401) clypeus, dorsal; (402) genitalia, ventral; P. ventriosum holotype male: (403) head, dorsal; (404) mandible, frontal; (405) genitalia, ventral; (406) paramere, full; P. werneri holotype male: (407) head, dorsal; (408) mandible, frontal; (409) genitalia, ventral; (410) paramere, full; P. zeteki holotype female: (411) head and mesosoma, dorsal; (412) mandible, frontal. Bar $=250 \mu \mathrm{m}$. 
for the reception during my visit to $\mathrm{MCZH}$, Kim Goodger and George Else for the reception during my visit to BMNH; to Ted Schultz, David Furth, Eugenie Okonsky and Brian Harris for the reception during my two visits to USNM; to Virginia Colomo and Alexandra Soria for the reception during my visit to FIML; to Arturo Roig Alsina for the reception during my passage to MACN; to Roberto Poggi for the reception during my passage to MCSN; to Monica Ospina, Diana Aires and Juan Manuel Vargas Rojas for the reception during my visit to IAVH; Norman F. Johnson and Luciana Musetti for offering their laboratory facilities at The Ohio State University where I revised material from many collections; to following people for hosting me very kindly in their home: Alexandra Soria and her family especially her mother Nelida Argentina de la Cruz during my visit to FIML; Gilmar Perin during my visit to MCZH; Diana Aires and her family while in Bogotá, going for and back to IAVH; Monik Oprea and Daniel Brito during my second visit to USNM; Doreen Watlers during my two visits to $\mathrm{CNCI}$; to Monica Ospina in name of IAVH for providing me free hotel accommodation while visiting IAVH; to Grisel Cabrera Dávila for transporting types from and to ACC; to Ariel Camousseight for providing information on Chilean collections; to Paulo R. W. Stein who edited the plates; to Fernanda T. Gobbi for providing many suggestions of characters analysis and key; to Alexandre P. Aguiar for cleaning the English of some parts of this text; Marcelo T. Tavares who informed me on type material of Museo de la Plata. The author benefited from The Ernest Mayr Grant - U.S.A., which provided financial support to the trips to Boston (year grant 2004) and London (year grant 2006), to CanaColl grant \#194/2002 by financial support of my first trip to Ottawa, and from a fellowship and financial support from FAPES - Brazil (grant \#32837330/2006) and CNPq - Brazil (grants \#500280/00-8, \#303216/2004-2 and \#306331/2007-7).

\section{LITERATURE CITED}

Ashmead, W.H. 1893. A monograph of the North American Proctotrypidae. Bulletin of the United States National Museum 45: 1-463.

AsHMEAD, W.H. 1894. Report on the parasitic Cynipidae, part of the Braconidae, the Ichneumonidae, the Proctotrypidae, and part of the Chalcididae. Part III. [Hymenoptera of the Island of St. Vincent]. Journal of the Linnean Society of London, Zoology, 25: 188-254.

Azevedo, C.O. 1991. Comentário dos gêneros de Bethylidae (Hymenoptera, Aculeata) da região de São Carlos, SP, Brasil. Anais do VI Seminário Regional de Ecologia: 483-496.

Azevedo, C.O. 1996. Sobre os Bethylidae (Hymenoptera, Chrysidoidea) da Reserva Indígena de Tadarimana, Rondonópolis, MT, Brasil. Anais do VII Seminário Regional de Ecologia: 111-119.

Azevedo, C.O. \& J.L. Helmer. 1999. Ecologia de comunidade de Bethylidae (Hymenoptera, Chrysidoidea) da Reserva Biológica do Roncador, Brasília, DF, Brasil. Revista Brasileira de Zoologia 16: 1115-1126.
Azevedo, C.O.; J.L. Helmer; F. Barreto. 2006. Análise da fauna de Bethylidae (Hymemnoptera, Chrysidoidea) de oito localidades do Paraná, Brasil. Boletim do Museu de Biologia Mello Leitão, Nova Série, 19: 83-94.

Azevedo, C.O.; J.L. Helmer \& E. Morato. 2002. Diversidade de vespas Bethylidae (Insecta, Hymenoptera) do Parque Nacional da Serra do Divisor, Acre, Brasil e seu uso no plano de manejo e conservação da área. Acta Amazônica 32: 71-81. BRuch, C. 1917a. Insectos mirmecófilos. Physis 3: 141-49.

BRuch, C. 1917b. Nuevas capturas de insectos mirmecófilos. Physis 3: 458-465.

Cameron, P. 1888. Insecta. Hymenoptera (Families Tenthredinidae - Chrysididae). Subfamily Bethylinae. Biologia CentraliAmerica 1: 448-457.

Cameron, P. 1909. A contribution to the knowledge of the parasitic Hymenoptera of Argentina. Transactions of the American Entomological Society 35: 419-450.

Evans, H.E. 1961. A revision of the genus Pseudisobrachium in the North and Central America (Hymenoptera, Bethylidae). Bulletin of the Museum of Comparative Zoology 126: 211-318.

Evans, H.E. 1964. A synopsis of the American Bethylidae (Hymenoptera, Aculeata). Bulletin of the Museum of Comparative Zoology 132: 1-222.

Evans, H.E. 1966. Further studies on Neotropical Pristocerinae (Hymenoptera, Bethylidae). Acta Hymenopterologica 2: 99-117.

Evans, H.E. 1967. Notes on Mexican and southwestern U.S. Bethylidae (Hymenoptera): Part I, Pristocerinae. Entomological News 78: 13-23.

Evans, H.E. 1969a. The genus Pseudisobrachium in Argentina and Chile (Hymenoptera, Bethylidae). Acta Zoologica Lilloana 25: 43-66.

Evans, H.E. 1969b. West Indian wasps of the subfamily Pristocerinae (Hymenoptera, Bethylidae). Proceedings of the Entomological Society of Washington 71: 514-530.

Evans, H.E. 1973. Further studies on South American Bethylidae (Hymenoptera). Proceedings of the Entomological Society of Washington 75: 194-204.

Evans, H.E. 1978. The Bethylidae of America North of Mexico. Memoirs of the American Entomological Institute 27: 1332.

FouTs, R.M. 1928. Notes on the Bethylinae with descriptions of one new Cuban and twelve new North American species (Hym.). Proceedings of the Entomological Society of Washington 30: 121-32.

Gordh, G. \& L. Moczar. 1990. A catalog of the world Bethylidae (Hymenoptera). Memoirs of the American Entomological Institute 46: 1-364.

Harris, R .A. 1979. A glossary of surface sculpturing. Occasional Papers in Entomology 28: 1-31.

Kieffer, J.J. 1904. Description de nouveaux Dryininae et Bethylinae. Du Musée Civique de Genes. Annali del Museo 
Civico di Storia Naturale di Genova, Serie 3, 1 (41): 351412.

KiefFer, J.J. 1906 (1905). Beschreibung neuer Proctotrypiden aus Nord und Zentralamerika. Berliner Entomologische Zeitschrift 50: 237-90.

KIEFFER, J.J. 1910a. Description de nouveaux microhyménoptères du Brésil. Annales de la Société Entomologique de France 78: 287-348.

Kieffer, J.J. 1910b. Description de nouveaux béthylides (Hyménoptères). Annales de la Société Entomologique de France 79: 31-56.

ManN, W.M. 1915. Some myrmecophilous insects from Hayti. Psyche 22: 161-66.

Ogloblin, A.A. 1925a. Description of a new species of Pseudoisobrachium Kieff. (Hym. Bethylidae) from Brazil. Casopis Ceskoslovenske Spolecnosti Entomologicke 20: 24-7. [1924]

Ogloblin, A.A. 1925b. Descriptions of three new species of Pseudoisobrachium Kieff. (Hym. Bethylidae) from Brazil. Casopis Ceskoslovenske Spolecnosti Entomologicke 21: 77-81. [1924]

Ogloblin, A.A. 1938. Descripciones de Bethylidae y Drynidae de las colecciones del Museo Argentino de Ciencias Natu- rales. Anales del Museo Argentino de Ciencias Naturales 40: 35-50.

Ogloblin, A.A. 1950. Dos "Bethyloidea" nuevos de la colección de la Fundación Miguel Lillo (Hymenopt.). Acta Zoologica Lilloana 9: 487-93.

Ogloblin, A.A. 1963. Especies nuevas del género Pseudoisobrachium Kieff. (Fam. Bethylidae, Hymenoptera). Revista de la Sociedad Entomologica Argentina 26: 133-38.

Perez, D’A.V. 1981 (1980). El género Pseudisobrachium Kieffer, en Chile (Hymenoptera: Bethylidae: Pristocerinae). Revista Chilena de Entomologia 10: 61-2.

Richards, O. W. 1977. Hymenoptera. Introduction and key to families. London, Royal Entomological Society of London, $2^{\text {nd }}$ ed., 100p.

Vargas R., J.M. \& M. Terayama. 2002. Five new species of the subfamily Pristocerinae (Insecta, Hymenoptera, Bethylidae) from Colombia. Biogeography 4: 25-31

Waichert, C. \& C.O. Azevedo. 2004. Fourteen new species of Pseudisobrachium (Hymenoptera, Bethylidae) from Atlantic rain forest of Espírito Santo, Brazil. Zootaxa 661: 1-22.

Westwood, J.O. 1874. Thesaurus Entomologicus Oxoniensis. Oxford, Clarendon Press, vol. 4, 205p.

Received: 05.VI.2008; Accepted: 03.XII.2008.

Editorial responsibility: José Albertino Rafael 